LU TP 11-07

February 2011

\title{
Meson-meson Scattering in QCD-like Theories
}

\author{
Johan Bijnens and Jie Lu \\ Department of Astronomy and Theoretical Physics, Lund University, \\ Sölvegatan 14A, SE 223-62 Lund, Sweden
}

\begin{abstract}
We discuss meson-meson scattering at next-to-next-to-leading order in the chiral expansion for QCD-like theories with general $n$ degenerate flavours for the cases with a complex, real and pseudo-real representation. I.e. with global symmetry and breaking pattern $S U(n)_{L} \times S U(n)_{R} \rightarrow S U(n)_{V}, S U(2 n) \rightarrow$ $S O(2 n)$ and $S U(2 n) \rightarrow S p(2 n)$. We obtain fully analytical expressions for all these cases. We discuss the general structure of the amplitude and the structure of the possible intermediate channels for all three cases. We derive the expressions for the lowest partial wave scattering length in each channel and present some representative numerical results. We also show various relations between the different cases in the limit of large $n$.
\end{abstract}

PACS: 


\section{Introduction}

In an earlier paper [1] we started the phenomenology of QCD-like theories at nextto-next-to-leading (NNLO) order in the light mass expansion in their respective lowenergy effective theories. The motivation for this work is that these theories are interesting as variations on QCD and could play some role as models for a nonperturbative Higgs sector. Early work in this context are the technicolor variations of [2, 3, 4]. Recent reviews of more modern developments are [5, 6]. Lattice calculations have started to explore these type of theories as well, some references are [7]. The main interest in these theories is in the massless limit but lattice simulations are necessarily performed at a finite fermion mass. In [1] we worked out a number of simple observables, the mass, decay constant and vacuum-expectation-value to NNLO in these theories. Here we work out the amplitude for meson-meson scattering to the same order. In lattice calculations the amplitude for meson-meson scattering is not directly accessible but the scattering lengths can be derived from the dependence on the volume of the lattice [8]. We therefore also provide explicit expressions for the scattering lengths.

The EFT relevant for dynamical electroweak symmetry breaking can have different patterns of spontaneous breaking of the global symmetry than QCD. The resulting Goldstone Bosons, or pseudo-Goldstone bosons in the presence of mass terms, are thus in different manifolds and the low-energy EFT is also different.

In this paper we only discuss the same cases as in [1] where the underlying strong interaction is vectorlike and all fermions have the same mass. Three main patterns of global symmetry show up. A thorough discussion tree level or lowest order (LO) is [9]. With $n$ fermions 1 in a complex representation the global symmetry group is $S U(n)_{L} \times S U(n)_{R}$ and it is expected to be spontaneously broken to the diagonal subgroup $S U(n)_{V}$. This is the direct extension of the QCD case. For $n$ fermions in a real representation the global symmetry group is $S U(2 n)$ and it is expected to be spontaneously broken to $S O(2 n)$. In the case of two colours and $n$ fermions in the fundamental (pseudo-real) representation the global symmetry group is again $S U(2 n)$ but here it is expected to be spontaneously broken to an $S p(2 n)$ subgroup. Earlier references are [10, 11, 12]. Some earlier work for the complex case and the pseudo-real case at NLO can be found in [13, 14, 15].

In the remainder of this paper we refer to the complex representation case as complex or QCD, the real representation case as adjoint or real and the pseudo-real representation case as two-colour or pseudo-real. In [1] we extended the construction of the general Lagrangian to NLO2 including the divergence structure. The NNLO for the QCD case is in [16] and the divergence structure in [17]. The Lagrangian constructed in [16] is with the changes discussed in [1] and in Sect. 2 also a complete Lagrangian for the other two cases but we have not shown it to be minimal nor calculated the divergence structure.

We do not repeat the discussion of the three different cases at the underlying fermion (quark) level. This can be found in [9] and [1, Sect. 2. In Sect. 2 we quote the structure of the effective field theories for the three cases but we again refer to [1]

\footnotetext{
${ }^{1}$ We use $n$ rather than $N_{F}$ for the number of flavours since it makes the formulas shorter.

${ }^{2}$ References to some related work can be found in [5].
} 
for more details. Sect. 3 discusses in detail the general structure of the amplitude. The amplitude can be expressed in terms of two functions $B(s, t, u)$ and $C(s, t, u)$ which are generalizations of the amplitude $A(s, t, u)$ in $\pi \pi$-scattering [18]. We work out the possible intermediate states using the relevant group theory and using a projection operator formalism obtain the amplitudes in the different channels. The results for the amplitude are discussed in Sect. 4 and for the scattering lengths in Sect. 5. Here we present some representative numerical results for the scattering lengths as well as some large $n$ relations between the different cases. The lengthier formulas at two-loop order are given in an appendix. This work needed a few more integrals at intermediate stages than [19, 20, these are given in App. D. In Sect. 6] we summarize our results.

\section{Effective Field Theory}

\subsection{Generators}

The notation for the three cases can be brought in a very similar form. More details can be found in [1]. The Goldstone boson live on a manifold $G / H$ where $G$ is the full global symmetry group and $H$ is the part that remains unbroken after spontaneous symmetry-breaking. We label the unbroken generators as $T^{a}$ and the broken ones as $X^{a}$.

The space $S U(n) \times S U(n) / S U(n)$ is isomorphic to $S U(n)$ so we use the $X^{a}$ as the generators of $S U(n)$ for the QCD case. They are traceless, hermitian $n \times n$ matrices.

The adjoint or real case has the generators in $S U(2 n) / S O(2 n)$ where the broken generators satisfy

$$
J_{S} X^{a}=\left(X^{a}\right)^{T} J_{S}, \quad \text { with } \quad J_{S}=\left(\begin{array}{cc}
0 & I \\
I & 0
\end{array}\right) .
$$

$I$ is the $n \times n$ unit matrix and the superscript $T$ indicates the transpose. The $X^{a}$ are traceless, hermitian $2 n \times 2 n$ matrices in this case. Multiplying (11) with $J_{S}$ from left and right leads immediately to

$$
X^{a} J_{S}=J_{S}\left(X^{a}\right)^{T} .
$$

The two-colour or pseudo-real case has the generators in $S U(2 n) / S p(2 n)$ where the broken generators satisfy

$$
J_{A} X^{a}=\left(X^{a}\right)^{T} J_{A}, \quad \text { with } \quad J_{A}=\left(\begin{array}{cc}
0 & -I \\
I & 0
\end{array}\right) .
$$

The $X^{a}$ are traceless, hermitian $2 n \times 2 n$ matrices also in this case. Multiplying (3) with $J_{A}$ similar to above gives

$$
X^{a} J_{A}=J_{A}\left(X^{a}\right)^{T} .
$$

The unbroken generators satisfy

$$
\begin{aligned}
S O(2 n): & T^{a} J_{S}+J_{S} T^{a T}=0 \\
S p(2 n): & T^{a} J_{S}+J_{S} T^{a T}=0 .
\end{aligned}
$$


This allows in both cases to derive using $J=J_{S}$ or $J=J_{A}$ respectively:

$$
h^{\dagger} J=J h^{T} \quad \text { with } \quad h=\exp i h^{a} T^{2} .
$$

We always use generators normalized to one:

$$
\left\langle T^{a} T^{b}\right\rangle=\left\langle X^{a} X^{b}\right\rangle=\delta^{a b} .
$$

$\langle A\rangle=\operatorname{tr}_{F}(A)$, is the trace over the flavour indices. This is over $n$ for the QCD case and $2 n$ for the real and pseudo-real case.

During the course of the calculation, we often have to sum over the Goldstone Bosons. These sums can be easily performed using

complex :

$$
\begin{aligned}
\left\langle X^{a} A X^{a} B\right\rangle & =\langle A\rangle\langle B\rangle-\frac{1}{n}\langle A B\rangle, \\
\left\langle X^{a} A\right\rangle\left\langle X^{a} B\right\rangle & =\langle A B\rangle-\frac{1}{n}\langle A\rangle\langle B\rangle .
\end{aligned}
$$

Real :

$$
\begin{aligned}
\left\langle X^{a} A X^{a} B\right\rangle & =\frac{1}{2}\langle A\rangle\langle B\rangle+\frac{1}{2}\left\langle A J_{S} B^{T} J_{S}\right\rangle-\frac{1}{2 n}\langle A B\rangle, \\
\left\langle X^{a} A\right\rangle\left\langle X^{a} B\right\rangle & =\frac{1}{2}\langle A B\rangle+\frac{1}{2}\left\langle A J_{S} B^{T} J_{S}\right\rangle-\frac{1}{2 n}\langle A\rangle\langle B\rangle .
\end{aligned}
$$

Pseudoreal :

$$
\begin{aligned}
\left\langle X^{a} A X^{a} B\right\rangle & =\frac{1}{2}\langle A\rangle\langle B\rangle+\frac{1}{2}\left\langle A J_{A} B^{T} J_{A}\right\rangle-\frac{1}{2 n}\langle A B\rangle, \\
\left\langle X^{a} A\right\rangle\left\langle X^{a} B\right\rangle & =\frac{1}{2}\langle A B\rangle-\frac{1}{2}\left\langle A J_{A} B^{T} J_{A}\right\rangle-\frac{1}{2 n}\langle A\rangle\langle B\rangle .
\end{aligned}
$$

There is a relation that the broken generators satisfy for the real and pseudo-real case.

$$
\left\langle X^{a} X^{b} \ldots X^{k} X^{l}\right\rangle=\left\langle X^{l} X^{k} \ldots X^{b} X^{a}\right\rangle .
$$

The proof for the real case is

$$
\begin{aligned}
\left\langle X^{a} X^{b} \ldots X^{k} X^{l}\right\rangle & =\left\langle X^{a} X^{b} \ldots X^{k} X^{l} J_{S}^{2}\right\rangle \\
& =\left\langle X^{a} X^{b} \ldots X^{k} J_{S} X^{l T} J_{S}\right\rangle \\
& =\left\langle X^{a} X^{b} \ldots J_{S} X^{k T} X^{l T} J_{S}\right\rangle \\
& =\left\langle J_{S} X^{a T} X^{b T} \ldots X^{k T} X^{l T} J_{S}\right\rangle \\
& =\left\langle X^{a T} X^{b T} \ldots X^{k T} X^{l T}\right\rangle \\
& =\left\langle\left(X^{l} X^{k} \ldots X^{b} X^{a}\right)^{T}\right\rangle \\
& =\left\langle X^{l} X^{k} \ldots X^{b} X^{a}\right\rangle
\end{aligned}
$$

The pseudo-real case is proven by replacing $J_{S}^{2}$ by $-J_{A}^{2}$ and following the same steps. (9) is also the reason why the Lagrangian in [16] is not minimal for the real and pseudo-real case.

In the group theory references there is a conjecture mentioned that to get from $S O(2 n)$ to $S p(2 n)$ it is sufficient to take $n \rightarrow-n$. This feature is indeed visible in most of our formulas. 


\subsection{Lagrangians}

As described in more detail in [1] we can write the Lagrangians in the three cases in a very similar way. The Goldstone Boson manifold $G / H$ is parametrized by

$$
u=\exp \left(\frac{i}{\sqrt{2} F} \phi\right), \quad \phi=\phi^{a} X^{a} .
$$

These transform under the symmetry transformation in the QCD case for $g_{L} \times$ $g_{R} \in S U(n)_{L} \times S U(n)_{R}$ as

$$
u \rightarrow g_{R} u h\left(g_{L}, g_{R}, \phi\right)^{\dagger}=h\left(g_{L}, g_{R}, \phi\right) u g_{L}^{\dagger} .
$$

$h$ is the socalled compensator field and is defined by (12) and is also an $S U(n)$ matrix. This can be derived from the standard general formulation [21] as done in [1]. For a transformation in the conserved part of the group we have that $g_{L}=g_{R}=g_{V}$ and $h=g_{V}$.

The notation for the other two cases is directly that of [21]. A symmetry transformation $g \in G=S U(2 n)$ transforms $u$ as

$$
u \rightarrow g u h(g, \phi)^{\dagger}, \quad \text { with } \quad h=\exp \left(i h^{a} T^{a}\right) .
$$

I.e. $h$ is in the unbroken part $H$ of the group. In case the transformation $g$ is in the conserved part of the group, $g \in H$, we have that $h=g$.

We can now define the quantities

$$
\begin{aligned}
u_{\mu} & =i\left(u^{\dagger} \partial_{\mu} u-u \partial_{\mu} u^{\dagger}\right), \\
\Gamma_{\mu} & =\frac{1}{2}\left(u^{\dagger} \partial_{\mu} u-u \partial_{\mu} u^{\dagger}\right) .
\end{aligned}
$$

Under the group transformation in all cases we have $u_{\mu} \rightarrow h u_{\mu} h^{\dagger}$ and $\Gamma_{\mu}$ can be used to define a covariant derivative.

$$
\nabla_{\mu} u_{\nu} \equiv \partial_{\mu} u_{\nu}+\Gamma_{\mu} u_{\nu}-u_{\nu} \Gamma_{\mu} \rightarrow h \nabla_{\mu} u_{\nu} h^{\dagger}
$$

In [1] we also showed how the external fields can be included in a similar way as for the QCD case in [22, 13]. In particular the quark masses can be put in a quantity $\chi_{ \pm}$that transforms as $\chi_{ \pm} \rightarrow h \chi_{ \pm} h^{\dagger}$.

The lowest order Lagrangian takes on the standard form

$$
\mathcal{L}_{L O}=\frac{F^{2}}{4}\left\langle u_{\mu} u^{\mu}+\chi_{+}\right\rangle
$$

for all three cases and the same is true for the NLO Lagrangian.

$$
\begin{aligned}
\mathcal{L}_{N L O}= & L_{0}\left\langle u^{\mu} u^{\nu} u_{\mu} u_{\nu}\right\rangle+L_{1}\left\langle u^{\mu} u_{\mu}\right\rangle\left\langle u^{\nu} u_{\nu}\right\rangle+L_{2}\left\langle u^{\mu} u^{\nu}\right\rangle\left\langle u_{\mu} u_{\nu}\right\rangle \\
& +L_{3}\left\langle u^{\mu} u_{\mu} u^{\nu} u_{\nu}\right\rangle+L_{4}\left\langle u^{\mu} u_{\mu}\right\rangle\left\langle\chi_{+}\right\rangle+L_{5}\left\langle u^{\mu} u_{\mu} \chi_{+}\right\rangle+L_{6}\left\langle\chi_{+}\right\rangle^{2} \\
& +L_{7}\left\langle\chi_{-}\right\rangle^{2}+\frac{1}{2} L_{8}\left\langle\chi_{+}^{2}+\chi_{-}^{2}\right\rangle .
\end{aligned}
$$

We have kept only the terms contributing to meson-meson scattering in (17).

The NNLO Lagrangian is known for the complex or QCD case [16] as well as its divergence structure [17]. The same Lagrangian with the changes mentioned above is complete for the other two cases but probably not minimal. We have nonetheless chosen to leave the contributions from those terms in the results quoted here. 


\subsection{Renormalization}

We use the standard renormalization procedure in ChPT [22, 13] with the extension to NNLO described in great detail in [20, 17]. The divergences at NLO are canceled by the subtractions as calculated in [1]. At NNLO the divergences for the QCD case are canceled by the subtractions calculated in [17]. The other two cases satisfy all the expected constraints. Nonlocal divergences fully cancel, the $\epsilon$ parts of the loop integrals as defined in App. D always cancel and the double divergences satisfy the Weinberg relations [17].

As usual in ChPT we apply the $\overline{\mathrm{MS}}$ scheme of dimensional regularization, in which the bare LECs $L_{i}$ are defined as

$$
L_{i}=(c \mu)^{d-4}\left[\Gamma_{i} \Lambda+L_{i}^{r}(\mu)\right]
$$

Where the dimension $d=4-2 \epsilon$, and

$$
\begin{aligned}
\Lambda & =\frac{1}{16 \pi^{2}(d-4)}, \\
\ln c & =-\frac{1}{2}\left[\ln 4 \pi+\Gamma^{\prime}(1)+1\right] .
\end{aligned}
$$

The coefficients $\Gamma_{i}$ can be found in [22, 1] for the complex and in [1] for the real and pseudo-real $S p(2 n)$ case.

The NNLO terms can be made finite with the subtractions

$$
K_{i}=(c \mu)^{2(d-4)}\left[K_{i}^{r}-\Gamma_{i}^{(2)} \Lambda^{2}-\left(\frac{1}{16 \pi^{2}} \Gamma_{i}^{(1)}+\Gamma_{i}^{(L)}\right) \Lambda\right]
$$

The coefficients $\Gamma_{i}^{(2)}, \Gamma_{i}^{(1)}$ and $\Gamma_{i}^{(L)}$ for the complex case have been derived in [17]. For the real and pseudo-real case, the results do not exist. We have checked that all remaining divergences are local and can thus be subtracted.

\section{General results for the amplitudes}

\section{$3.1 \pi \pi$ case}

The $\pi \pi$ scattering amplitude, which correspond to the QCD case with $n=2$ is well known. Due to crossing and the possible $S U(2)$ (isospin) invariants the amplitude can be written as [18, 23]

$$
M_{\pi \pi}(s, t, u)=\delta^{a b} \delta^{c d} A(s, t, u)+\delta^{a c} \delta^{b d} A(t, u, s)+\delta^{a d} \delta^{b c} A(u, s, t) .
$$

The function $A(s, t, u)$ is symmetric under the interchange of $t$ and $u$.

The possible states of two pions are isospin 0,1 or 2 . The amplitude for the three channels are given by 23 ]

$$
\begin{aligned}
& T^{0}(s, t, u)=3 A(s, t, u)+A(t, s, u)+A(u, t, s), \\
& T^{1}(s, t, u)=A(t, s, u)-A(u, s, t), \\
& T^{2}(s, t, u)=A(t, s, u)+A(u, s, t) .
\end{aligned}
$$


Where $I$ is isospin, and $P_{I}$ is the projection operator on isospin $I$ They satisfy the relation

$$
M_{\pi \pi}(s, t, u)=\sum_{I=0,2} T^{I}(s, t, u) P_{I}
$$

and

$$
T^{I}(s, t, u) P_{I}(\text { no sum })=P_{I} M_{\pi \pi}(s, t, u) .
$$

In the remainder of this section we will generalize these results. (22) is generalized in terms of two functions in Sect. 3.2. The possible intermediate states and the corresponding amplitudes are derived for the three cases separately in the last three subsections of this section.

\subsection{General amplitude}

The amplitude for meson-meson scattering is given by

$$
\left\langle\phi^{c}\left(p_{c}\right) \phi^{d}\left(p_{d}\right) \mid \phi^{a}\left(p_{a}\right) \phi^{b}\left(p_{d}\right)\right\rangle=M(s, t, u) .
$$

The Mandelstam variables $s, t, u$ are defined by

$$
s=\left(p_{a}+p_{b}\right)^{2} / M_{\mathrm{phys}}^{2}, \quad t=\left(p_{a}-p_{c}\right)^{2} / M_{\mathrm{phys}}^{2}, \quad u=\left(p_{a}-p_{d}\right)^{2} / M_{\mathrm{phys}}^{2} .
$$

These satisfy

$$
s+t+u=4 \text {. }
$$

We have chosen here to use the dimensionless versions in order to simplify later formulas.

The flavour structure of the amplitude for meson-meson scattering can be described by constructing all possible invariants from the four corresponding generator matrices $X^{e}, e=a, b, c, d$. Taking into account that $\left\langle X^{e}\right\rangle=0$ there are 9 invariants possible

$$
\begin{array}{rll}
\left\langle X^{a} X^{b} X^{c} X^{d}\right\rangle, & \left\langle X^{a} X^{c} X^{d} X^{b}\right\rangle, & \left\langle X^{a} X^{d} X^{b} X^{c}\right\rangle, \\
\left\langle X^{a} X^{d} X^{c} X^{b}\right\rangle, & \left\langle X^{a} X^{b} X^{d} X^{c}\right\rangle, & \left\langle X^{a} X^{c} X^{b} X^{d}\right\rangle, \\
\left\langle X^{a} X^{b}\right\rangle\left\langle X^{c} X^{d}\right\rangle, & \left\langle X^{a} X^{c}\right\rangle\left\langle X^{b} X^{d}\right\rangle, & \left\langle X^{a} X^{d}\right\rangle\left\langle X^{b} X^{c}\right\rangle .
\end{array}
$$

Under charge conjugation $X^{a} \longrightarrow X^{a T}$. This means that the amplitudes multiplying the first row in (29) must be the same as those multiplying the second row 3 As a result the full amplitude can be written in terms of two invariant amplitudes $B(s, t, u)$ and $C(s, t, u)$.

$$
\begin{aligned}
M(s, t, u)= & {\left[\left\langle X^{a} X^{b} X^{c} X^{d}\right\rangle+\left\langle X^{a} X^{d} X^{c} X^{b}\right\rangle\right] B(s, t, u) } \\
& +\left[\left\langle X^{a} X^{c} X^{d} X^{b}\right\rangle+\left\langle X^{a} X^{b} X^{d} X^{c}\right\rangle\right] B(t, u, s) \\
& +\left[\left\langle X^{a} X^{d} X^{b} X^{c}\right\rangle+\left\langle X^{a} X^{c} X^{b} X^{d}\right\rangle\right] B(u, s, t) \\
& +\delta^{a b} \delta^{c d} C(s, t, u)+\delta^{a c} \delta^{b d} C(t, u, s)+\delta^{a d} \delta^{b c} C(u, s, t) .
\end{aligned}
$$

\footnotetext{
${ }^{3}$ Alternatively use (9) for the real and pseudo-real case.
} 
The flavour structure also implies that

$$
B(s, t, u)=B(u, t, s) \quad C(s, t, u)=C(s, u, t) .
$$

For $n=3$ there is the Cayley-Hamilton relation

$$
\sum_{6 \text { permutations }}\left\langle X^{a} X^{b} X^{c} X^{d}\right\rangle=\sum_{3 \text { permutations }}\left\langle X^{a} X^{b}\right\rangle\left\langle X^{c} X^{d}\right\rangle,
$$

which allows for an ambiguity in the split of $B$ and $C$. For $n=2$ we can perform all the traces with four matrices $X^{a}$ in terms of Kronecker deltas.

The relation between the general amplitudes and the $\pi \pi$ scattering case (22) is

$$
A(s, t, u)=C(s, t, u)+B(s, t, u)+B(t, u, s)-B(u, s, t) .
$$

Note that the property (31) insures that $A(s, t, u)$ is symmetric under the interchange of $t$ and $u$ as it should be. The form (22) holds also for any set of pions. I.e., taking any $S U(2)$ subgroup of the unbroken group and any three of the pseudo Goldstone bosons that form a triplet under such a group, one can rewrite the general amplitude (30) into (22) using (33).

\subsection{QCD case: channels and amplitudes}

The Goldstone boson transform under the conserved part of the group, $S U(n)$, as

$$
\phi \rightarrow h \phi h^{\dagger}
$$

This means that they are in the adjoint representation of $S U(n)$. For the $n=2,3$ case we have an isospin triplet under $S U(2)$ and an octet under $S U(3)$. The intermediate states for these are well known:

$$
\begin{aligned}
& S U(2): \quad 3 \otimes 3=1 \oplus 3 \oplus 5(\text { or } I=0,1,2), \\
& S U(3): \quad 8 \otimes 8=1 \oplus 8_{S} \oplus 8_{A} \oplus 10 \oplus \overline{10} \oplus 27 .
\end{aligned}
$$

The group theory for $S U(n)$ can be done in many ways. One is via Young diagrams and the second using tensor methods. We will do both. The $S U(n)$ case derived here was in fact known [24] and our results are in agreement with his. Young diagrams for $S U(n)$ are explained in [25] page 370. The Young diagrams for $S U(n)$ give

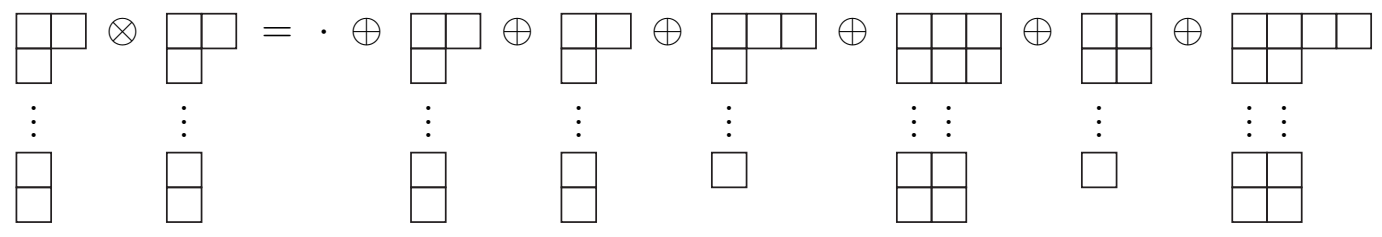

Note that the $\vdots$ stand for $n-5$ boxes. In terms of free indices the right hand side of (36) is no indices (singlet), twice one upper and one lower index (adjoint). The remaining four have all two lower and two upper indices, where the upper indices are produced from the columns with length $n-1$ and $n-2$ boxes using the Levi-Civita tensor $\epsilon^{i_{1} \ldots i_{n}}$. For these we use the notation $R_{X}^{Y}$ where $X=S, A$ indicate whether 
the lower indices are symmetric or antisymmetric and $Y=S, A$ the same for the upper indices. The decomposition (36) can thus be written as

$$
A d j . \otimes A d j .=R_{I} \oplus R_{S} \oplus R_{A} \oplus+R_{S}^{A} \oplus R_{A}^{S} \oplus R_{A}^{A} \oplus R_{S}^{S}
$$

The order of the irreducible representation on the right-hand side is the same in (36) and (37).

If we have a particular two-meson state $\phi^{a}\left(p_{1}\right) \phi^{b}\left(p_{2}\right)$ we have to write it in terms of states that belong to the irreducible multiplets to obtain the amplitudes for the different channels. We use a simplified notation below with $A=X^{a}, B=X^{b}, C=X^{c}$ and $D=X^{d}$ for many of the terms. All traces connecting a lower with an upper index must vanish.

- $R_{I}$ : singlet representation. All indices should be contracted, so this must be proportional to $A_{i}^{j} B_{j}^{i}=\langle A B\rangle$. Summing over the $n^{2}-1$ states that are present tells us that the correct normalized state is

$$
R_{I}=\frac{1}{\sqrt{n^{2}-1}} \sum_{a, b}\left\langle X^{a} X^{b}\right\rangle \phi^{a} \phi^{b}
$$

A projection operator $P_{I}^{a b c d}$ that projects on the singlet component is

$$
P_{I}^{a b c d}=\frac{1}{n^{2}-1}\langle A B\rangle\langle C D\rangle .
$$

It can be checked that this is a projection operator

$$
P_{I}^{a b c d} P_{I}^{c d e f}=P_{I}^{a b e f}
$$

using (8) and $\sum_{a, b} \delta_{a b}=n^{2}-1$.

- $R_{S}$ : adjoint symmetric representation. This needs in the end an upper and a lower index and must be traceless. We choose here to split up the two possible contractions of the indices of $A$ and $B$ in way that is symmetric under the interchange of $A$ and $B$.

$$
\left(R_{S}\right)_{j}^{i}=\sqrt{\frac{n}{2\left(n^{2}-4\right)}}\left[A_{j}^{m} B_{m}^{i}+B_{j}^{m} A_{m}^{i}-\frac{2}{n} \delta_{j}^{i}\langle A B\rangle\right] .
$$

The last term is needed to make $R_{S}$ traceless. The normalization can be worked out by checking the normalization of a particular state or by checking that the projection operator 4

$$
P_{S}=R_{S}(A, B)_{j}^{i} R_{S}(C, D)_{i}^{j}
$$

has the correct normalization, its square is equal to itself. This leads finally to the projection operator

$$
P_{S}=\frac{n}{2\left(n^{2}-4\right)}\left[\langle(A B+C D)(C D+D C)\rangle-\frac{4}{n}\langle A B\rangle\langle C D\rangle\right] .
$$

Suppressing the indices we get similar to (40) $P_{S}^{2}=P_{S}$ and $P_{S} P_{I}=P_{I} P_{S}=0$.

\footnotetext{
${ }^{4}$ We suppress the superscript abcd from now on.
} 
- $R_{A}$ : adjoint anti-symmetric representation

$$
\left(R_{A}\right)_{j}^{i}=\frac{1}{\sqrt{2 n}}\left(A_{j}^{m} B_{m}^{i}-B_{j}^{m} A_{m}^{i}\right) .
$$

$R_{A}(A, B)$ is traceless and antisymmetric in $A$ and $B$. The projection operator corresponding to this is

$$
P_{A}=\frac{-1}{2 n}\langle(A B-B A)(C D-D C)\rangle .
$$

- $R_{S}^{A}$ : symmetric for lower indices and antisymmetric for upper. A state here corresponds to

$$
\begin{aligned}
\left(R_{S}^{A}\right)_{k l}^{i j}= & \frac{1}{2}\left[A_{k}^{i} B_{l}^{j}+\frac{1}{n} \delta_{k}^{i}\left(A_{m}^{j} B_{l}^{m}-A_{l}^{m} B_{m}^{j}\right)\right] \\
& -(i \leftrightarrow j)+(k \leftrightarrow l)-(i \leftrightarrow j, k \leftrightarrow l) .
\end{aligned}
$$

The projection operator on this type of states is

$$
\begin{aligned}
P_{S A}= & \left(R_{S}^{A}(A, B)\right)_{k l}^{i j}\left(R_{S}^{A}(C, D)\right)_{i j}^{k l} \\
= & \frac{1}{4 n}[\langle(A B-B A)(C D-D C)\rangle+n(\langle A C B D\rangle \\
& -\langle A D B C\rangle)+n(\langle A C\rangle\langle B D\rangle-\langle A D\rangle\langle C D\rangle)] .
\end{aligned}
$$

- $R_{A}^{S}$ : antisymmetric for lower indices and symmetric for upper. A state here corresponds to

$$
\begin{aligned}
\left(R_{A}^{S}\right)_{k l}^{i j}= & \frac{1}{2}\left[A_{k}^{i} B_{l}^{j}-\frac{1}{n} \delta_{k}^{i}\left(A_{m}^{j} B_{l}^{m}-A_{l}^{m} B_{m}^{j}\right)\right] \\
& +(i \leftrightarrow j)-(k \leftrightarrow l)-(i \leftrightarrow j, k \leftrightarrow l) .
\end{aligned}
$$

The projection operator on this type of states is

$$
\begin{aligned}
P_{A S}= & \frac{1}{4 n}[\langle(A B-B A)(C D-D C)\rangle-n(\langle A C B D\rangle \\
& -\langle A D B C\rangle)+n(\langle A C\rangle\langle B D\rangle-\langle A D\rangle\langle C D\rangle)] .
\end{aligned}
$$

- $R_{S}^{S}$ : symmetric for both upper index and lower index. The states are

$$
\begin{aligned}
\left(R_{S}^{S}\right)_{k l}^{i j}= & \frac{1}{2}\left[A_{k}^{i} B_{l}^{j}-\frac{1}{n+2} \delta_{k}^{i}\left(A_{l}^{m} B_{m}^{j}+B_{l}^{m} A_{m}^{j}\right)\right. \\
& \left.+\frac{1}{(n+1)(n+2)} \delta_{k}^{i} \delta_{l}^{j}\langle A B\rangle\right] \\
& +(i \leftrightarrow j)+(k \leftrightarrow l)+(i \leftrightarrow j, k \leftrightarrow l)
\end{aligned}
$$

and the projection operator is

$$
\begin{aligned}
P_{S S}= & \frac{-1}{4(n+2)}\langle(A B+B A)(C D+D C)\rangle+\frac{1}{4}(\langle A C B D\rangle \\
& +\langle A D B C\rangle)+\frac{1}{4}(\langle A C\rangle\langle B D\rangle+\langle A D\rangle\langle C D\rangle) \\
& +\frac{1}{2(n+1)(n+2)}\langle A B\rangle\langle C D\rangle .
\end{aligned}
$$


- $R_{A}^{A}$ : antisymmetric for both upper index and lower index. The states are

$$
\begin{aligned}
\left(R_{A}^{A}\right)_{k l}^{i j}= & \frac{1}{2}\left[A_{k}^{i} B_{l}^{j}+\frac{1}{n-2} \delta_{k}^{i}\left(A_{l}^{m} B_{m}^{j}+B_{l}^{m} A_{m}^{j}\right)\right. \\
& \left.-\frac{1}{(n-1)(n-2)} \delta_{k}^{i} \delta_{l}^{j}\langle A B\rangle\right] \\
& -(i \leftrightarrow j)-(k \leftrightarrow l)+(i \leftrightarrow j, k \leftrightarrow l)
\end{aligned}
$$

and the projection operator is

$$
\begin{aligned}
P_{A A}= & \frac{-1}{4(n-2)}\langle(A B+B A)(C D+D C)\rangle-\frac{1}{4}(\langle A C B D\rangle \\
& +\langle A D B C\rangle)+\frac{1}{4}(\langle A C\rangle\langle B D\rangle+\langle A D\rangle\langle C D\rangle) \\
& +\frac{1}{2(n-1)(n-2)}\langle A B\rangle\langle C D\rangle .
\end{aligned}
$$

The projection operators agree with those of [24] and using (9) can be shown to satisfy $P_{r} P_{r^{\prime}}=P_{r} \delta_{r r^{\prime}}$ for $r$ the various representations. One last check is that

$$
\sum_{r} P_{r}=\langle A C\rangle\langle B D\rangle \text {. }
$$

The right-hand side is the unit operator when acting on the product of two states in the adjoint representation. It can also be seen that $R_{I}, R_{S}, R_{A}^{A}$ and $R_{S}^{S}$ are symmetric under interchanging $A$ and $B$ while $R_{A}, R_{A}^{S}$ and $R_{S}^{A}$ are antisymmetric.

The amplitude in the different intermediate states can now be extracted from the general amplitude in two equivalent ways. We can pick a state $R_{r}$ in a representation $r$ and get it via

$$
T_{r}=\left\langle R_{r}|M(s, t, u)| R_{r}\right\rangle
$$

or apply the projection operators on the full amplitude with

$$
P_{r} T_{r}=P_{r} M(s, t, u) .
$$

Both methods give as expected the same result but the second one is much easier to apply. For the first method it is best to choose a state where the terms with $\delta$ functions are not present. E.g. for the four index representations take a state $R_{r}$ with $i=1, j=2, k=3, l=4$. For evaluating (56) one can use (86).

$$
\begin{aligned}
T_{I}= & 2\left(n-\frac{1}{n}\right)[B(s, t, u)+B(t, u, s)]-\frac{2}{n} B(u, s, t) \\
& +\left(n^{2}-1\right) C(s, t, u)+C(t, u, s)+C(u, s, t), \\
T_{S}= & \left(n-\frac{4}{n}\right)[B(s, t, u)+B(t, u, s)]-\frac{4}{n} B(u, s, t) \\
& +C(t, u, s)+C(u, s, t), \\
T_{A}= & n[-B(s, t, u)+B(t, u, s)]+C(t, u, s)-C(u, s, t), \\
T_{S A}= & C(t, u, s)-C(u, s, t), \\
T_{A S}= & C(t, u, s)-C(u, s, t), \\
T_{S S}= & 2 B(u, s, t)+C(t, u, s)+C(u, s, t), \\
T_{A A}= & -2 B(u, s, t)+C(t, u, s)+C(u, s, t) .
\end{aligned}
$$


They satisfy the relation similar to (24),

$$
M(s, t, u)=\sum_{r} T_{r}(s, t, u) P_{r}
$$

One also notices that $T_{S A}=T_{A S}$ in general from (57).

\subsection{Real case: channels and amplitudes}

In this subsection we work out the possible two meson intermediate states for the case of $S U(2 n) / S O(2 n)$. One problem is that the mesons transform under $S O(2 n)$ as $\phi \rightarrow h \phi h^{\dagger}$. The matrices $h \in S O(2 n)$ in the embedding introduced here do not simply satisfy $h h^{T}=1$ either. In our case the $S O(2 n)$ is instead defined as $h J_{S} h^{T}=J_{S}$. The easiest way to obtain objects that appear in the usual way is to note that using ([6)

$$
\phi J_{S} \rightarrow h \phi h^{\dagger} J_{S}=h \phi J_{S} h^{T}
$$

and that an invariant trace on these object needs an extra factor of $J_{S}$. E.g.

$$
\left(\phi^{a} J_{S}\right) J_{S}\left(\phi^{b} J_{S}\right) \rightarrow h\left(\phi^{a} J_{S}\right) h^{T} J_{S} h\left(\phi^{b} J_{S}\right) h^{T}=h\left(\phi^{a} J_{S}\right) J_{S}\left(\phi^{b} J_{S}\right) h^{T}
$$

Keeping that in mind we can use the standard way of dealing with $S O(2 n)$. Note that $\left(\phi J_{S}\right)^{T}=J_{S} \phi^{T}=\phi J_{S}$ so the Goldstone bosons live in the symmetric representation of $S O(2 n)$.

The method of Young tableaux has been generalized to $S O(2 n)$ [26]. Putting together two symmetric representations gives

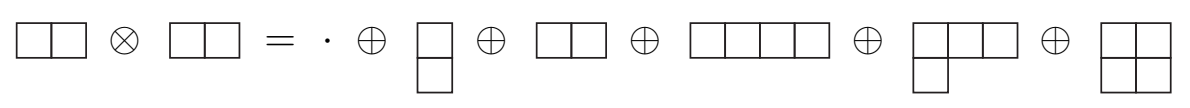

We can write this in the form

$$
\text { Sym. } \otimes \text { Sym. }=R_{I} \oplus R_{A} \oplus R_{S} \oplus R_{F S} \oplus R_{M A} \oplus R_{M S} .
$$

The states are given in Tab. 1, They are made traceless but remember that indices of $\phi^{a} J_{S}$ and $\phi^{b} J_{S}$ are always contracted with $J_{S}$.

As an example the singlet representation $R_{I}$ is proportional to

$$
\left(\phi^{a} J_{S}\right)_{i j} J_{S i k} J_{S j l}\left(\phi^{b} J_{S}\right) k l=\left\langle\phi^{a} \phi^{b}\right\rangle \text {. }
$$

The two-index antisymmetric representation $R_{A}$ is, now using $A, B$ for $\phi^{a}, \phi^{b}$,

$$
\left(A J_{S}\right)_{i k}\left(B J_{S}\right)_{j l} J_{S k l}-\left(A J_{S}\right)_{j k}\left(B J_{S}\right)_{i l} J_{S k l}=(A B J-B A J)_{i j}
$$

where we heavily used $J_{S}^{2}=1$ and the fact that $A J_{S}$ and $B J_{S}$ are symmetric matrices. One can also easily check that the trace $\left(A B J_{S}-B A J_{S}\right)_{i j} J_{S i j}$ vanishes.

Then $R_{A}$ and $R_{S}$ are antisymmetric respectively symmetric both under $i \leftrightarrow j$ and $A \leftrightarrow B$. The remaining ones are the four-index representations. $R_{F S}$ is fully symmetric in all indices and under $A \leftrightarrow B$. The two remaining representations have a mixed symmetry in the indices but are antisymmetric respectively symmetric under $A \leftrightarrow B$. 


\begin{tabular}{|c|c|}
\hline$R_{I}$ & $\frac{1}{\sqrt{(2 n-1)(n+1)}}\langle A B\rangle$ \\
\hline$R_{A}$ & $(A B J-B A J)_{i j}$ \\
\hline$R_{S}$ & $(A B J+B A J)_{i j}-\frac{1}{n} J_{i j}\langle A B\rangle$ \\
\hline$R_{F S}$ & $(A J)_{i j}(B J)_{k l}-\frac{1}{n+2}\left[J_{i j}(A B J+B A J)_{k l}\right]+\frac{1}{2(n+2)(n+1)} J_{i j} J_{k l}\langle A B\rangle$ \\
& $+(i j k l \leftrightarrow i k j l)+(i j k l \leftrightarrow i l j k)+(i j k l \leftrightarrow k l i j)$ \\
& $+(i j k l \leftrightarrow j l i k)+(i j k l \leftrightarrow j k i l)$ \\
\hline$R_{M A}$ & $(A J)_{i j}(B J)_{k l}-(A J)_{k l}(B J)_{i j}-\frac{1}{2 n+2}\left[J_{i k}(A B J-B A J)_{j l}\right.$ \\
& $\left.+J_{j k}(A B J-B A J)_{i l}+J_{i l}(A B J-B A J)_{j k}+J_{j l}(A B J-B A J)_{i k}\right]$ \\
\hline$R_{M S}$ & $(A J)_{i j}(B J)_{k l}+(A J)_{k l}(B J)_{i j}-(A J)_{i k}(B J)_{j l}-(A J)_{j l}(B J)_{i k}$ \\
& $+\frac{1}{2(n-1)}\left[J_{i j}(A B J+B A J)_{k l}+J_{k l}(A B J+B A J)_{i j}\right.$ \\
& $\left.-J_{i k}(A B J+B A J)_{j l}-J_{j l}(A B J+B A J)_{i k}\right]$ \\
& $-\frac{1}{(n-1)(2 n-1)}\left(J_{i j} J_{k l}-J_{i k} J_{j l}\right)\langle A B\rangle$ \\
\hline
\end{tabular}

Table 1: The intermediate states for the real or adjoint case, $S U(2 n) / S O(2 n)$. The notations $A, B$ stands for $\phi^{a}$ and $\phi^{b}$ and $J$ is $J_{S}$ everywhere.

\begin{tabular}{|c|c|}
\hline$P_{I}$ & $\frac{1}{(2 n-1)(n+1)}\langle A B\rangle\langle C D\rangle$ \\
\hline$P_{A}$ & $-\frac{1}{2(n+1)}\langle(A B-B A)(C D-D C)\rangle$ \\
\hline$P_{S}$ & $\frac{n}{2(n-1)(n+2)}\left(\langle(A B+B A)(C D+D C)\rangle-\frac{2}{n}\langle A B\rangle\langle C D\rangle\right)$ \\
\hline$P_{F S}$ & $\frac{1}{6} \frac{2}{(n+1)(n+2)}\langle A B\rangle\langle C D\rangle+\langle A C\rangle\langle B D\rangle+\langle A D\rangle\langle B C\rangle$ \\
& $\left.+2\langle A C B D+A D B C\rangle-\frac{2}{n+2}\langle(A B+B A)(C D+D C)\rangle\right]$ \\
\hline$P_{M A}$ & $\frac{1}{2(n+1)}\langle(A B-B A)(C D-D C)\rangle+\frac{1}{2}(\langle A C\rangle\langle B D\rangle-\langle A D\rangle\langle B C\rangle)$ \\
\hline$P_{M S}$ & $\frac{1}{6}\left[\frac{2}{(n-1)(2 n-1)}\langle A B\rangle\langle C D\rangle+2(\langle A C\rangle\langle B D\rangle+\langle A D\rangle\langle B C\rangle)\right.$ \\
& $\left.-2\langle A D B C+A C B D\rangle-\frac{1}{n-1}\langle(A B+B A)(C D+D C)\rangle\right]$ \\
\hline
\end{tabular}

Table 2: The projection operator for the different intermediate states for the real or adjoint case, $S U(2 n) / S O(2 n)$. 
The corresponding projection operators are given in Tab. 2. These can be obtained by contracting the indices with $J_{S}$ of the states once with $A, B$ and once with $C, D$.

We can now use Tabs. 1 and 2 to project out the amplitudes in the various channels using (55) or (56). The results are

$$
\begin{aligned}
T_{I}= & \frac{1}{n}(2 n-1)(n+1)[B(s, t, u)+B(t, u, s)]+\frac{1}{n}(n-1) B(u, s, t) \\
& +(2 n-1)(n+1) C(s, t, u)+C(t, u, s)+C(u, s, t) \\
T_{A}= & -(1+n)[B(s, t, u)-B(t, u, s)]+C(t, u, s)-C(u, s, t) \\
T_{S}= & \frac{1}{n}(n-1)(n+2)[B(s, t, u)+B(t, u, s)]+\frac{1}{n}(n-2) B(u, s, t) \\
& +C(t, u, s)+C(u, s, t) \\
T_{F S}= & 2 B(u, s, t)+C(t, u, s)+C(u, s, t) \\
T_{M A}= & C(t, u, s)-C(u, s, t) \\
T_{M S}= & -B(u, s, t)+C(t, u, s)+C(u, s, t) .
\end{aligned}
$$

\subsection{Pseudo-real case: channels and amplitudes}

In this subsection we work out the possible two meson intermediate states for the case of $S U(2 n) / S p(2 n)$. One problem is that the mesons transform under $S p(2 n)$ as in $\phi \rightarrow h \phi h^{\dagger}$. The easiest way to obtain objects that appear in the usual way is to note that using (6)

$$
\phi J_{A} \rightarrow h \phi h^{\dagger} J_{A}=h \phi J_{A} h^{T}
$$

and that an invariant trace on these object needs an extra factor of $J_{A}$. Keeping that in mind we can use the standard way of dealing with $S p(2 n)$. Note that $\left(\phi J_{A}\right)^{T}=J_{A}^{T} \phi^{T}=-J_{A} \phi^{T}=\phi J_{S}$ so the Goldstone bosons live in the antisymmetric representation of $S p(2 n)$.

The method of Young tableaux also is developed for $S p(2 n)$ [27]. Putting together two antisymmetric representations gives

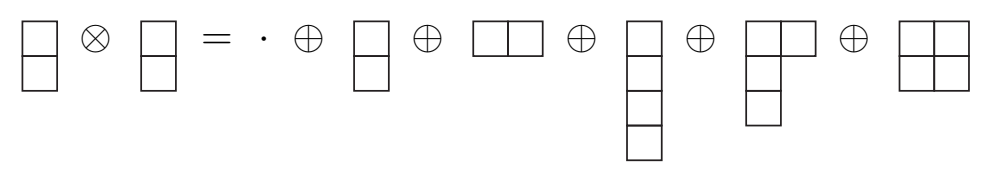

We can write this in the form

$$
\text { Asym. } \otimes \text { Asym. }=R_{I} \oplus R_{A} \oplus R_{S} \oplus R_{F A} \oplus R_{M A} \oplus R_{M S} .
$$

The states are given in Tab. 1. They are made traceless but remember that indices of $\phi^{a} J_{A}$ and $\phi^{b} J_{A}$ are always contracted with $J_{A}$.

The representations are the singlet representation, symmetric under $A \leftrightarrow B, R_{A}$ which is antisymmetric under the interchange $i \leftrightarrow j$ but symmetric under $A \leftrightarrow B$ and $R_{S}$ which is symmetric under the interchange $i \leftrightarrow j$ but antisymmetric under $A \leftrightarrow B$ Let us show the latter on $R_{A}$ The two-index antisymmetric representation 


\begin{tabular}{|c|c|}
\hline$R_{I}$ & $\frac{1}{\sqrt{(2 n+1)(n-1)}}\langle A B\rangle$ \\
\hline$R_{A}$ & $(A B J+B A J)_{i j}-\frac{1}{n} J_{i j}\langle A B\rangle$ \\
\hline$R_{S}$ & $(A B J-B A J)_{i j}$ \\
\hline$R_{F A}$ & $(A J)_{i j}(B J)_{k l}+\frac{1}{n-2} J_{i j}(A B J+B A J)_{k l}-\frac{1}{2(n-1)(n-2)} J_{i j} J_{k l}\langle A B\rangle$ \\
& $-(i j k l \leftrightarrow i k j l)+(i j k l \leftrightarrow i l j k)+(i j k l \leftrightarrow k l i j)$ \\
& $+(i j k l \leftrightarrow j l i k)+(i j k l \leftrightarrow j k i l)$ \\
\hline$R_{M A}$ & $(A J)_{i j}(B J)_{0} k l-(A J)_{k l}(B J)_{0} i j-\frac{1}{2 n-2}\left[J_{i k}(A B J-B A J)_{j l}\right.$ \\
& $\left.+J_{j l}(A B J-B A J)_{i k}-J_{i l}(A B J-B A J)_{j k}-J_{j k}(A B J-B A J)_{i l}\right]$ \\
\hline$R_{M S}$ & $\left\{(A J)_{i j}(B J)_{k l}+(A J)_{i k}(B J)_{j l}-\frac{1}{2(n+1)}\left[J_{i j}(A B J+B A J)_{k l}\right.\right.$ \\
& $\left.\left.-J_{i k}(A B J+B A J)_{j l}\right]+(i j \leftrightarrow k l)\right\}$ \\
& $+\frac{1}{2(n+1)(2 n-1)}\left(J_{i j} J_{k l}-J_{i k} J_{j l}\right)\langle A B\rangle$ \\
\hline
\end{tabular}

Table 3: The intermediate states for the pseudo-real or two-colour case, $S U(2 n) / S p(2 n)$. $J$ means $J_{A}$.

$R_{A}$ is, now using $A, B$ for $\phi^{a}, \phi^{b}$,

$$
\begin{aligned}
& \left(A J_{A}\right)_{i k}\left(B J_{A}\right)_{j l} J_{A k l}-\left(A J_{A}\right)_{j k}\left(B J_{A}\right)_{i l} J_{A k l} \\
& \quad=-\left(A J_{A}\right)_{i k} J_{A k l}\left(B J_{A}\right)_{l j} J_{A k l}-\left(B J_{A}\right)_{i l} J_{A l k}\left(A J_{A}\right)_{k i} \\
& =(A B J+B A J)_{i j}
\end{aligned}
$$

where we used $J_{A}^{2}=-1$ and the fact that $A J_{A}, B J_{A}$ and $J_{A}$ are antisymmetric matrices.

The remaining ones are the four-index representations. $R_{F A}$ is fully antisymmetric in all indices and symmetric under $A \leftrightarrow B$. The two remaining representations have a mixed symmetry in the indices but are antisymmetric respectively symmetric under $A \leftrightarrow B$. The states are give in Tab. 3 ,

The projection operators can be constructed by contracting all indices with $J_{A}$ of the states once with $A, B$ and once with $C, D$. The results are given in Tab. 4 .

We can now use again (55) or (56) to obtain the amplitudes in the different channels. The results are very similar to the real case and read

$$
\begin{aligned}
T_{I}= & \frac{1}{n}(2 n+1)(n-1)[B(s, t, u)+B(t, u, s)]-\frac{1}{n}(n+1) B(u, s, t) \\
& +(2 n+1)(n-1) C(s, t, u)+C(t, u, s)+C(u, s, t) \\
T_{A}= & \frac{1}{n}(n+1)(n-2)[B(s, t, u)+B(t, u, s)]-\frac{1}{n}(n+2) B(u, s, t) \\
& +C(t, u, s)+C(u, s, t) \\
T_{S}= & (1-n)[B(s, t, u)-B(t, u, s)]+C(t, u, s)-C(u, s, t) \\
T_{F A}= & -2 B(u, s, t)+C(t, u, s)+C(u, s, t) \\
T_{M A}= & C(t, u, s)-C(u, s, t) .
\end{aligned}
$$




\begin{tabular}{|c|c|}
\hline$P_{I}$ & $\frac{1}{(2 n+1)(n-1)}\langle A B\rangle\langle C D\rangle$ \\
\hline$P_{A}$ & $\frac{n}{2(n+1)(n-2)}\left(\langle(A B+B A)(C D+D C)\rangle-\frac{2}{n}\langle A B\rangle\langle C D\rangle\right)$ \\
\hline$P_{S}$ & $-\frac{1}{2(n-1)}\langle(A B-B A)(C D-D C)\rangle$ \\
\hline$P_{F A}$ & $\frac{1}{6}\left[\frac{2}{(n-1)(n-2)}\langle A B\rangle\langle C D\rangle+\langle A C\rangle\langle B D\rangle+\langle A D\rangle\langle B C\rangle\right.$ \\
& $\left.-2\langle A C B D+A D B C\rangle-\frac{2}{n-2}\langle(A B+B A)(C D+D C)\rangle\right]$ \\
\hline$P_{M A}$ & $\frac{1}{2(n-1)}\langle(A B-B A)(C D-D C)\rangle+\frac{1}{2}(\langle A C\rangle\langle B D\rangle-\langle A D\rangle\langle B C\rangle)$ \\
\hline$P_{M S}$ & $\frac{1}{3}\left[\frac{1}{(n+1)(2 n+1)}\langle A B\rangle\langle C D\rangle+\langle A C\rangle\langle B D\rangle+\langle A D\rangle\langle B C\rangle\right.$ \\
& $\left.+\langle A C B D+A D B C\rangle-\frac{1}{2(n+1)}\langle(A B+B A)(C D+D C)\rangle\right]$ \\
\hline
\end{tabular}

Table 4: The projection operator for the different channels for the pseudo-real or two-colour case, $S U(2 n) / S p(2 n)$.

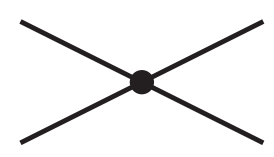

(1)

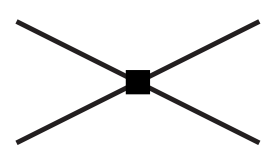

$(2)$

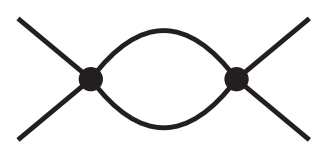

(3)

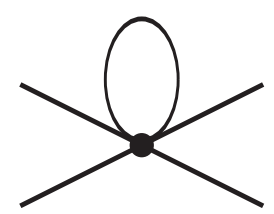

(4)

Figure 1: The leading order and next-to leading order for meson-meson scattering $\phi \phi \rightarrow \phi \phi$. The filled circle is a vertex from $\mathcal{L}_{2}$, and the filled square is a vertex from $\mathcal{L}_{4}$.

$$
T_{M S}=B(u, s, t)+C(t, u, s)+C(u, s, t),
$$

\section{Results for the amplitude $M(s, t, u)$}

We have rewritten the amplitudes here in terms of the physical decay constant $F_{\text {phys }}$ and mass $M_{\text {phys }}^{2}$. The relation of these to the lowest order results can be found in [1]. We also use the abbreviations

$$
x_{2}=\frac{M_{\text {phys }}^{2}}{F_{\text {phys }}^{2}}, \quad L=\frac{1}{16 \pi^{2}} \ln \frac{M_{\text {phys }}^{2}}{\mu^{2}}, \quad \pi_{16}=\frac{1}{16 \pi^{2}} .
$$

In addition we have often used (28) to simplify the expressions.

\subsection{Lowest order}

The lowest order result comes from the simple tree-level diagram (1) of Fig. 1. 


$$
B_{L O}(s, t, u)=x_{2}\left(-\frac{1}{2} t+1\right), \quad C_{L O}(s, t, u)=0,
$$

for all cases. This reproduces using the relation (33) Weinberg's result [18] for $\pi \pi$ scattering

$$
A_{L O}=x_{2}(s-1)
$$

\subsection{Next-to-leading order}

The next-to-leading order contains the 3 diagrams (2-4) in Fig. 1 in addition to wave-function-renormalization. The divergences from loop diagram (3) and (4) can be canceled by the bare low energy constants (LECs) of $\mathcal{L}_{4}$ in the diagram (2).

The functions $B(s, t, u)$ and $C(s, t, u)$ can be calculated from the one-loop graphs shown in Fig. 1(2-4) and wave-function renormalization. It also contains terms from rewriting the lowest-order result in the physical mass and decay constant.

The functions $B(s, t, u)$ and $C(s, t, u)$ can be rewritten in the form

$$
\begin{aligned}
& B(s, t, u)=x_{2}^{2}\left[B_{P}(s, t, u)+B_{S}(s, t-u)+B_{S}(u, t-s)+B_{T}(t)\right], \\
& C(s, t, u)=x_{2}^{2}\left[C_{P}(s, t, u)+C_{S}(s)+C_{T}(t)+C_{T}(u)\right] .
\end{aligned}
$$

$B_{P}(s, t, u)$ and $C_{P}(s, t, u)$ are the polynomial part, the remaining pieces are often called the unitarity correction. This can be proven using an extension of the methods of [28].

Using (28) we rewrite the polynomial part in its simplest form satisfying the symmetry constraints (31):

$$
\begin{aligned}
& B_{P}(s, t, u)=\alpha_{1}+\alpha_{2} t+\alpha_{3} t^{2}+\alpha_{4}(s-u)^{2}, \\
& C_{P}(s, t, u)=\beta_{1}+\beta_{2} s+\beta_{3} s^{2}+\beta_{4}(t-u)^{2} .
\end{aligned}
$$

The polynomial part for the three cases is give in Tab. 5.

The unitarity correction is given in Tab 6 . We noticed that the $C$ functions for the $S O(2 n)$ and $S p(2 n)$ case are the same.

\subsection{Next-to-next-to-leading order}

There are 13 diagrams at next-to-next-to-leading order shown in Fig. 2. We have checked that the nonlocal divergence cancels for all three cases and that for the complex or QCD case the result is fully finite with the subtractions calculated in [17.

The diagrams (15) and (16) are often called the sunset and vertex or fish diagram respectively. These require the most difficult integrals. At intermediate stages we needed more integrals than those calculated for [19, 20]. They were calculated with the methods of [29] and are given in App. D.

$$
\begin{aligned}
& B(s, t, u)=x_{2}^{3}\left[B_{P}(s, t, u)+B_{S}(s, t-u)+B_{S}(u, t-s)+B_{T}(t)\right] \\
& C(s, t, u)=x_{2}^{3}\left[C_{P}(s, t, u)+C_{S}(s)+C_{T}(t)+C_{T}(u)\right] .
\end{aligned}
$$




\begin{tabular}{|c|c|c|}
\hline \multicolumn{3}{|r|}{ QCD: $S U(n) \times S U(n) / S U(n)$} \\
\hline$B_{P}(s, t, u)$ & $\begin{array}{l}\alpha_{1} \\
\alpha_{2} \\
\alpha_{3} \\
\alpha_{4}\end{array}$ & $\begin{array}{c}\frac{2}{n} L+\frac{2}{n} \pi_{16}+16 L_{8}^{r}+16 L_{0}^{r}-\frac{2}{3} n L-\frac{5}{9} n \pi_{16} \\
-4 L_{5}^{r}-16 L_{0}^{r}+\frac{5}{12} n L+\frac{11}{36} n \pi_{16} \\
L_{3}^{r}+4 L_{0}^{r}-\frac{1}{16} n L-\frac{1}{24} n \pi_{16} \\
L_{3}^{r}-\frac{1}{48} n L-\frac{1}{36} n \pi_{16}\end{array}$ \\
\hline$C_{P}(s, t, u)$ & $\begin{array}{l}\beta_{1} \\
\beta_{2} \\
\beta_{3} \\
\beta_{4}\end{array}$ & $\begin{array}{c}32\left(L_{1}^{r}-L_{4}^{r}+L_{6}^{r}\right)-\frac{2}{n^{2}}\left(L+\pi_{16}\right) \\
16 L_{4}^{r}-32 L_{1}^{r} \\
-\frac{3}{8} L+2 L_{2}^{r}+8 L_{1}^{r}-\frac{3}{8} \pi_{16} \\
2 L_{2}^{r}-\frac{1}{8} L-\frac{1}{8} \pi_{16}\end{array}$ \\
\hline \multicolumn{3}{|r|}{ Adjoint: $S U(2 n) / S O(2 n)$} \\
\hline$B_{P}(s, t, u)$ & $\begin{array}{l}\alpha_{1} \\
\alpha_{2} \\
\alpha_{3} \\
\alpha_{4}\end{array}$ & $\begin{array}{c}16\left(L_{0}^{r}+L_{8}^{r}\right)-\left(\frac{7}{6}+\frac{2 n}{3}-\frac{1}{n}\right) L-\left(\frac{19}{18}+\frac{5 n}{9}-\frac{1}{n}\right) \pi_{16} \\
-16 L_{0}^{r}-4 L_{5}^{r}+\left(\frac{5 n}{12}+\frac{2}{3}\right) L+\left(\frac{5}{9}+\frac{11 n}{36}\right) \pi_{16} \\
4 L_{0}^{r}+L_{3}^{r}-\left(\frac{1}{8}+\frac{n}{16}\right) L-\left(\frac{5}{48}+\frac{n}{24}\right) \pi_{16} \\
L_{3}^{r}+\left(\frac{1}{24}-\frac{n}{48}\right) L+\left(\frac{5}{144}-\frac{n}{36}\right) \pi_{16} \\
\end{array}$ \\
\hline$C_{P}(s, t, u)$ & $\begin{array}{l}\beta_{1} \\
\beta_{2} \\
\beta_{3} \\
\beta_{4}\end{array}$ & $\begin{array}{c}32\left(L_{1}^{r}-L_{4}^{r}+L_{6}^{r}\right)-\frac{1}{2 n^{2}}\left(L+\pi_{16}\right) \\
16\left(L_{4}^{r}-2 L_{1}^{r}\right) \\
8 L_{1}^{r}+2 L_{2}^{r}-\frac{3}{16} \pi_{16}-\frac{3}{16} L \\
2 L_{2}^{r}-\frac{1}{16} L-\frac{1}{16} \pi_{16} \\
\end{array}$ \\
\hline \multicolumn{3}{|r|}{ Two-colour: $S U(2 N) / S p(2 N)$} \\
\hline$B_{P}(s, t, u)$ & $\begin{array}{l}\alpha_{1} \\
\alpha_{2} \\
\alpha_{3} \\
\alpha_{4}\end{array}$ & $\begin{array}{c}16\left(L_{0}^{r}+L_{8}^{r}\right)+\left(\frac{7}{6}+\frac{1}{n}-\frac{2 n}{3}\right) L+\left(\frac{19}{18}-\frac{5 n}{9}+\frac{1}{n}\right) \pi_{16} \\
-16 L_{0}^{r}-4 L_{5}^{r}+\left(\frac{5 n}{12}-\frac{2}{3}\right) L+\left(\frac{5}{9}-\frac{11 n}{36}\right) \pi_{16} \\
4 L_{0}^{r}+L_{3}^{r}+\left(\frac{1}{8}-\frac{n}{16}\right) L+\left(\frac{5}{48}-\frac{n}{24}\right) \pi_{16} \\
L_{3}^{r}-\left(\frac{1}{24}+\frac{n}{48}\right) L-\left(\frac{5}{144}+\frac{n}{36}\right) \pi_{16} \\
\end{array}$ \\
\hline$C_{P}(s, t, u)$ & $\begin{array}{l}\beta_{1} \\
\beta_{2} \\
\beta_{3} \\
\beta_{4}\end{array}$ & $\begin{array}{c}32\left(L_{1}^{r}-L_{4}^{r}+L_{6}^{r}\right)-\frac{1}{2 n^{2}}\left(L+\pi_{16}\right) \\
16\left(L_{4}^{r}-2 L_{1}^{r}\right) \\
8 L_{1}^{r}+2 L_{2}^{r}-\frac{3}{16} \pi_{16}-\frac{3}{16} L \\
2 L_{2}^{r}-\frac{1}{16} L-\frac{1}{16} \pi_{16}\end{array}$ \\
\hline
\end{tabular}

Table 5: The next-to-leading results for all three cases for the polynomial part. The coefficients are defined in (74). 


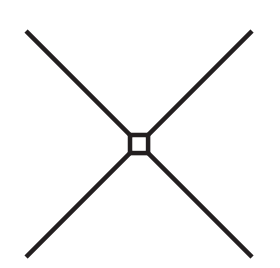

(5)

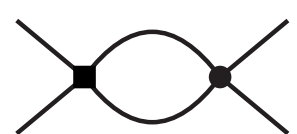

(6)

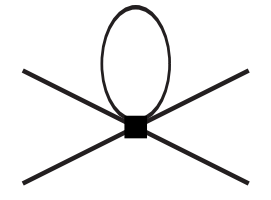

(7)

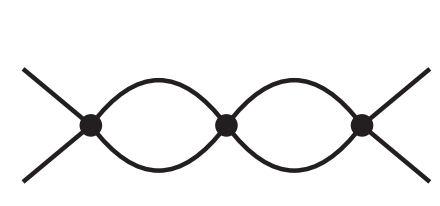

(8)

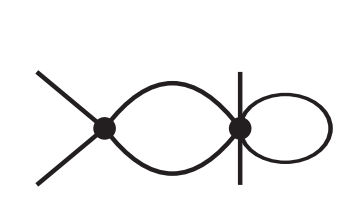

(9)

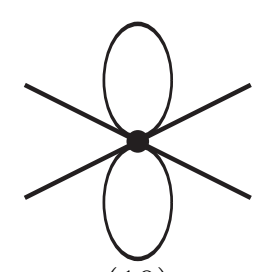

(10)

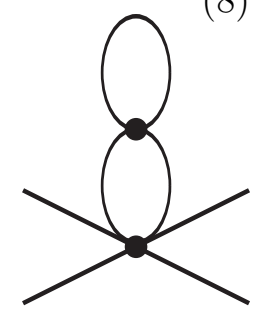

(11)

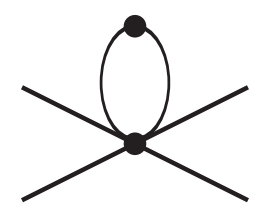

(12)

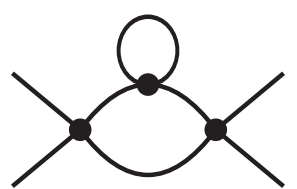

(13)

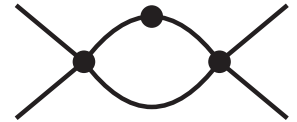

(14)

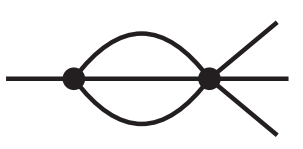

(15)

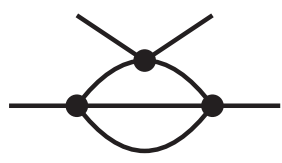

(16)

Figure 2: The next-to-next-to leading order diagrams for $\pi \pi \rightarrow \pi \pi$. The filled circle a vertex from $\mathcal{L}_{2}$, The filled square is a vertex from $\mathcal{L}_{4}$, and the open square is a vertex from $\mathcal{L}_{6}$. 


\begin{tabular}{|c|c|}
\hline \multicolumn{2}{|c|}{ QCD: $S U(n) \times S U(n) / S U(n)$} \\
\hline$B_{S}(s, t-u)$ & $\bar{J}(s)\left[-\frac{1}{n}+\frac{n}{16} s^{2}+\frac{n}{12}\left(1-\frac{s}{4}\right)(t-u)\right]$ \\
$B_{T}(t)$ & 0 \\
\hline$C_{S}(s)$ & $\bar{J}(s)\left(\frac{2}{n^{2}}+\frac{1}{4} s^{2}\right)$ \\
$C_{T}(t)$ & $\frac{1}{4} \bar{J}(t)(t-2)^{2}$ \\
\hline \multicolumn{2}{|c|}{ Adjoint: $S U(2 n) / S O(2 n)$} \\
\hline$B_{S}(s, t-u)$ & $\bar{J}(s)\left[-\frac{1}{2 n}+\frac{s}{4}+\frac{1}{16}(n-1) s^{2}+\frac{1}{12}(n+1)\left(1-\frac{s}{4}\right)(t-u)\right]$ \\
$B_{T}(t)$ & $\frac{1}{8} \bar{J}(t)(t-2)^{2}$ \\
\hline$C_{S}(s)$ & $\bar{J}(s)\left(\frac{1}{2 n^{2}}+\frac{1}{8} s^{2}\right)$ \\
$C_{T}(t)$ & $\frac{1}{8} \bar{J}(t)(t-2)^{2}$ \\
\hline \multicolumn{2}{|c|}{$\left.\left.(t)=\frac{1}{4}\right)(t-u)\right]$} \\
\hline$B_{S}(s, t-u)$ & $\bar{J}(s)\left[-\frac{1}{2 n}-\frac{1}{4} s+\frac{1}{16}(n+1) s^{2}+\frac{1}{12}(n-1)\left(1-\frac{s}{8}\right)(t)(t-2)^{2}\right.$ \\
$B_{T}(t)$ & $-\frac{1}{8} \bar{J}(s)\left(\frac{1}{2 n^{2}}+\frac{1}{8} s^{2}\right)$ \\
\hline$C_{S}(s)$ & $\frac{1}{8} \bar{J}(t)(t-2)^{2}$ \\
$C_{T}(t)$ &
\end{tabular}

Table 6: The next-to-leading results for all three cases for the unitarity correction.

The polynomial parts we rewrite using (28) in their simplest form satisfying the symmetry constraints (31):

$$
\begin{aligned}
& B_{P}(s, t, u)=\gamma_{1}+\gamma_{2} t+\gamma_{3} t^{2}+\gamma_{4}(s-u)^{2}+\gamma_{5} t^{3}+\gamma_{6} t(s-u)^{2}, \\
& C_{P}(s, t, u)=\delta_{1}+\delta_{2} s+\delta_{3} s^{2}+\delta_{4}(t-u)^{2}+\delta_{5} s^{3}+\delta_{6} s(t-u)^{2} .
\end{aligned}
$$

The coefficients in these polynomials as well as the functions in (75) are given in App. A. The FORM expressions can be downloaded from [30]. We stress once more that the result is fully analytical and expressed in terms of $L$ and $\bar{J}$.

\section{$5 \quad$ Scattering lengths}

The threshold parameters of general meson meson scattering are defined similar to those of $\pi \pi$ scattering. First we calculate the amplitudes for the different channels using (57), (64) and (69) for each of the possible intermediate states of representation or channels $I$.

The scattering amplitude for each channel $I$ can be projected out using the partial wave expansion

$$
T_{\ell}^{I}(s)=\frac{1}{64 \pi} \int_{-1}^{1} d(\cos \theta) P_{\ell}(\cos \theta) T_{I}(s, t, u) .
$$

Near the threshold $s=4$, we can expand the amplitude above the threshold using $s=4\left(1+q^{2} / M_{\pi}^{2}\right)$ in the small three-momentum $q$.

$$
\operatorname{Re} T_{\ell}^{I}(s)=q^{2 \ell}\left[a_{\ell}^{I}+q^{2} b_{\ell}^{I}+O\left(q^{4}\right)\right]
$$


where $a_{\ell}^{I}$ is the scattering length, and $b_{\ell}^{I}$ is the slope.

In App. C, we give the expressions of the lowest partial wave scattering length for each channel in all three cases. As mentioned in Sect. 3, some channels are symmetric under $A \leftrightarrow B$, hence the lowest order partial wave is $\ell=0$. The other channels are antisymmetric under $A \leftrightarrow B$, so that the lowest order partial wave is $\ell=1$. $A$ and $B$ are the incoming mesons here using the notation of Sect. 3 ,

For the purpose of illustration, we plot the scattering length for the singlet and the fully-symmetric (fully-antisymmetric) channels as a function of the physical meson mass $M_{\text {phys }}^{2}$. Since currently we do not have knowledge for the values of the low energy constants for these, we take the values of the $L_{i}^{r}$ of fit 10 of [31 for the complex or QCD case and half that for the other two as suggested by the large $n$ relations discussed below. The values of the NNLO LECs we simply put to zero. We also choose the subtraction scale $\mu=0.77 \mathrm{GeV}$ and the physical decay constant $F_{\text {phys }}=0.0924 \mathrm{GeV}$.

The singlet case for the complex case is shown in Fig. 3. We have divided the scattering length by $n$ to make the lowest order similar for all cases. Plotted are $n=2, \ldots, 5$. One can see that for a given $M_{\text {phys }}^{2}$ the convergence gets progressively worse for larger $n$. For $n=2$ this corresponds to $a_{0}^{0}$.

The fully symmetric case case for the complex case is shown in Fig. 4. Plotted are $n=2, \ldots, 5$. One can see that for a given $M_{\text {phys }}^{2}$ the convergence gets progressively worse for larger $n$. For $n=2$ this corresponds to $a_{0}^{2}$. The lowest order is independent of $n$. The NLO order is only mildly dependent on $n$ while the NNLO part grows fast with $n$.

\subsection{Large $n$ behaviour}

Looking at the lowest order expressions in App. C we notice immediately that the large $n$ behaviour for the scattering lengths fall in three classes.

The scattering length is of order $n$ for the singlet and symmetric and asymmetric representation and what is more they are clearly related for the three cases:

$$
\begin{aligned}
& \left.a_{0}^{I}\right|_{\text {complex }}=\left.a_{0}^{I}\right|_{\text {real }}=\left.a_{0}^{I}\right|_{\text {pseudoreal }}={ }_{L O} \frac{x_{2}}{\pi} \frac{n}{8}, \\
& \left.a_{0}^{S}\right|_{\text {complex }}=\left.a_{0}^{S}\right|_{\text {real }}=\left.a_{0}^{A}\right|_{\text {pseudoreal }}={ }_{L O} \frac{x_{2}}{\pi} \frac{n}{16}, \\
& \left.a_{1}^{A}\right|_{\text {complex }}=\left.a_{1}^{A}\right|_{\text {real }}=\left.a_{1}^{S}\right|_{\text {pseudoreal }}={ }_{L O} \frac{x_{2}}{\pi} \frac{n}{48},
\end{aligned}
$$

The symbol $=_{L O}$ means equality at lowest order. Do the relations (79, 81 remain valid at higher orders? If we choose $F_{\text {phys }}^{2} \propto n$ and $M_{\text {phys }}^{2}$ independent of $n$, we find that it is indeed the case for (80,81). For (79) it is true, provided we set the NLO LECs $L_{i}^{r}$ of the real and pseudoreal case to half those of the complex case and the NNLO coefficients $K_{i}$ to $1 / 4$ the complex case. The contributions are nonzero at the three orders for all of them. The subleading orders in $1 / n$ are different.

A second class is those which are order 1 in the coefficient of $x_{2} / \pi$ at lowest order. For these we find

$$
\left.a_{0}^{S S}\right|_{\text {complex }}=\left.a_{0}^{F S}\right|_{\text {real }}=\left.2 a_{0}^{M S}\right|_{\text {pseudoreal }}={ }_{L O} \frac{x_{2}}{\pi} \frac{-1}{16}
$$



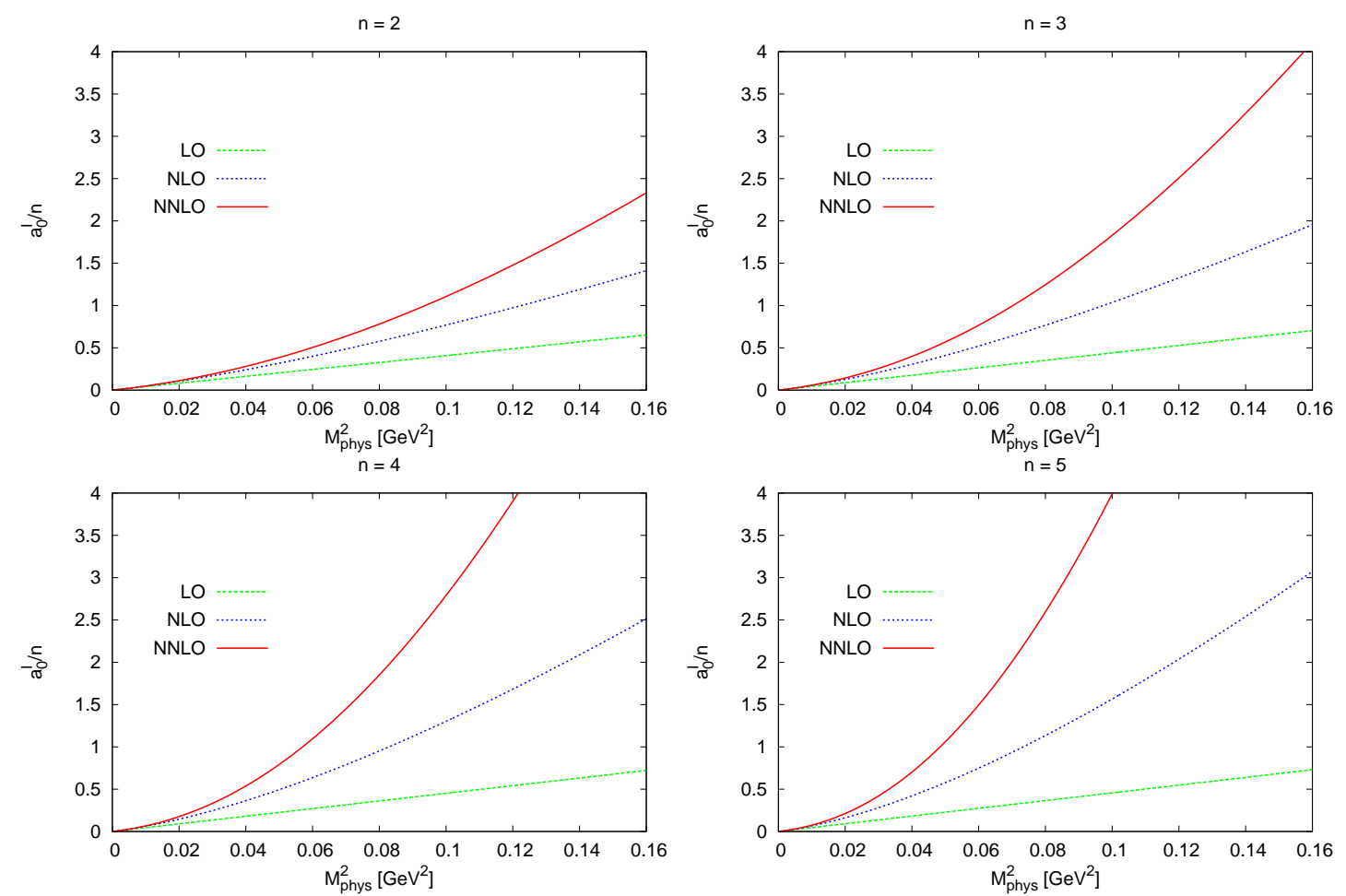

Figure 3: Scattering length $a_{0}^{I} / n$ for the complex or QCD case, $S U(n) \times$ $S U(n) / S U(n)$.
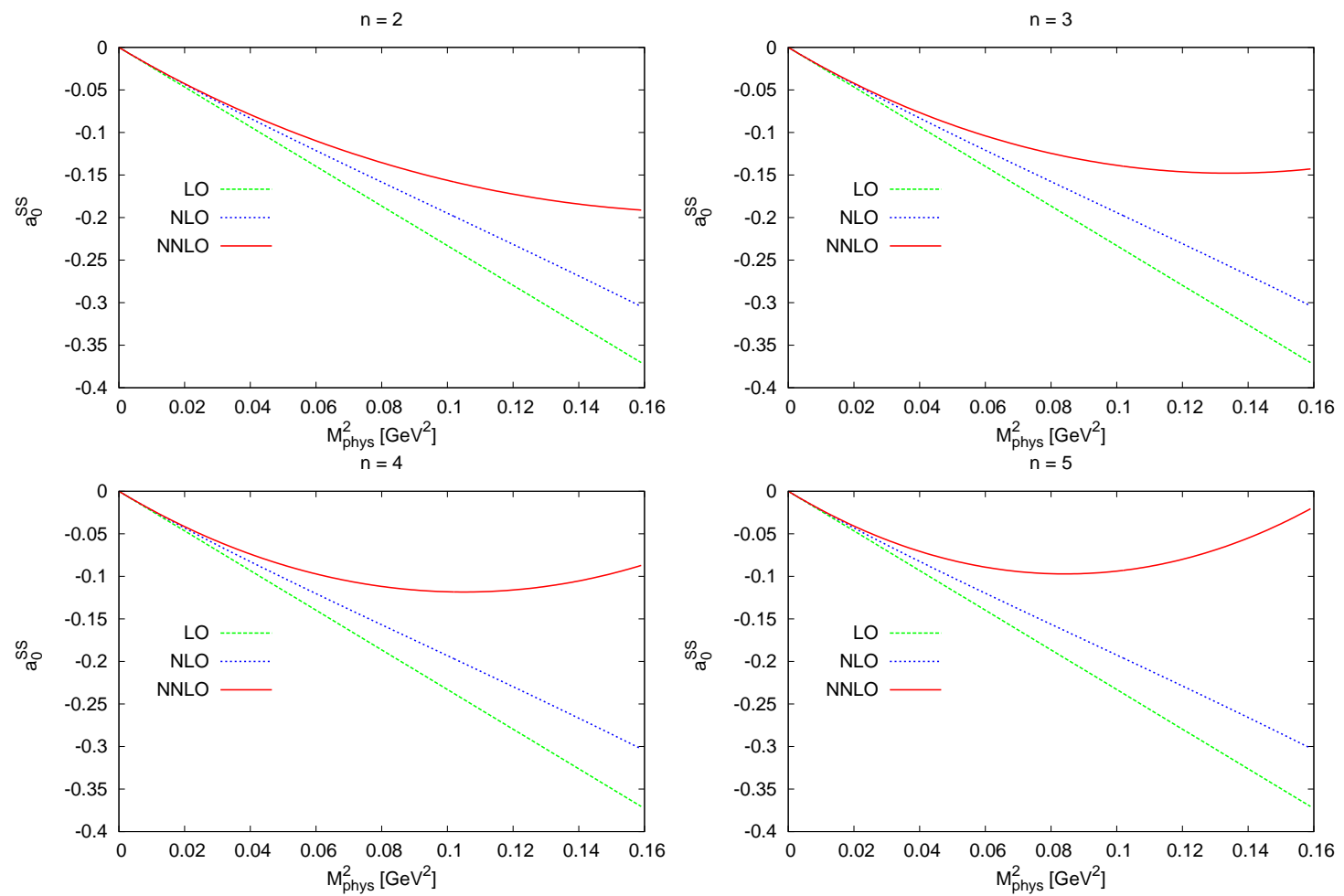

Figure 4: Scattering length $a_{0}^{S S}$ for the complex or QCD case, $S U(n) \times S U(n) / S U(n)$. 

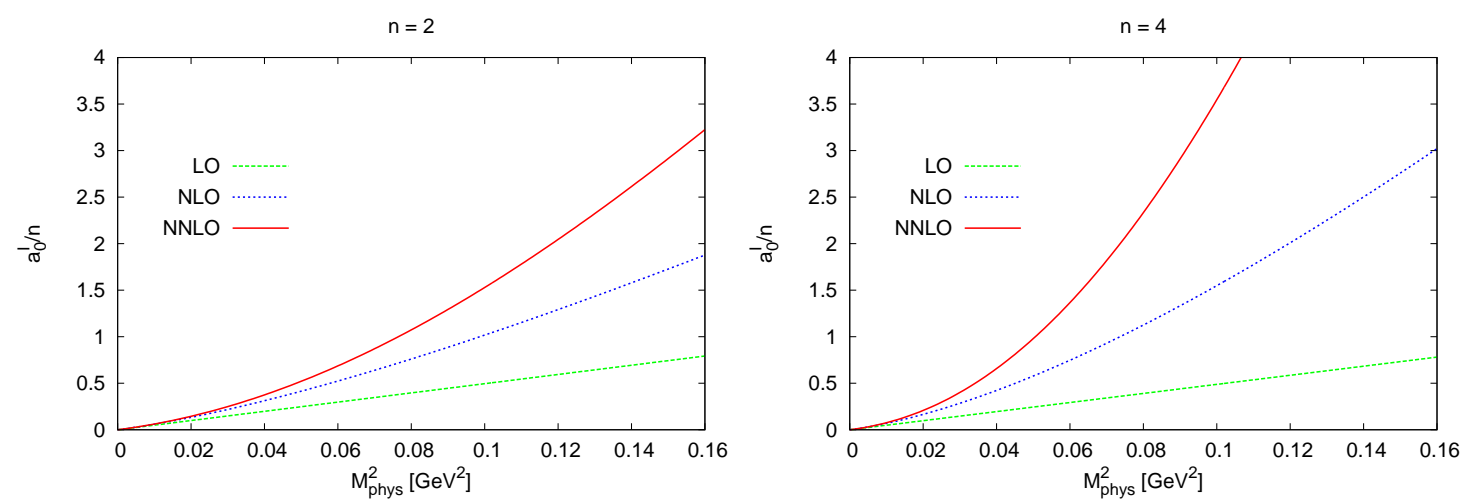

Figure 5: Scattering length of $a_{0}^{I} / n$ for the real or adjoint case, $S U(2 n) / S O(2 n)$.
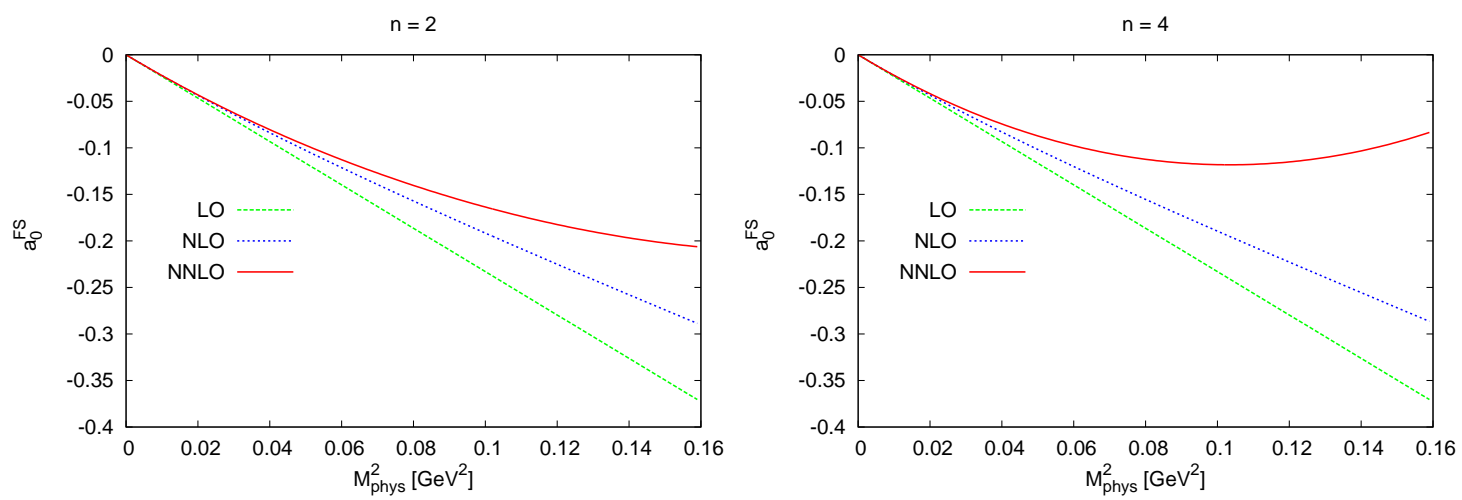

Figure 6: Scattering length of $a_{0}^{F S}$ for the real or adjoint case, $S U(2 n) / S O(2 n)$.
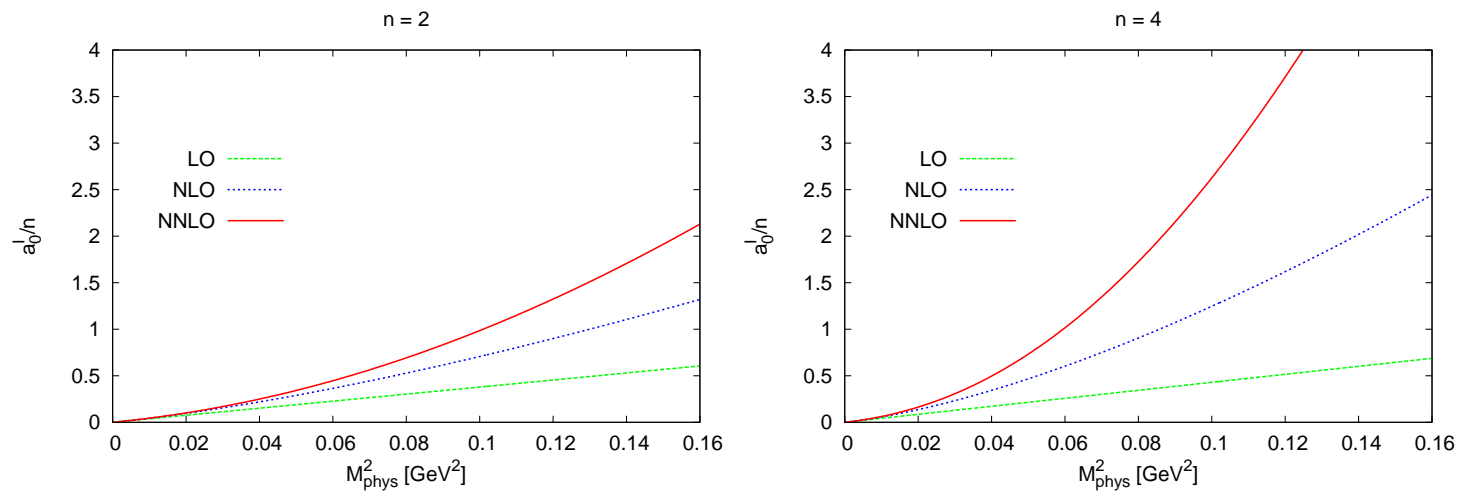

Figure 7: Scattering length of $a_{0}^{I} / n$ for the pseudoreal or two-colour case, $S U(2 n) / S p(2 n)$. 

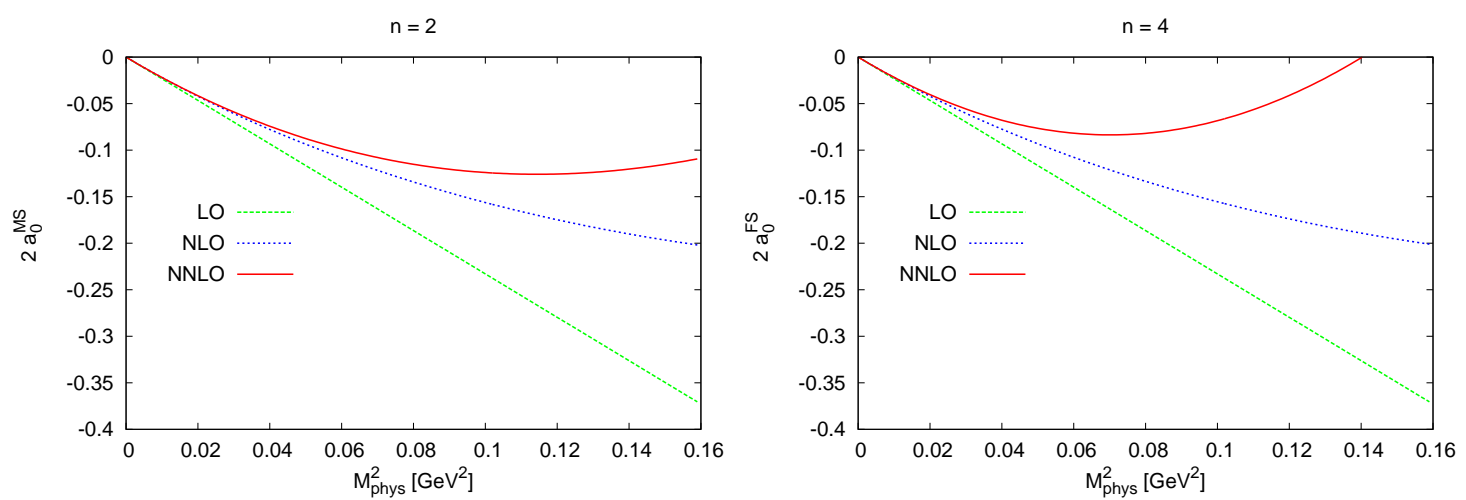

Figure 8: Scattering length $2 a_{0}^{M S}$ for the pseudoreal or two-colour case, $S U(2 n) / S p(2 n)$. The factor of 2 included is because of the large $n$ relation (82).

$$
\left.a_{0}^{A A}\right|_{\text {complex }}=\left.2 a_{0}^{M S}\right|_{\text {real }}=\left.a_{0}^{F A}\right|_{\text {pseudoreal }}={ }_{L O} \frac{x_{2}}{\pi} \frac{1}{16}
$$

We do indeed find that the relations are also satisfied at NLO and NNLO. In fact none of the scattering lengths in (82) has a leading $n$ NLO correction to the lowest order result. This can be clearly seen in Fig. 4 where the NLO result is very similar in all plots.

The third class is the amplitudes that vanish at lowest order

$$
\left.a_{1}^{S A}\right|_{\text {complex }}=\left.a_{1}^{A S}\right|_{\text {complex }}=\left.2 a_{1}^{M A}\right|_{\text {real }}=\left.2 a_{1}^{M A}\right|_{\text {pseudoreal }}={ }_{L O} 0 .
$$

These are always suppressed by two powers of $n$ compared to the first set of scattering lengths also at NLO and NNLO. The relations (84) are satisfied at NLO with same identifications of the LECs as above and almost at NNLO. The only terms that do not satisfy the relation are proportional to $L_{4}^{r} L_{6}^{r}$.

By comparing the plots shown one sees that the large $n$ relations do predict the general behaviour but for $n=2$ and $n=4$ are not that accurate.

\section{Conclusions}

In this work we have presented the calculation of general meson-meson scattering for $n$ flavours in a complex, real or pseudoreal representation of a strongly interaction gauge group. These are also referred to as QCD, Adjoint QCD and Two-color QCD and have as symmetry breaking patterns $S U(n) \times S U(n) / S U(n), S U(2 n) / S O(2 n)$ and $S U(2 n) / S p(2 n)$

We first reviewed the effective field theories of these three different cases. Those theories can be written in a very similar form as discussed earlier [1]. We have extended the methods used for $\pi \pi$ scattering in ChPT [20] to all the present cases. At intermediate stages some more integrals showed up, we have calculated them and they are tabulated in an appendix.

The amplitude can in general be written in terms of two invariant amplitudes which we called $B(s, t, u)$ and $C(s, t, u)$. These amplitudes can be written in terms of simpler functions and we have given their fully analytical expressions to NNLO. 
Since the long term use of our work is the study of scattering on the lattice for these alternative theories we have discussed the group theory involved and all the possible intermediate channels. We have derived the amplitudes in all these channel as a function of the invariant $B$ and $C$ functions.

The expressions for the different channels we have not shown explicitly but we included expressions for scattering length of the lowest partial wave in all channels. We presented a few representative numerical results for the scattering lengths and discussed a series of relations between the different theories in the limit of a large number of flavours $n$.

\section{Acknowledgements}

This work is supported in part by the European Community-Research Infrastructure Integrating Activity "Study of Strongly Interacting Matter" (HadronPhysics2, Grant Agreement n. 227431) and the Swedish Research Council grants 621-2008-4074 and 621-2011-3326. This work heavily used FORM [32].

\section{A Next-to-next-to leading order result}

\section{A.1 Complex or QCD}

$$
\begin{aligned}
& B_{S}(s, t-u)=k_{4}(s)\left\{\frac{n_{f}^{2}}{12}+\frac{2}{n_{f}^{2}}\right\}(t-u) \\
& +k_{3}(s)\left\{\frac{s^{2} n_{f}^{2}}{48}-\frac{s n_{f}^{2}}{18}-\frac{1}{48} s(t-u) n_{f}^{2}+\frac{(t-u) n_{f}^{2}}{36}-\frac{(t-u)}{6}+\frac{1}{3}-\frac{s}{3 n_{f}^{2}}\right. \\
& \left.+\frac{(t-u)}{3 n_{f}^{2}}-\frac{8}{3 n_{f}^{2}}\right\} \\
& +k_{2}(s)\left\{\frac{n_{f}^{2} s^{3}}{64}+\frac{s^{3}}{16}-\frac{1}{576} n_{f}^{2}(t-u) s^{2}-\frac{3 s^{2}}{8}+\frac{1}{72} n_{f}^{2}(t-u) s-\frac{n_{f}^{2}(t-u)}{36}+\frac{2}{n_{f}^{2}}\right\} \\
& +k_{1}(s)\left\{\frac{n_{f}^{2} s^{3}}{576}+\frac{s^{3}}{24}-\frac{17 n_{f}^{2} s^{2}}{288}+\frac{1}{576} n_{f}^{2}(t-u) s^{2}-\frac{s^{2}}{4}+\frac{n_{f}^{2} s}{12}-\frac{1}{96} n_{f}^{2}(t-u) s\right. \\
& \left.-\frac{(t-u) s}{24}+\frac{s}{2 n_{f}^{2}}+\frac{7 s}{12}-\frac{n_{f}^{2}(t-u)}{48}+\frac{(t-u)}{4}+\frac{4}{n_{f}^{2}}-\frac{1}{2}\right\} \\
& +\bar{J}(s)\left\{-\frac{5}{144} L n_{f}^{2} s^{3}-\frac{5 L s^{3}}{24}+\frac{4 L_{1}^{r} s^{3}}{3}+\frac{8 L_{2}^{r} s^{3}}{3}+\frac{2}{3} L_{0}^{r} n_{f} s^{3}+\frac{4}{3} L_{3}^{r} n_{f} s^{3}\right. \\
& +\frac{17}{576} n_{f}^{2} \pi_{16} s^{3}+\frac{17 \pi_{16} s^{3}}{72}+\frac{7}{144} L n_{f}^{2} s^{2}+\frac{4 L s^{2}}{3}-\frac{8 L_{1}^{r} s^{2}}{3}-\frac{28 L_{2}^{r} s^{2}}{3}-4 L_{4}^{r} s^{2} \\
& -\frac{4}{3} L_{0}^{r} n_{f} s^{2}-\frac{14}{3} L_{3}^{r} n_{f} s^{2}+L_{5}^{r} n_{f} s^{2}-\frac{35}{144} n_{f}^{2} \pi_{16} s^{2}-\frac{13 \pi_{16} s^{2}}{9}+\frac{2}{3} L_{1}^{r}(t-u) s^{2} \\
& -\frac{1}{3} L_{2}^{r}(t-u) s^{2}-\frac{1}{3} L_{0}^{r} n_{f}(t-u) s^{2}+\frac{1}{6} L_{3}^{r} n_{f}(t-u) s^{2}-\frac{1}{432} n_{f}^{2} \pi_{16}(t-u) s^{2}
\end{aligned}
$$




$$
\begin{aligned}
& -\frac{40 L_{0}^{r} s^{2}}{3 n_{f}}-\frac{40 L_{3}^{r} s^{2}}{3 n_{f}}-\frac{5}{36} L n_{f}^{2} s-\frac{5 L s}{3}+\frac{16 L_{1}^{r} s}{3}+\frac{32 L_{2}^{r} s}{3}+16 L_{6}^{r} s+\frac{8 L_{0}^{r} n_{f} s}{3} \\
& +\frac{16 L_{3}^{r} n_{f} s}{3}-4 L_{5}^{r} n_{f} s+8 L_{8}^{r} n_{f} s+\frac{5}{12} n_{f}^{2} \pi_{16} s+\frac{2 \pi_{16} s}{n_{f}^{2}}+\frac{65 \pi_{16} s}{36} \\
& +\frac{1}{48} L n_{f}^{2}(t-u) s+\frac{L(t-u) s}{12}-\frac{8 L_{1}^{r}(t-u) s}{3}+\frac{4 L_{2}^{r}(t-u) s}{3}-\frac{4 L_{4}^{r}(t-u) s}{3} \\
& +\frac{4}{3} L_{0}^{r} n_{f}(t-u) s-\frac{2}{3} L_{3}^{r} n_{f}(t-u) s-\frac{1}{3} L_{5}^{r} n_{f}(t-u) s+\frac{17}{216} n_{f}^{2} \pi_{16}(t-u) s \\
& -\frac{5 \pi_{16}(t-u) s}{72}+\frac{128 L_{0}^{r} s}{3 n_{f}}+\frac{128 L_{3}^{r} s}{3 n_{f}}-\frac{L s}{n_{f}^{2}}+\frac{8 L}{3}+\frac{20 \pi_{16}}{n_{f}^{2}}-\frac{20 \pi_{16}}{9} \\
& -\frac{1}{12} \operatorname{Ln}_{f}^{2}(t-u)-\frac{L(t-u)}{3}+\frac{16 L_{4}^{r}(t-u)}{3}+\frac{4 L_{5}^{r} n_{f}(t-u)}{3} \\
& -\frac{11}{48} n_{f}^{2} \pi_{16}(t-u)-\frac{\pi_{16}(t-u)}{2 n_{f}^{2}}+\frac{10 \pi_{16}(t-u)}{9}-\frac{160 L_{0}^{r}}{3 n_{f}}-\frac{160 L_{3}^{r}}{3 n_{f}}+\frac{32 L_{5}^{r}}{n_{f}} \\
& \left.-\frac{96 L_{8}^{r}}{n_{f}}-\frac{12 L}{n_{f}^{2}}\right\} \\
& B_{T}(t)=k_{3}(t)\left\{\frac{2 t}{3 n_{f}^{2}}+\frac{t}{3}-\frac{4}{3 n_{f}^{2}}-\frac{2}{3}\right\}+k_{2}(t)\left\{-\frac{t^{3}}{8}+\frac{3 t^{2}}{4}-\frac{3 t}{2}+1\right\} \\
& +k_{1}(t)\left\{-\frac{t^{3}}{12}+\frac{t^{2}}{2}-\frac{t}{n_{f}^{2}}-\frac{7 t}{6}+\frac{2}{n_{f}^{2}}+1\right\} \\
& +\bar{J}(t)\left\{\frac{5 L t^{3}}{12}-\frac{8 L_{1}^{r} t^{3}}{3}-\frac{16 L_{2}^{r} t^{3}}{3}-\frac{17 \pi_{16} t^{3}}{36}-\frac{8 L t^{2}}{3}+\frac{32 L_{1}^{r} t^{2}}{3}+\frac{88 L_{2}^{r} t^{2}}{3}+8 L_{4}^{r} t^{2}\right. \\
& +\frac{26 \pi_{16} t^{2}}{9}+\frac{19 L t}{3}-\frac{64 L_{1}^{r} t}{3}-\frac{176 L_{2}^{r} t}{3}-16 L_{4}^{r} t-32 L_{6}^{r} t-\frac{4 \pi_{16} t}{n_{f}^{2}}-\frac{64 \pi_{16} t}{9} \\
& \left.+\frac{2 L t}{n_{f}^{2}}-\frac{16 L}{3}+\frac{64 L_{1}^{r}}{3}+\frac{128 L_{2}^{r}}{3}+64 L_{6}^{r}+\frac{8 \pi_{16}}{n_{f}^{2}}+\frac{58 \pi_{16}}{9}-\frac{4 L}{n_{f}^{2}}\right\} \\
& C_{S}(s)=k_{3}(s)\left\{\frac{n_{f} s^{2}}{12}-\frac{5 n_{f} s}{9}+\frac{2 s}{3 n_{f}}+\frac{16}{3 n_{f}^{3}}\right\} \\
& +k_{2}(s)\left\{\frac{3 n_{f} s^{3}}{16}-\frac{6}{n_{f}^{3}}\right\}+k_{1}(s)\left\{\frac{13 n_{f} s^{3}}{144}-\frac{23 n_{f} s^{2}}{72}+\frac{5 n_{f} s}{6}-\frac{2 s}{n_{f}}-\frac{8}{n_{f}^{3}}\right\} \\
& +\bar{J}(s)\left\{\frac{8 L_{0}^{r} s^{3}}{3}+\frac{16 L_{3}^{r} s^{3}}{3}-\frac{5}{9} L n_{f} s^{3}+8 L_{1}^{r} n_{f} s^{3}+\frac{8}{3} L_{2}^{r} n_{f} s^{3}+\frac{85}{144} n_{f} \pi_{16} s^{3}\right. \\
& -\frac{16 L_{0}^{r} s^{2}}{3}-\frac{56 L_{3}^{r} s^{2}}{3}+4 L_{5}^{r} s^{2}+\frac{19}{36} L n_{f} s^{2}-32 L_{1}^{r} n_{f} s^{2}-\frac{16}{3} L_{2}^{r} n_{f} s^{2} \\
& +16 L_{4}^{r} n_{f} s^{2}-\frac{7}{6} n_{f} \pi_{16} s^{2}-\frac{16 L_{1}^{r} s^{2}}{n_{f}}-\frac{16 L_{2}^{r} s^{2}}{3 n_{f}}+\frac{80 L_{0}^{r} s^{2}}{3 n_{f}^{2}}+\frac{80 L_{3}^{r} s^{2}}{3 n_{f}^{2}}+\frac{32 L_{0}^{r} s}{3} \\
& +\frac{64 L_{3}^{r} s}{3}-16 L_{5}^{r} s+32 L_{8}^{r} s-\frac{11 L n_{f} s}{9}+32 L_{1}^{r} n_{f} s+\frac{32 L_{2}^{r} n_{f} s}{3}-32 L_{4}^{r} n_{f} s \\
& +32 L_{6}^{r} n_{f} s+\frac{35 n_{f} \pi_{16} s}{9}-\frac{7 \pi_{16} s}{n_{f}}+\frac{4 L s}{n_{f}}+\frac{64 L_{1}^{r} s}{n_{f}}+\frac{32 L_{2}^{r} s}{3 n_{f}}-\frac{32 L_{4}^{r} s}{n_{f}}
\end{aligned}
$$




$$
\begin{aligned}
& -\frac{256 L_{0}^{r} s}{3 n_{f}^{2}}-\frac{256 L_{3}^{r} s}{3 n_{f}^{2}}-\frac{44 \pi_{16}}{n_{f}^{3}}-\frac{4 L}{n_{f}}-\frac{64 L_{1}^{r}}{n_{f}}-\frac{64 L_{2}^{r}}{3 n_{f}}+\frac{64 L_{4}^{r}}{n_{f}}-\frac{64 L_{6}^{r}}{n_{f}} \\
& \left.+\frac{320 L_{0}^{r}}{3 n_{f}^{2}}+\frac{320 L_{3}^{r}}{3 n_{f}^{2}}-\frac{64 L_{5}^{r}}{n_{f}^{2}}+\frac{192 L_{8}^{r}}{n_{f}^{2}}+\frac{28 L}{n_{f}^{3}}\right\} \\
C_{T}(t)= & k_{3}(t)\left\{\frac{n_{f} t^{2}}{12}-\frac{2 n_{f} t}{9}-\frac{2 t}{3 n_{f}}+\frac{n_{f}}{9}+\frac{4}{3 n_{f}}\right\} \\
+k_{1}(t) & \left\{-\frac{5 n_{f} t^{3}}{144}+\frac{n_{f} t^{2}}{8}-\frac{n_{f} t}{36}+\frac{t}{n_{f}}-\frac{n_{f}}{6}-\frac{2}{n_{f}}\right\} \\
+\bar{J}(t) & \left\{-4 L_{0}^{r} t^{3}-\frac{4 L_{3}^{r} t^{3}}{3}+\frac{5}{72} L n_{f} t^{3}-\frac{17}{144} n_{f} \pi_{16} t^{3}+24 L_{0}^{r} t^{2}+\frac{16 L_{3}^{r} t^{2}}{3}+4 L_{5}^{r} t^{2}\right. \\
& -\frac{11}{18} L n_{f} t^{2}+\frac{1}{9} n_{f} \pi_{16} t^{2}-48 L_{0}^{r} t-\frac{32 L_{3}^{r} t}{3}-8 L_{5}^{r} t-16 L_{8}^{r} t+\frac{31 L n_{f} t}{18} \\
+ & \frac{29 n_{f} \pi_{16} t}{36}+\frac{4 \pi_{16} t}{n_{f}}-\frac{2 L t}{n_{f}}+32 L_{0}^{r}+\frac{32 L_{3}^{r}}{3}+32 L_{8}^{r}-\frac{14 L n_{f}}{9}-\frac{10 n_{f} \pi_{16}}{9} \\
& \left.-\frac{8 \pi_{16}}{n_{f}}+\frac{4 L}{n_{f}}\right\}
\end{aligned}
$$

\section{A.2 Real or adjoint}

$$
\begin{aligned}
B_{S}(s, t-u) & =k_{4}(s)\left\{\frac{(t-u) n^{2}}{12}-\frac{(t-u) n}{12}-\frac{(t-u)}{6}-\frac{(t-u)}{2 n}+\frac{(t-u)}{2 n^{2}}\right\} \\
+k_{3}(s) & \left\{\frac{s^{2} n^{2}}{48}-\frac{s n^{2}}{18}-\frac{1}{48} s(t-u) n^{2}+\frac{(t-u) n^{2}}{36}-\frac{s n}{24}-\frac{s(t-u) n}{24}+\frac{(t-u) n}{72}\right. \\
& -\frac{n}{9}-\frac{s^{2}}{48}+\frac{s}{18}-\frac{s(t-u)}{48}-\frac{7(t-u)}{72}-\frac{1}{36}+\frac{s}{12 n}-\frac{(t-u)}{12 n}+\frac{1}{2 n}-\frac{s}{12 n^{2}} \\
+ & \left.\frac{(t-u)}{12 n^{2}}-\frac{2}{3 n^{2}}\right\} \\
+k_{2}(s) & \left\{\frac{n^{2} s^{3}}{64}-\frac{n s^{3}}{64}+\frac{3 s^{3}}{64}+\frac{3 n s^{2}}{32}-\frac{1}{576} n^{2}(t-u) s^{2}-\frac{1}{288} n(t-u) s^{2}\right. \\
& -\frac{(t-u) s^{2}}{576}-\frac{9 s^{2}}{32}+\frac{1}{72} n^{2}(t-u) s+\frac{n(t-u) s}{36}+\frac{(t-u) s}{72}+\frac{3 s}{16}-\frac{n^{2}(t-u)}{36} \\
& \left.-\frac{n(t-u)}{18}-\frac{(t-u)}{36}-\frac{1}{2 n}+\frac{1}{2 n^{2}}\right\} \\
+k_{1}(s) & \left\{\frac{n^{2} s^{3}}{576}+\frac{n s^{3}}{96}+\frac{11 s^{3}}{576}-\frac{17 n^{2} s^{2}}{288}-\frac{11 n s^{2}}{288}+\frac{1}{576} n^{2}(t-u) s^{2}-\frac{1}{144} n(t-u) s^{2}\right. \\
& -\frac{5(t-u) s^{2}}{576}-\frac{3 s^{2}}{32}+\frac{n^{2} s}{12}+\frac{n s}{144}-\frac{1}{96} n^{2}(t-u) s+\frac{n(t-u) s}{32}+\frac{(t-u) s}{48} \\
& -\frac{s}{8 n}+\frac{s}{8 n^{2}}+\frac{31 s}{144}+\frac{n}{6}-\frac{n^{2}(t-u)}{48}-\frac{n(t-u)}{24}+\frac{5(t-u)}{48} \\
& \left.-\frac{3}{4 n}+\frac{1}{n^{2}}+\frac{1}{24}\right\}
\end{aligned}
$$




$$
\begin{aligned}
& +\bar{J}(t)\left\{-\frac{5}{144} L n^{2} s^{3}-\frac{19 L s^{3}}{144}+L_{0}^{r} s^{3}+\frac{4 L_{1}^{r} s^{3}}{3}+\frac{8 L_{2}^{r} s^{3}}{3}+\frac{L_{3}^{r} s^{3}}{3}+\frac{1}{96} \operatorname{Lns}^{3}\right. \\
& +\frac{2}{3} L_{0}^{r} n s^{3}+\frac{4}{3} L_{3}^{r} n s^{3}+\frac{17}{576} n^{2} \pi_{16} s^{3}-\frac{1}{288} n \pi_{16} s^{3}+\frac{29 \pi_{16} s^{3}}{192}+\frac{7}{144} L n^{2} s^{2} \\
& +\frac{31 L s^{2}}{36}-\frac{8 L_{0}^{r} s^{2}}{3}-\frac{8 L_{1}^{r} s^{2}}{3}-\frac{28 L_{2}^{r} s^{2}}{3}+2 L_{3}^{r} s^{2}-4 L_{4}^{r} s^{2}-L_{5}^{r} s^{2}-\frac{13}{144} L_{n} s^{2} \\
& -\frac{4}{3} L_{0}^{r} n s^{2}-\frac{14}{3} L_{3}^{r} n s^{2}+L_{5}^{r} n s^{2}-\frac{35}{144} n^{2} \pi_{16} s^{2}+\frac{19}{288} n \pi_{16} s^{2}-\frac{55 \pi_{16} s^{2}}{72} \\
& +\frac{1}{48} L(t-u) s^{2}-\frac{1}{3} L_{0}^{r}(t-u) s^{2}+\frac{2}{3} L_{1}^{r}(t-u) s^{2}-\frac{1}{3} L_{2}^{r}(t-u) s^{2} \\
& +\frac{1}{6} L_{3}^{r}(t-u) s^{2}+\frac{1}{48} L n(t-u) s^{2}-\frac{1}{3} L_{0}^{r} n(t-u) s^{2}+\frac{1}{6} L_{3}^{r} n(t-u) s^{2} \\
& -\frac{1}{432} n^{2} \pi_{16}(t-u) s^{2}-\frac{47 n \pi_{16}(t-u) s^{2}}{1728}-\frac{43 \pi_{16}(t-u) s^{2}}{1728}-\frac{20 L_{0}^{r} s^{2}}{3 n} \\
& -\frac{20 L_{3}^{r} s^{2}}{3 n}-\frac{5}{36} L n^{2} s-\frac{97 L s}{72}+\frac{4 L_{0}^{r} s}{3}+\frac{16 L_{1}^{r} s}{3}+\frac{32 L_{2}^{r} s}{3}-8 L_{3}^{r} s+2 L_{5}^{r} s \\
& +16 L_{6}^{r} s+4 L_{8}^{r} s-\frac{17 L n s}{72}+\frac{8 L_{0}^{r} n s}{3}+\frac{16 L_{3}^{r} n s}{3}-4 L_{5}^{r} n s+8 L_{8}^{r} n s \\
& +\frac{5}{12} n^{2} \pi_{16} s+\frac{n \pi_{16} s}{24}-\frac{\pi_{16} s}{2 n}+\frac{\pi_{16} s}{2 n^{2}}+\frac{13 \pi_{16} s}{16}+\frac{1}{48} L n^{2}(t-u) s-\frac{L(t-u) s}{24} \\
& +\frac{4 L_{0}^{r}(t-u) s}{3}-\frac{8 L_{1}^{r}(t-u) s}{3}+\frac{4 L_{2}^{r}(t-u) s}{3}-\frac{2 L_{3}^{r}(t-u) s}{3}-\frac{4 L_{4}^{r}(t-u) s}{3} \\
& -\frac{L_{5}^{r}(t-u) s}{3}-\frac{1}{16} L n(t-u) s+\frac{4}{3} L_{0}^{r} n(t-u) s-\frac{2}{3} L_{3}^{r} n(t-u) s-\frac{1}{3} L_{5}^{r} n(t-u) s \\
& +\frac{17}{216} n^{2} \pi_{16}(t-u) s+\frac{253}{864} n \pi_{16}(t-u) s+\frac{155 \pi_{16}(t-u) s}{864}+\frac{L s}{4 n}+\frac{64 L_{0}^{r} s}{3 n} \\
& +\frac{64 L_{3}^{r} s}{3 n}-\frac{L s}{4 n^{2}}+\frac{19 L}{18}+\frac{16 L_{0}^{r}}{3}+\frac{32 L_{3}^{r}}{3}-8 L_{5}^{r}+16 L_{8}^{r}-\frac{5 L n}{18}+\frac{5 n \pi_{16}}{6} \\
& -\frac{4 \pi_{16}}{n}+\frac{5 \pi_{16}}{n^{2}}+\frac{2 \pi_{16}}{9}-\frac{1}{12} \operatorname{Ln}^{2}(t-u)-\frac{L(t-u)}{6}+\frac{16 L_{4}^{r}(t-u)}{3} \\
& +\frac{4 L_{5}^{r}(t-u)}{3}-\frac{L n(t-u)}{12}+\frac{4 L_{5}^{r} n(t-u)}{3}-\frac{11}{48} n^{2} \pi_{16}(t-u) \\
& -\frac{59 n \pi_{16}(t-u)}{144}+\frac{\pi_{16}(t-u)}{8 n}-\frac{\pi_{16}(t-u)}{8 n^{2}}+\frac{3 \pi_{16}(t-u)}{8}+\frac{5 L}{2 n}-\frac{80 L_{0}^{r}}{3 n} \\
& \left.-\frac{80 L_{3}^{r}}{3 n}+\frac{16 L_{5}^{r}}{n}-\frac{48 L_{8}^{r}}{n}-\frac{3 L}{n^{2}}\right\} \\
& B_{T}(t)=k_{3}(t)\left\{\frac{n t^{2}}{24}+\frac{t^{2}}{24}-\frac{n t}{9}-\frac{t}{6 n}+\frac{t}{6 n^{2}}+\frac{t}{18}+\frac{n}{18}+\frac{1}{3 n}-\frac{1}{3 n^{2}}-\frac{5}{18}\right\} \\
& +k_{2}(t)\left\{-\frac{3 t^{3}}{32}+\frac{9 t^{2}}{16}-\frac{9 t}{8}+\frac{3}{4}\right\} \\
& +k_{1}(t)\left\{-\frac{5 n t^{3}}{288}-\frac{11 t^{3}}{288}+\frac{n t^{2}}{16}+\frac{3 t^{2}}{16}-\frac{n t}{72}+\frac{t}{4 n}-\frac{t}{4 n^{2}}-\frac{31 t}{72}-\frac{n}{12}-\frac{1}{2 n}\right. \\
& \left.+\frac{1}{2 n^{2}}+\frac{5}{12}\right\}
\end{aligned}
$$




$$
\begin{aligned}
& +\bar{J}(t)\left\{\frac{19 L t^{3}}{72}-2 L_{0}^{r} t^{3}-\frac{8 L_{1}^{r} t^{3}}{3}-\frac{16 L_{2}^{r} t^{3}}{3}-\frac{2 L_{3}^{r} t^{3}}{3}+\frac{5}{144} L n t^{3}-\frac{17}{288} n \pi_{16} t^{3}\right. \\
& -\frac{29 \pi_{16} t^{3}}{96}-\frac{31 L t^{2}}{18}+12 L_{0}^{r} t^{2}+\frac{32 L_{1}^{r} t^{2}}{3}+\frac{88 L_{2}^{r} t^{2}}{3}+\frac{8 L_{3}^{r} t^{2}}{3}+8 L_{4}^{r} t^{2}+2 L_{5}^{r} t^{2} \\
& -\frac{11}{36} L n t^{2}+\frac{1}{18} n \pi_{16} t^{2}+\frac{55 \pi_{16} t^{2}}{36}+\frac{151 L t}{36}-24 L_{0}^{r} t-\frac{64 L_{1}^{r} t}{3}-\frac{176 L_{2}^{r} t}{3} \\
& -\frac{16 L_{3}^{r} t}{3}-16 L_{4}^{r} t-4 L_{5}^{r} t-32 L_{6}^{r} t-8 L_{8}^{r} t+\frac{31 L n t}{36}+\frac{29 n \pi_{16} t}{72}+\frac{\pi_{16} t}{n}-\frac{\pi_{16} t}{n^{2}} \\
& -\frac{27 \pi_{16} t}{8}-\frac{L t}{2 n}+\frac{L t}{2 n^{2}}-\frac{65 L}{18}+16 L_{0}^{r}+\frac{64 L_{1}^{r}}{3}+\frac{128 L_{2}^{r}}{3}+\frac{16 L_{3}^{r}}{3}+64 L_{6}^{r} \\
& \left.+16 L_{8}^{r}-\frac{7 L n}{9}-\frac{5 n \pi_{16}}{9}-\frac{2 \pi_{16}}{n}+\frac{2 \pi_{16}}{n^{2}}+\frac{55 \pi_{16}}{18}+\frac{L}{n}-\frac{L}{n^{2}}\right\} \\
& C_{S}(s)=k_{3}(s)\left\{\frac{n s^{2}}{24}+\frac{s^{2}}{24}-\frac{5 n s}{18}+\frac{s}{6 n}-\frac{7 s}{36}-\frac{1}{3 n^{2}}+\frac{2}{3 n^{3}}-\frac{1}{6}\right\} \\
& +k_{2}(s)\left\{\frac{3 n s^{3}}{32}-\frac{s^{3}}{32}+\frac{3 s^{2}}{16}+\frac{1}{2 n^{2}}-\frac{3}{4 n^{3}}\right\} \\
& +k_{1}(s)\left\{\frac{13 n s^{3}}{288}+\frac{s^{3}}{288}-\frac{23 n s^{2}}{144}-\frac{s^{2}}{72}+\frac{5 n s}{12}-\frac{s}{2 n}+\frac{s}{4}+\frac{1}{2 n^{2}}-\frac{1}{n^{3}}+\frac{1}{4}\right\} \\
& +\bar{J}(s)\left\{\frac{L s^{3}}{18}+\frac{4 L_{0}^{r} s^{3}}{3}+\frac{8 L_{3}^{r} s^{3}}{3}-\frac{5}{18} L n s^{3}+8 L_{1}^{r} n s^{3}+\frac{8}{3} L_{2}^{r} n s^{3}+\frac{85}{288} n \pi_{16} s^{3}\right. \\
& -\frac{19 \pi_{16} s^{3}}{288}-\frac{35 L s^{2}}{72}-\frac{8 L_{0}^{r} s^{2}}{3}+8 L_{1}^{r} s^{2}+\frac{8 L_{2}^{r} s^{2}}{3}-\frac{28 L_{3}^{r} s^{2}}{3}+2 L_{5}^{r} s^{2}+\frac{19}{72} L_{n} s^{2} \\
& -32 L_{1}^{r} n s^{2}-\frac{16}{3} L_{2}^{r} n s^{2}+16 L_{4}^{r} n s^{2}-\frac{7}{12} n \pi_{16} s^{2}+\frac{3 \pi_{16} s^{2}}{16}-\frac{8 L_{1}^{r} s^{2}}{n}-\frac{8 L_{2}^{r} s^{2}}{3 n} \\
& +\frac{20 L_{0}^{r} s^{2}}{3 n^{2}}+\frac{20 L_{3}^{r} s^{2}}{3 n^{2}}-\frac{L s}{9}+\frac{16 L_{0}^{r} s}{3}-32 L_{1}^{r} s-\frac{16 L_{2}^{r} s}{3}+\frac{32 L_{3}^{r} s}{3}+16 L_{4}^{r} s \\
& -8 L_{5}^{r} s+16 L_{8}^{r} s-\frac{11 L n s}{18}+32 L_{1}^{r} n s+\frac{32 L_{2}^{r} n s}{3}-32 L_{4}^{r} n s+32 L_{6}^{r} n s \\
& +\frac{35 n \pi_{16} s}{18}-\frac{7 \pi_{16} s}{4 n}+\frac{89 \pi_{16} s}{72}+\frac{L s}{n}+\frac{32 L_{1}^{r} s}{n}+\frac{16 L_{2}^{r} s}{3 n}-\frac{16 L_{4}^{r} s}{n}-\frac{64 L_{0}^{r} s}{3 n^{2}} \\
& -\frac{64 L_{3}^{r} s}{3 n^{2}}-\frac{L}{3}+32 L_{1}^{r}+\frac{32 L_{2}^{r}}{3}-32 L_{4}^{r}+32 L_{6}^{r}+\frac{3 \pi_{16}}{n^{2}}-\frac{11 \pi_{16}}{2 n^{3}}+\frac{10 \pi_{16}}{9} \\
& -\frac{L}{n}-\frac{32 L_{1}^{r}}{n}-\frac{32 L_{2}^{r}}{3 n}+\frac{32 L_{4}^{r}}{n}-\frac{32 L_{6}^{r}}{n}-\frac{2 L}{n^{2}}+\frac{80 L_{0}^{r}}{3 n^{2}}+\frac{80 L_{3}^{r}}{3 n^{2}}-\frac{16 L_{5}^{r}}{n^{2}} \\
& \left.+\frac{48 L_{8}^{r}}{n^{2}}+\frac{7 L}{2 n^{3}}\right\} \\
& C_{T}(t)=k_{3}(t)\left\{\frac{n t^{2}}{24}+\frac{t^{2}}{24}-\frac{n t}{9}-\frac{t}{6 n}-\frac{t}{9}+\frac{n}{18}+\frac{1}{3 n}+\frac{1}{18}\right\} \\
& +k_{2}(t)\left\{-\frac{t^{3}}{32}+\frac{3 t^{2}}{16}-\frac{3 t}{8}+\frac{1}{4}\right\} \\
& +k_{1}(t)\left\{-\frac{5 n t^{3}}{288}+\frac{t^{3}}{288}+\frac{n t^{2}}{16}-\frac{t^{2}}{16}-\frac{n t}{72}+\frac{t}{4 n}+\frac{11 t}{72}-\frac{n}{12}-\frac{1}{2 n}-\frac{1}{12}\right\}
\end{aligned}
$$




$$
\begin{aligned}
+\bar{J}(t) & \left\{\frac{L t^{3}}{18}-2 L_{0}^{r} t^{3}-\frac{2 L_{3}^{r} t^{3}}{3}+\frac{5}{144} L n t^{3}-\frac{17}{288} n \pi_{16} t^{3}-\frac{19 \pi_{16} t^{3}}{288}-\frac{7 L t^{2}}{18}+12 L_{0}^{r} t^{2}\right. \\
& +\frac{8 L_{3}^{r} t^{2}}{3}+2 L_{5}^{r} t^{2}-\frac{11}{36} L n t^{2}+\frac{1}{18} n \pi_{16} t^{2}+\frac{\pi_{16} t^{2}}{12}+\frac{37 L t}{36}-24 L_{0}^{r} t-\frac{16 L_{3}^{r} t}{3} \\
& -4 L_{5}^{r} t-8 L_{8}^{r} t+\frac{31 L n t}{36}+\frac{29 n \pi_{16} t}{72}+\frac{\pi_{16} t}{n}+\frac{13 \pi_{16} t}{72}-\frac{L t}{2 n}-\frac{17 L}{18}+16 L_{0}^{r} \\
& \left.+\frac{16 L_{3}^{r}}{3}+16 L_{8}^{r}-\frac{7 L n}{9}-\frac{5 n \pi_{16}}{9}-\frac{2 \pi_{16}}{n}-\frac{\pi_{16}}{6}+\frac{L}{n}\right\}
\end{aligned}
$$

\section{A.3 Pseudo-real or two-colour}

$$
\begin{aligned}
& B_{S}(s, t-u)=k_{4}(s)\left\{\frac{n^{2}}{12}+\frac{n}{12}-\frac{1}{6}+\frac{1}{2 n}+\frac{1}{2 n^{2}}\right\}(t-u) \\
& +k_{3}(s)\left\{\frac{s^{2} n^{2}}{48}-\frac{s n^{2}}{18}-\frac{1}{48} s(t-u) n^{2}+\frac{(t-u) n^{2}}{36}+\frac{s n}{24}+\frac{s(t-u) n}{24}\right. \\
& -\frac{(t-u) n}{72}+\frac{n}{9}-\frac{s^{2}}{48}+\frac{s}{18}-\frac{s(t-u)}{48}-\frac{7(t-u)}{72}-\frac{1}{36}-\frac{s}{12 n}+\frac{(t-u)}{12 n} \\
& \left.-\frac{1}{2 n}-\frac{s}{12 n^{2}}+\frac{(t-u)}{12 n^{2}}-\frac{2}{3 n^{2}}\right\} \\
& +k_{2}(s)\left\{\frac{n^{2} s^{3}}{64}+\frac{n s^{3}}{64}+\frac{3 s^{3}}{64}-\frac{3 n s^{2}}{32}-\frac{1}{576} n^{2}(t-u) s^{2}+\frac{1}{288} n(t-u) s^{2}\right. \\
& -\frac{(t-u) s^{2}}{576}-\frac{9 s^{2}}{32}+\frac{1}{72} n^{2}(t-u) s-\frac{n(t-u) s}{36}+\frac{(t-u) s}{72}+\frac{3 s}{16}-\frac{n^{2}(t-u)}{36} \\
& \left.+\frac{n(t-u)}{18}-\frac{(t-u)}{36}+\frac{1}{2 n}+\frac{1}{2 n^{2}}\right\} \\
& +k_{1}(s)\left\{\frac{n^{2} s^{3}}{576}-\frac{n s^{3}}{96}+\frac{11 s^{3}}{576}-\frac{17 n^{2} s^{2}}{288}+\frac{11 n s^{2}}{288}+\frac{1}{576} n^{2}(t-u) s^{2}\right. \\
& +\frac{1}{144} n(t-u) s^{2}-\frac{5(t-u) s^{2}}{576}-\frac{3 s^{2}}{32}+\frac{n^{2} s}{12}-\frac{n s}{144}-\frac{1}{96} n^{2}(t-u) s \\
& -\frac{n(t-u) s}{32}+\frac{(t-u) s}{48}+\frac{s}{8 n}+\frac{s}{8 n^{2}}+\frac{31 s}{144}-\frac{n}{6}-\frac{n^{2}(t-u)}{48} \\
& \left.+\frac{n(t-u)}{24}+\frac{5(t-u)}{48}+\frac{3}{4 n}+\frac{1}{n^{2}}+\frac{1}{24}\right\} \\
& +\bar{J}(s)\left\{-\frac{5}{144} L n^{2} s^{3}-\frac{19 L s^{3}}{144}-L_{0}^{r} s^{3}+\frac{4 L_{1}^{r} s^{3}}{3}+\frac{8 L_{2}^{r} s^{3}}{3}-\frac{L_{3}^{r} s^{3}}{3}-\frac{1}{96} \operatorname{Lns}^{3}\right. \\
& +\frac{2}{3} L_{0}^{r} n s^{3}+\frac{4}{3} L_{3}^{r} n s^{3}+\frac{17}{576} n^{2} \pi_{16} s^{3}+\frac{1}{288} n \pi_{16} s^{3}+\frac{29 \pi_{16} s^{3}}{192}+\frac{7}{144} L n^{2} s^{2} \\
& +\frac{31 L s^{2}}{36}+\frac{8 L_{0}^{r} s^{2}}{3}-\frac{8 L_{1}^{r} s^{2}}{3}-\frac{28 L_{2}^{r} s^{2}}{3}-2 L_{3}^{r} s^{2}-4 L_{4}^{r} s^{2}+L_{5}^{r} s^{2}+\frac{13}{144} L_{n} s^{2} \\
& -\frac{4}{3} L_{0}^{r} n s^{2}-\frac{14}{3} L_{3}^{r} n s^{2}+L_{5}^{r} n s^{2}-\frac{35}{144} n^{2} \pi_{16} s^{2}-\frac{19}{288} n \pi_{16} s^{2}-\frac{55 \pi_{16} s^{2}}{72} \\
& +\frac{1}{48} L(t-u) s^{2}+\frac{1}{3} L_{0}^{r}(t-u) s^{2}+\frac{2}{3} L_{1}^{r}(t-u) s^{2}-\frac{1}{3} L_{2}^{r}(t-u) s^{2}-\frac{1}{6} L_{3}^{r}(t-u) s^{2}
\end{aligned}
$$




$$
\begin{aligned}
& -\frac{1}{48} L n(t-u) s^{2}-\frac{1}{3} L_{0}^{r} n(t-u) s^{2}+\frac{1}{6} L_{3}^{r} n(t-u) s^{2}-\frac{1}{432} n^{2} \pi_{16}(t-u) s^{2} \\
& +\frac{47 n \pi_{16}(t-u) s^{2}}{1728}-\frac{43 \pi_{16}(t-u) s^{2}}{1728}-\frac{20 L_{0}^{r} s^{2}}{3 n}-\frac{20 L_{3}^{r} s^{2}}{3 n}-\frac{5}{36} L n^{2} s-\frac{97 L s}{72} \\
& -\frac{4 L_{0}^{r} s}{3}+\frac{16 L_{1}^{r} s}{3}+\frac{32 L_{2}^{r} s}{3}+8 L_{3}^{r} s-2 L_{5}^{r} s+16 L_{6}^{r} s-4 L_{8}^{r} s+\frac{17 L n s}{72}+\frac{8 L_{0}^{r} n s}{3} \\
& +\frac{16 L_{3}^{r} n s}{3}-4 L_{5}^{r} n s+8 L_{8}^{r} n s+\frac{5}{12} n^{2} \pi_{16} s-\frac{n \pi_{16} s}{24}+\frac{\pi_{16} s}{2 n}+\frac{\pi_{16} s}{2 n^{2}}+\frac{13 \pi_{16} s}{16} \\
& +\frac{1}{48} L n^{2}(t-u) s-\frac{L(t-u) s}{24}-\frac{4 L_{0}^{r}(t-u) s}{3}-\frac{8 L_{1}^{r}(t-u) s}{3}+\frac{4 L_{2}^{r}(t-u) s}{3} \\
& +\frac{2 L_{3}^{r}(t-u) s}{3}-\frac{4 L_{4}^{r}(t-u) s}{3}+\frac{L_{5}^{r}(t-u) s}{3}+\frac{1}{16} L n(t-u) s+\frac{4}{3} L_{0}^{r} n(t-u) s \\
& -\frac{2}{3} L_{3}^{r} n(t-u) s-\frac{1}{3} L_{5}^{r} n(t-u) s+\frac{17}{216} n^{2} \pi_{16}(t-u) s-\frac{253}{864} n \pi_{16}(t-u) s \\
& +\frac{155 \pi_{16}(t-u) s}{864}-\frac{L s}{4 n}+\frac{64 L_{0}^{r} s}{3 n}+\frac{64 L_{3}^{r} s}{3 n}-\frac{L s}{4 n^{2}}+\frac{19 L}{18}-\frac{16 L_{0}^{r}}{3}-\frac{32 L_{3}^{r}}{3}+8 L_{5}^{r} \\
& -16 L_{8}^{r}+\frac{5 L n}{18}-\frac{5 n \pi_{16}}{6}+\frac{4 \pi_{16}}{n}+\frac{5 \pi_{16}}{n^{2}}+\frac{2 \pi_{16}}{9}-\frac{1}{12} L n^{2}(t-u)-\frac{L(t-u)}{6} \\
& +\frac{16 L_{4}^{r}(t-u)}{3}-\frac{4 L_{5}^{r}(t-u)}{3}+\frac{\operatorname{Ln}(t-u)}{12}+\frac{4 L_{5}^{r} n(t-u)}{3}-\frac{11}{48} n^{2} \pi_{16}(t-u) \\
& +\frac{59 n \pi_{16}(t-u)}{144}-\frac{\pi_{16}(t-u)}{8 n}-\frac{\pi_{16}(t-u)}{8 n^{2}}+\frac{3 \pi_{16}(t-u)}{8}-\frac{5 L}{2 n}-\frac{80 L_{0}^{r}}{3 n}-\frac{80 L_{3}^{r}}{3 n} \\
& \left.+\frac{16 L_{5}^{r}}{n}-\frac{48 L_{8}^{r}}{n}-\frac{3 L}{n^{2}}\right\} \\
& B_{T}(t)=k_{3}(t)\left\{-\frac{n t^{2}}{24}+\frac{t^{2}}{24}+\frac{n t}{9}+\frac{t}{6 n}+\frac{t}{6 n^{2}}+\frac{t}{18}-\frac{n}{18}-\frac{1}{3 n}-\frac{1}{3 n^{2}}-\frac{5}{18}\right\} \\
& +k_{2}(t)\left\{-\frac{3 t^{3}}{32}+\frac{9 t^{2}}{16}-\frac{9 t}{8}+\frac{3}{4}\right\} \\
& +k_{1}(t)\left\{\frac{5 n t^{3}}{288}-\frac{11 t^{3}}{288}-\frac{n t^{2}}{16}+\frac{3 t^{2}}{16}+\frac{n t}{72}-\frac{t}{4 n}-\frac{t}{4 n^{2}}-\frac{31 t}{72}+\frac{n}{12}\right. \\
& \left.+\frac{1}{2 n}+\frac{1}{2 n^{2}}+\frac{5}{12}\right\} \\
& +\bar{J}(t)\left\{\frac{19 L t^{3}}{72}+2 L_{0}^{r} t^{3}-\frac{8 L_{1}^{r} t^{3}}{3}-\frac{16 L_{2}^{r} t^{3}}{3}+\frac{2 L_{3}^{r} t^{3}}{3}-\frac{5}{144} L n t^{3}+\frac{17}{288} n \pi_{16} t^{3}\right. \\
& -\frac{29 \pi_{16} t^{3}}{96}-\frac{31 L t^{2}}{18}-12 L_{0}^{r} t^{2}+\frac{32 L_{1}^{r} t^{2}}{3}+\frac{88 L_{2}^{r} t^{2}}{3}-\frac{8 L_{3}^{r} t^{2}}{3}+8 L_{4}^{r} t^{2}-2 L_{5}^{r} t^{2} \\
& +\frac{11}{36} L n t^{2}-\frac{1}{18} n \pi_{16} t^{2}+\frac{55 \pi_{16} t^{2}}{36}+\frac{151 L t}{36}+24 L_{0}^{r} t-\frac{64 L_{1}^{r} t}{3}-\frac{176 L_{2}^{r} t}{3}+\frac{16 L_{3}^{r} t}{3} \\
& -16 L_{4}^{r} t+4 L_{5}^{r} t-32 L_{6}^{r} t+8 L_{8}^{r} t-\frac{31 L n t}{36}-\frac{29 n \pi_{16} t}{72}-\frac{\pi_{16} t}{n}-\frac{\pi_{16} t}{n^{2}}-\frac{27 \pi_{16} t}{8} \\
& +\frac{L t}{2 n}+\frac{L t}{2 n^{2}}-\frac{65 L}{18}-16 L_{0}^{r}+\frac{64 L_{1}^{r}}{3}+\frac{128 L_{2}^{r}}{3}-\frac{16 L_{3}^{r}}{3}+64 L_{6}^{r}-16 L_{8}^{r}+\frac{7 L n}{9} \\
& \left.+\frac{5 n \pi_{16}}{9}+\frac{2 \pi_{16}}{n}+\frac{2 \pi_{16}}{n^{2}}+\frac{55 \pi_{16}}{18}-\frac{L}{n}-\frac{L}{n^{2}}\right\}
\end{aligned}
$$




$$
\begin{aligned}
C_{S}(s)= & k_{3}(s)\left\{\frac{n s^{2}}{24}-\frac{s^{2}}{24}-\frac{5 n s}{18}+\frac{s}{6 n}+\frac{7 s}{36}+\frac{1}{3 n^{2}}+\frac{2}{3 n^{3}}+\frac{1}{6}\right\} \\
+k_{2}(s) & \left\{\frac{3 n s^{3}}{32}+\frac{s^{3}}{32}-\frac{3 s^{2}}{16}-\frac{1}{2 n^{2}}-\frac{3}{4 n^{3}}\right\} \\
+k_{1}(s) & \left\{\frac{13 n s^{3}}{288}-\frac{s^{3}}{288}-\frac{23 n s^{2}}{144}+\frac{s^{2}}{72}+\frac{5 n s}{12}-\frac{s}{2 n}-\frac{s}{4}-\frac{1}{2 n^{2}}-\frac{1}{n^{3}}-\frac{1}{4}\right\} \\
+\bar{J}(s) & \left\{-\frac{L s^{3}}{18}+\frac{4 L_{0}^{r} s^{3}}{3}+\frac{8 L_{3}^{r} s^{3}}{3}-\frac{5}{18} L n s^{3}+8 L_{1}^{r} n s^{3}+\frac{8}{3} L_{2}^{r} n s^{3}+\frac{85}{288} n \pi_{16} s^{3}\right. \\
+ & \frac{19 \pi_{16} s^{3}}{288}+\frac{35 L s^{2}}{72}-\frac{8 L_{0}^{r} s^{2}}{3}-8 L_{1}^{r} s^{2}-\frac{8 L_{2}^{r} s^{2}}{3}-\frac{28 L_{3}^{r} s^{2}}{3}+2 L_{5}^{r} s^{2}+\frac{19}{72} L n s^{2} \\
& -32 L_{1}^{r} n s^{2}-\frac{16}{3} L_{2}^{r} n s^{2}+16 L_{4}^{r} n s^{2}-\frac{7}{12} n \pi_{16} s^{2}-\frac{3 \pi_{16} s^{2}}{16}-\frac{8 L_{1}^{r} s^{2}}{n}-\frac{8 L_{2}^{r} s^{2}}{3 n} \\
+ & \frac{20 L_{0}^{r} s^{2}}{3 n^{2}}+\frac{20 L_{3}^{r} s^{2}}{3 n^{2}}+\frac{L s}{9}+\frac{16 L_{0}^{r} s}{3}+32 L_{1}^{r} s+\frac{16 L_{2}^{r} s}{3}+\frac{32 L_{3}^{r} s}{3}-16 L_{4}^{r} s-8 L_{5}^{r} s \\
+ & 16 L_{8}^{r} s-\frac{11 L n s}{18}+32 L_{1}^{r} n s+\frac{32 L_{2}^{r} n s}{3}-32 L_{4}^{r} n s+32 L_{6}^{r} n s+\frac{35 n \pi_{16} s}{18} \\
& -\frac{7 \pi_{16} s}{4 n}-\frac{89 \pi_{16} s}{72}+\frac{L s}{n}+\frac{32 L_{1}^{r} s}{n}+\frac{16 L_{2}^{r} s}{3 n}-\frac{16 L_{4}^{r} s}{n}-\frac{64 L_{0}^{r} s}{3 n^{2}}-\frac{64 L_{3}^{r} s}{3 n^{2}}+\frac{L}{3}-32 L_{1}^{r} \\
& -\frac{32 L_{2}^{r}}{3}+32 L_{4}^{r}-32 L_{6}^{r}-\frac{3 \pi_{16}}{n^{2}}-\frac{11 \pi_{16}}{2 n^{3}}-\frac{10 \pi_{16}}{9}-\frac{L}{n}-\frac{32 L_{1}^{r}}{n}-\frac{32 L_{2}^{r}}{3 n} \\
+ & \left.\frac{32 L_{4}^{r}}{n}-\frac{32 L_{6}^{r}}{n}+\frac{2 L}{n^{2}}+\frac{80 L_{0}^{r}}{3 n^{2}}+\frac{80 L_{3}^{r}}{3 n^{2}}-\frac{16 L_{5}^{r}}{n^{2}}+\frac{48 L_{8}^{r}}{n^{2}}+\frac{7 L}{2 n^{3}}\right\}
\end{aligned}
$$$$
C_{T}(t)=k_{3}(t)\left\{\frac{n t^{2}}{24}-\frac{t^{2}}{24}-\frac{n t}{9}-\frac{t}{6 n}+\frac{t}{9}+\frac{n}{18}+\frac{1}{3 n}-\frac{1}{18}\right\}
$$$$
+k_{2}(t)\left\{\frac{t^{3}}{32}-\frac{3 t^{2}}{16}+\frac{3 t}{8}-\frac{1}{4}\right\}
$$$$
+k_{1}(t)\left\{-\frac{5 n t^{3}}{288}-\frac{t^{3}}{288}+\frac{n t^{2}}{16}+\frac{t^{2}}{16}-\frac{n t}{72}+\frac{t}{4 n}-\frac{11 t}{72}-\frac{n}{12}-\frac{1}{2 n}+\frac{1}{12}\right\}
$$$$
+\bar{J}(t)\left\{-\frac{L t^{3}}{18}-2 L_{0}^{r} t^{3}-\frac{2 L_{3}^{r} t^{3}}{3}+\frac{5}{144} L n t^{3}-\frac{17}{288} n \pi_{16} t^{3}+\frac{19 \pi_{16} t^{3}}{288}+\frac{7 L t^{2}}{18}\right.
$$$$
+12 L_{0}^{r} t^{2}+\frac{8 L_{3}^{r} t^{2}}{3}+2 L_{5}^{r} t^{2}-\frac{11}{36} L n t^{2}+\frac{1}{18} n \pi_{16} t^{2}-\frac{\pi_{16} t^{2}}{12}-\frac{37 L t}{36}-24 L_{0}^{r} t
$$$$
-\frac{16 L_{3}^{r} t}{3}-4 L_{5}^{r} t-8 L_{8}^{r} t+\frac{31 L n t}{36}+\frac{29 n \pi_{16} t}{72}+\frac{\pi_{16} t}{n}-\frac{13 \pi_{16} t}{72}-\frac{L t}{2 n}
$$$$
\left.+\frac{17 L}{18}+16 L_{0}^{r}+\frac{16 L_{3}^{r}}{3}+16 L_{8}^{r}-\frac{7 L n}{9}-\frac{5 n \pi_{16}}{9}-\frac{2 \pi_{16}}{n}+\frac{\pi_{16}}{6}+\frac{L}{n}\right\}
$$

\section{B Polynomial parts}

Divergent parts can be put here 


\section{B.1 Complex or QCD}

The coefficients of polynomials part for $B_{P}$ at NNLO.

$$
\begin{aligned}
& \gamma_{1}=32 K_{13}^{r}+32 K_{14}^{r} n-96 K_{17}^{r}-96 K_{18}^{r} n+96 K_{25}^{r}+32 K_{26}^{r} n+64 K_{3}^{r}-64 K_{37}^{r} \\
& +96 K_{39}^{r}+32 K_{40}^{r} n+\frac{29 L^{2} n^{2}}{36}+\frac{19 L^{2}}{n^{2}}+\frac{L^{2}}{3}-\frac{80 L L_{0}^{r} n}{3}+\frac{64 L L_{0}^{r}}{3 n}-64 L L_{1}^{r} \\
& -\frac{224 L L_{2}^{r}}{3}-8 L L_{3}^{r} n+\frac{64 L L_{3}^{r}}{3 n}+\frac{64 L L_{4}^{r}}{3}+\frac{40 L L_{5}^{r} n}{3}-\frac{96 L L_{5}^{r}}{n}-160 L L_{6}^{r} \\
& -32 L L_{7}^{r}-64 L L_{8}^{r} n+\frac{224 L L_{8}^{r}}{n}+256 L_{4}^{r} L_{8}^{r} n+256 L_{5}^{r} L_{8}^{r}-512 L_{6}^{r} L_{8}^{r} n-512\left(L_{8}^{r}\right)^{2} \\
& +\pi_{16}^{2}\left(\frac{n^{2} \pi^{2}}{27}+\frac{1645 n^{2}}{1728}-\frac{35}{2 n^{2}}+\frac{4 \pi^{2}}{9}-\frac{181}{54}\right) \\
& +\pi_{16}\left(\frac{229 L n^{2}}{216}+\frac{4 L}{n^{2}}-\frac{26 L}{9}-\frac{80 L_{0}^{r} n}{9}+\frac{256 L_{0}^{r}}{9 n}-\frac{32 L_{1}^{r}}{3}-\frac{368 L_{2}^{r}}{9}\right. \\
& \left.-\frac{8 L_{3}^{r} n}{3}+\frac{256 L_{3}^{r}}{9 n}+\frac{256 L_{4}^{r}}{9}+\frac{64 L_{5}^{r} n}{9}-\frac{64 L_{5}^{r}}{n}-128 L_{6}^{r}-32 L_{8}^{r} n+\frac{192 L_{8}^{r}}{n}\right) \\
& \gamma_{2}=-32 K_{13}^{r}-32 K_{14}^{r} n+64 K_{17}^{r}+64 K_{18}^{r} n-16 K_{19}^{r}-8 K_{20}^{r} n-16 K_{23}^{r}-32 K_{28}^{r} \\
& -96 K_{3}^{r}-16 K_{33}^{r}+32 K_{37}^{r}-\frac{17}{36} L^{2} n^{2}-\frac{3 L^{2}}{2 n^{2}}-\frac{13 L^{2}}{3}+24 L L_{0}^{r} n+\frac{16 L L_{0}^{r}}{n} \\
& +\frac{176 L L_{1}^{r}}{3}+\frac{248 L L_{2}^{r}}{3}+\frac{4 L L_{3}^{r} n}{3}+\frac{16 L L_{3}^{r}}{n}+\frac{32 L L_{4}^{r}}{3}+\frac{20 L L_{5}^{r} n}{3}+48 L L_{6}^{r} \\
& +8 L L_{8}^{r} n-32 L_{4}^{r} L_{5}^{r} n-32\left(L_{5}^{r}\right)^{2}+64 L_{5}^{r} L_{6}^{r} n+64 L_{5}^{r} L_{8}^{r} \\
& +\pi_{16}\left(-\frac{445 L n^{2}}{432}-\frac{37 L}{36}+8 L_{0}^{r} n-\frac{16 L_{0}^{r}}{n}+\frac{80 L_{1}^{r}}{9}+\frac{512 L_{2}^{r}}{9}+\frac{16 L_{3}^{r} n}{9}\right. \\
& \left.-\frac{16 L_{3}^{r}}{n}+\frac{32 L_{4}^{r}}{9}+\frac{8 L_{5}^{r} n}{9}+48 L_{6}^{r}+8 L_{8}^{r} n\right) \\
& +\pi_{16}^{2}\left(-\frac{5}{72} n^{2} \pi^{2}-\frac{3865 n^{2}}{10368}+\frac{3}{n^{2}}-\frac{13 \pi^{2}}{36}-\frac{25}{432}\right) \\
& \gamma_{3}=2 K_{11}^{r}+8 K_{13}^{r}+8 K_{14}^{r} n-16 K_{17}^{r}-12 K_{18}^{r} n+16 K_{28}^{r}+48 K_{3}^{r}-4 K_{31}^{r}+8 K_{5}^{r} \\
& +2 K_{7}^{r}+2 K_{8}^{r} n+\frac{29 L^{2} n^{2}}{288}+\frac{27 L^{2}}{16}-8 L L_{0}^{r} n+\frac{20 L L_{0}^{r}}{3 n}-\frac{56 L L_{1}^{r}}{3}-40 L L_{2}^{r} \\
& -\frac{8 L L_{3}^{r} n}{3}+\frac{20 L L_{3}^{r}}{3 n}-8 L L_{4}^{r}-L L_{5}^{r} n \\
& +\pi_{16}\left(\frac{49 L n^{2}}{216}+\frac{29 L}{16}-\frac{7 L_{0}^{r} n}{3}+\frac{56 L_{0}^{r}}{9 n}-\frac{62 L_{1}^{r}}{9}-31 L_{2}^{r}-\frac{25 L_{3}^{r} n}{18}\right. \\
& \left.+\frac{56 L_{3}^{r}}{9 n}-\frac{20 L_{4}^{r}}{3}-\frac{2 L_{5}^{r} n}{3}\right) \\
& +\pi_{16}^{2}\left(\frac{n^{2} \pi^{2}}{72}+\frac{445 n^{2}}{5184}+\frac{3 \pi^{2}}{16}-\frac{23}{192}\right) \\
& \gamma_{4}=2 K_{11}^{r}+4 K_{18}^{r} n+4 K_{31}^{r}+8 K_{5}^{r}+2 K_{7}^{r}+2 K_{8}^{r} n+\frac{11 L^{2} n^{2}}{288}-\frac{7 L^{2}}{48}-\frac{4 L L_{0}^{r} n}{3} \\
& +\frac{20 L L_{0}^{r}}{3 n}-\frac{4 L L_{2}^{r}}{3}-2 L L_{3}^{r} n+\frac{20 L L_{3}^{r}}{3 n}+\frac{8 L L_{4}^{r}}{3}-\frac{L L_{5}^{r} n}{3}
\end{aligned}
$$




$$
\begin{aligned}
& +\pi_{16}\left(\frac{29 L n^{2}}{216}+\frac{17 L}{144}-\frac{13 L_{0}^{r} n}{9}+\frac{56 L_{0}^{r}}{9 n}-\frac{10 L_{1}^{r}}{3}-\frac{31 L_{2}^{r}}{9}-\frac{11 L_{3}^{r} n}{6}\right. \\
& \left.+\frac{56 L_{3}^{r}}{9 n}+\frac{20 L_{4}^{r}}{9}-\frac{4 L_{5}^{r} n}{9}\right) \\
& +\pi_{16}^{2}\left(-\frac{1}{108} n^{2} \pi^{2}+\frac{421 n^{2}}{3456}-\frac{\pi^{2}}{144}-\frac{115}{1728}\right) \\
\gamma_{5}= & K_{1}^{r}-8 K_{3}^{r}-4 K_{5}^{r}-\frac{5 L^{2} n^{2}}{1152}-\frac{15 L^{2}}{64}+\frac{5 L L_{0}^{r} n}{12}+\frac{5 L L_{1}^{r}}{2}+\frac{25 L L_{2}^{r}}{4}+\frac{5 L L_{3}^{r} n}{24} \\
& +\pi_{16}\left(-\frac{19 L n^{2}}{2304}-\frac{13 L}{32}+\frac{2 L_{0}^{r} n}{9}+\frac{7 L_{1}^{r}}{3}+\frac{35 L_{2}^{r}}{6}+\frac{5 L_{3}^{r} n}{18}\right) \\
& +\pi_{16}^{2}\left(\frac{n^{2} \pi^{2}}{3456}-\frac{1015 n^{2}}{165888}-\frac{\pi^{2}}{32}+\frac{23}{384}\right) \\
\gamma_{6}= & 3 K_{1}^{r}-4 K_{5}^{r}-\frac{5}{384} L^{2} n^{2}-\frac{5 L^{2}}{64}+\frac{7 L L_{0}^{r} n}{12}+\frac{5 L L_{1}^{r}}{6}+\frac{25 L L_{2}^{r}}{12}+\frac{23 L L_{3}^{r} n}{24} \\
& +\pi_{16}\left(-\frac{203 L n^{2}}{6912}-\frac{13 L}{96}+\frac{4 L_{0}^{r} n}{9}+\frac{7 L_{1}^{r}}{9}+\frac{35 L_{2}^{r}}{18}+\frac{17 L_{3}^{r} n}{18}\right) \\
& +\pi_{16}^{2}\left(-\frac{n^{2} \pi^{2}}{3456}-\frac{1933 n^{2}}{165888}-\frac{\pi^{2}}{96}+\frac{23}{1152}\right)
\end{aligned}
$$

The coefficients of polynomials part for $C_{P}$ at NNLO.

$$
\begin{aligned}
\delta_{1}= & 64 K_{10}^{r} n-128 K_{18}^{r}+128 K_{2}^{r}-64 K_{20}^{r}-128 K_{21}^{r}-128 K_{22}^{r} n+128 K_{26}^{r}+192 K_{27}^{r} n \\
& -128 K_{35}^{r}+128 K_{40}^{r}+64 K_{9}^{r}-\frac{14 L^{2}}{n^{3}}+\frac{2 L^{2}}{n}-\frac{192 L L_{0}^{r}}{n^{2}}-64 L L_{1}^{r} n+\frac{128 L L_{1}^{r}}{n} \\
& +\frac{32 L L_{2}^{r}}{n}-\frac{192 L L_{3}^{r}}{n^{2}}+64 L L_{4}^{r} n-\frac{96 L L_{4}^{r}}{n}+\frac{96 L L_{5}^{r}}{n^{2}}+16 L L_{5}^{r}-64 L L_{6}^{r} n+\frac{64 L L_{6}^{r}}{n} \\
& +\frac{64 L L_{7}^{r}}{n}-\frac{192 L L_{8}^{r}}{n^{2}}-32 L L_{8}^{r}-256\left(L_{4}^{r}\right)^{2} n-256 L_{4}^{r} L_{5}^{r}+1024 L_{4}^{r} L_{6}^{r} n+512 L_{4}^{r} L_{8}^{r} \\
& +512 L_{5}^{r} L_{6}^{r}-1024\left(L_{6}^{r}\right)^{2} n-1024 L_{6}^{r} L_{8}^{r} \\
& +\pi_{16}\left(-\frac{12 L}{n^{3}}+\frac{4 L}{n}-\frac{64 L_{0}^{r}}{n^{2}}+\frac{64 L_{1}^{r}}{n}-\frac{64 L_{3}^{r}}{n^{2}}-\frac{64 L_{4}^{r}}{n}+\frac{64 L_{5}^{r}}{n^{2}}+\frac{64 L_{6}^{r}}{n}-\frac{192 L_{8}^{r}}{n^{2}}\right) \\
& +\pi_{16}^{2}\left(\frac{10}{n^{3}}+\frac{3}{n}\right) \\
\delta_{2}= & -64 K_{10}^{r} n+128 K_{18}^{r}-192 K_{2}^{r}+32 K_{20}^{r}+64 K_{21}^{r}+64 K_{22}^{r} n-32 K_{32}^{r}+64 K_{35}^{r} \\
& -32 K_{38}^{r}-64 K_{9}^{r}+\frac{37 L^{2} n}{36}-\frac{3 L^{2}}{n}+\frac{416 L L_{0}^{r}}{3 n^{2}}-16 L L_{0}^{r}-\frac{96 L L_{1}^{r}}{n}-16 L L_{2}^{r} n \\
& -\frac{64 L L_{2}^{r}}{3 n}+\frac{416 L L_{3}^{r}}{3 n^{2}}-\frac{128 L L_{3}^{r}}{3}+16 L L_{4}^{r} n+\frac{32 L L_{4}^{r}}{n}+24 L L_{5}^{r}-32 L L_{6}^{r} n \\
& -48 L L_{8}^{r}+128\left(L_{4}^{r}\right)^{2} n+128 L_{4}^{r} L_{5}^{r}-256 L_{4}^{r} L_{6}^{r} n-256 L_{4}^{r} L_{8}^{r} \\
& +\pi_{16}\left(-\frac{31 L n}{12}-\frac{L}{n}+\frac{608 L_{0}^{r}}{9 n^{2}}-32 L_{1}^{r} n-\frac{64 L_{1}^{r}}{n}-\frac{16 L_{2}^{r}}{9 n}+\frac{608 L_{3}^{r}}{9 n^{2}}-\frac{176 L_{3}^{r}}{9}\right. \\
& \left.+32 L_{4}^{r} n+\frac{32 L_{4}^{r}}{n}+24 L_{5}^{r}-32 L_{6}^{r} n-48 L_{8}^{r}\right)
\end{aligned}
$$




$$
\begin{aligned}
& +\pi_{16}^{2}\left(-\frac{2 n \pi^{2}}{27}-\frac{373 n}{1296}-\frac{\pi^{2}}{3 n}+\frac{25}{4 n}\right) \\
\delta_{3}= & 16 K_{10}^{r} n+4 K_{15}^{r}+4 K_{16}^{r} n-32 K_{18}^{r}+96 K_{2}^{r}-8 K_{29}^{r}+16 K_{32}^{r}+16 K_{9}^{r}-\frac{13 L^{2} n}{36} \\
& -\frac{80 L L_{0}^{r}}{3 n^{2}}+\frac{20 L L_{0}^{r}}{3}+32 L L_{1}^{r} n+\frac{16 L L_{1}^{r}}{n}+\frac{20 L L_{2}^{r} n}{3}+\frac{16 L L_{2}^{r}}{3 n}-\frac{80 L L_{3}^{r}}{3 n^{2}}+\frac{88 L L_{3}^{r}}{3} \\
& -16 L L_{4}^{r} n-6 L L_{5}^{r} \\
& +\pi_{16}\left(\frac{11 L n}{12}-\frac{224 L_{0}^{r}}{9 n^{2}}+\frac{8 L_{0}^{r}}{9}+32 L_{1}^{r} n+\frac{16 L_{1}^{r}}{n}+\frac{8 L_{2}^{r} n}{9}+\frac{40 L_{2}^{r}}{9 n}\right. \\
& \left.-\frac{224 L_{3}^{r}}{9 n^{2}}+\frac{166 L_{3}^{r}}{9}-16 L_{4}^{r} n-6 L_{5}^{r}\right) \\
& +\pi_{16}^{2}\left(\frac{625 n}{1296}-\frac{25 n \pi^{2}}{432}\right) \\
\delta_{4}= & 4 K_{15}^{r}+4 K_{16}^{r} n+8 K_{29}^{r}+\frac{5 L^{2} n}{24}-4 L L_{0}^{r}-4 L L_{2}^{r} n-2 L L_{5}^{r} \\
& +\pi_{16}\left(\frac{L n}{8}+2 L_{3}^{r}-2 L_{5}^{r}\right)+\pi_{16}^{2}\left(\frac{n \pi^{2}}{144}+\frac{7 n}{32}\right) \\
\delta_{5}= & -16 K_{2}^{r}+2 K_{4}^{r}+2 K_{6}^{r}+\frac{55 L^{2} n}{192}-\frac{11 L L_{0}^{r}}{3}-8 L L_{1}^{r} n-\frac{8 L L_{2}^{r} n}{3}-\frac{17 L L_{3}^{r}}{3} \\
& +\pi_{16}\left(\frac{101 L n}{192}-\frac{29 L_{0}^{r}}{9}-8 L_{1}^{r} n-\frac{20 L_{2}^{r} n}{9}-\frac{97 L_{3}^{r}}{18}\right)+\pi_{16}^{2}\left(\frac{19 n \pi^{2}}{576}-\frac{115 n}{6912}\right) \\
\delta_{6}= & 6 K_{4}^{r}-2 K_{6}^{r}+\frac{5 L^{2} n}{192}-3 L L_{0}^{r}-L L_{3}^{r} \\
& +\pi_{16}\left(\frac{L n}{64}-3 L_{0}^{r}-\frac{5 L_{3}^{r}}{6}\right)+\pi_{16}^{2}\left(\frac{5 n \pi^{2}}{576}-\frac{437 n}{6912}\right) \\
&
\end{aligned}
$$

\section{B.2 Real or adjoint}

The coefficients of polynomials part for $B_{P}$ at NNLO.

$$
\begin{aligned}
\gamma_{1}= & 32 K_{13}^{r}+64 K_{14}^{r} n-96 K_{17}^{r}-192 K_{18}^{r} n+96 K_{25}^{r}+64 K_{26}^{r} n+64 K_{3}^{r}-64 K_{37}^{r} \\
& +96 K_{39}^{r}+64 K_{40}^{r} n+\frac{29 L^{2} n^{2}}{36}+\frac{19 L^{2}}{4 n^{2}}+\frac{83 L^{2} n}{36}-\frac{17 L^{2}}{4 n}+\frac{19 L^{2}}{12}-\frac{80 L L_{0}^{r} n}{3} \\
& +\frac{32 L L_{0}^{r}}{3 n}-\frac{80 L L_{0}^{r}}{3}-64 L L_{1}^{r}-\frac{224 L L_{2}^{r}}{3}-8 L L_{3}^{r} n+\frac{32 L L_{3}^{r}}{3 n}-\frac{56 L L_{3}^{r}}{3}+\frac{64 L L_{4}^{r}}{3} \\
& +\frac{40 L L_{5}^{r} n}{3}-\frac{48 L L_{5}^{r}}{n}+\frac{64 L L_{5}^{r}}{3}-160 L L_{6}^{r}-32 L L_{7}^{r}-64 L L_{8}^{r} n+\frac{112 L L_{8}^{r}}{n}-80 L L_{8}^{r} \\
& +512 L_{4}^{r} L_{8}^{r} n+256 L_{5}^{r} L_{8}^{r}-1024 L_{6}^{r} L_{8}^{r} n-512\left(L_{8}^{r}\right)^{2} \\
& +\pi_{16}\left(\frac{229 L n^{2}}{216}+\frac{L}{n^{2}}+\frac{623 L n}{216}-\frac{L}{n}+\frac{155 L}{108}-\frac{80 L_{0}^{r} n}{9}+\frac{128 L_{0}^{r}}{9 n}-\frac{224 L_{0}^{r}}{9}\right. \\
& -\frac{32 L_{1}^{r}}{3}-\frac{368 L_{2}^{r}}{9}-\frac{8 L_{3}^{r} n}{3}+\frac{128 L_{3}^{r}}{9 n}-\frac{56 L_{3}^{r}}{9}+\frac{256 L_{4}^{r}}{9}+\frac{64 L_{5}^{r} n}{9}-\frac{32 L_{5}^{r}}{n} \\
& \left.+\frac{136 L_{5}^{r}}{9}-128 L_{6}^{r}-32 L_{8}^{r} n+\frac{96 L_{8}^{r}}{n}-64 L_{8}^{r}\right)
\end{aligned}
$$




$$
\begin{aligned}
& +\pi_{16}^{2}\left(\frac{n^{2} \pi^{2}}{27}+\frac{1645 n^{2}}{1728}-\frac{35}{8 n^{2}}+\frac{10763 n}{5184}+\frac{27}{8 n}+\frac{13 \pi^{2}}{54}-\frac{2149}{1296}\right) \\
& \gamma_{2}=-32 K_{13}^{r}-64 K_{14}^{r} n+64 K_{17}^{r}+128 K_{18}^{r} n-16 K_{19}^{r}-16 K_{20}^{r} n-16 K_{23}^{r}-32 K_{28}^{r} \\
& -96 K_{3}^{r}-16 K_{33}^{r}+32 K_{37}^{r}-\frac{17}{36} L^{2} n^{2}-\frac{3 L^{2}}{8 n^{2}}-\frac{85 L^{2} n}{72}+\frac{3 L^{2}}{8 n}-\frac{43 L^{2}}{16}+24 L L_{0}^{r} n \\
& +\frac{8 L L_{0}^{r}}{n}+\frac{80 L L_{0}^{r}}{3}+\frac{176 L L_{1}^{r}}{3}+\frac{248 L L_{2}^{r}}{3}+\frac{4 L L_{3}^{r} n}{3}+\frac{8 L L_{3}^{r}}{n}+\frac{32 L L_{3}^{r}}{3}+\frac{32 L L_{4}^{r}}{3} \\
& +\frac{20 L L_{5}^{r} n}{3}+\frac{14 L L_{5}^{r}}{3}+48 L L_{6}^{r}+8 L L_{8}^{r} n+12 L L_{8}^{r}-64 L_{4}^{r} L_{5}^{r} n-32\left(L_{5}^{r}\right)^{2} \\
& +128 L_{5}^{r} L_{6}^{r} n+64 L_{5}^{r} L_{8}^{r} \\
& +\pi_{16}\left(-\frac{445 L n^{2}}{432}-\frac{317 L n}{108}-\frac{743 L}{216}+8 L_{0}^{r} n-\frac{8 L_{0}^{r}}{n}+\frac{272 L_{0}^{r}}{9}+\frac{80 L_{1}^{r}}{9}+\frac{512 L_{2}^{r}}{9}\right. \\
& \left.+\frac{16 L_{3}^{r} n}{9}-\frac{8 L_{3}^{r}}{n}+\frac{38 L_{3}^{r}}{9}+\frac{32 L_{4}^{r}}{9}+\frac{8 L_{5}^{r} n}{9}+\frac{26 L_{5}^{r}}{9}+48 L_{6}^{r}+8 L_{8}^{r} n+12 L_{8}^{r}\right) \\
& +\pi_{16}^{2}\left(-\frac{5}{72} n^{2} \pi^{2}-\frac{3865 n^{2}}{10368}+\frac{3}{4 n^{2}}-\frac{35 n \pi^{2}}{216}-\frac{1837 n}{3456}-\frac{3}{4 n}-\frac{91 \pi^{2}}{432}-\frac{853}{1296}\right) \\
& \gamma_{3}=2 K_{11}^{r}+8 K_{13}^{r}+16 K_{14}^{r} n-16 K_{17}^{r}-24 K_{18}^{r} n+16 K_{28}^{r}+48 K_{3}^{r}-4 K_{31}^{r}+8 K_{5}^{r} \\
& +2 K_{7}^{r}+4 K_{8}^{r} n+\frac{29 L^{2} n^{2}}{288}+\frac{23 L^{2} n}{72}+\frac{101 L^{2}}{96}-8 L L_{0}^{r} n+\frac{10 L L_{0}^{r}}{3 n}-16 L L_{0}^{r} \\
& -\frac{56 L L_{1}^{r}}{3}-40 L L_{2}^{r}-\frac{8 L L_{3}^{r} n}{3}+\frac{10 L L_{3}^{r}}{3 n}-6 L L_{3}^{r}-8 L L_{4}^{r}-L L_{5}^{r} n-2 L L_{5}^{r} \\
& +\pi_{16}\left(\frac{49 L n^{2}}{216}+\frac{503 L n}{864}+\frac{2867 L}{1728}-\frac{7 L_{0}^{r} n}{3}+\frac{28 L_{0}^{r}}{9 n}-\frac{43 L_{0}^{r}}{3}-\frac{62 L_{1}^{r}}{9}-31 L_{2}^{r}\right. \\
& \left.-\frac{25 L_{3}^{r} n}{18}+\frac{28 L_{3}^{r}}{9 n}-3 L_{3}^{r}-\frac{20 L_{4}^{r}}{3}-\frac{2 L_{5}^{r} n}{3}-\frac{5 L_{5}^{r}}{3}\right) \\
& +\pi_{16}^{2}\left(\frac{n^{2} \pi^{2}}{72}+\frac{445 n^{2}}{5184}+\frac{67 n \pi^{2}}{864}-\frac{59 n}{288}+\frac{185 \pi^{2}}{1728}+\frac{2705}{20736}\right) \\
& \gamma_{4}=2 K_{11}^{r}+8 K_{18}^{r} n+4 K_{31}^{r}+8 K_{5}^{r}+2 K_{7}^{r}+4 K_{8}^{r} n+\frac{11 L^{2} n^{2}}{288}-\frac{L^{2} n}{72}-\frac{7 L^{2}}{96}-\frac{4 L L_{0}^{r} n}{3} \\
& +\frac{10 L L_{0}^{r}}{3 n}-\frac{4 L L_{0}^{r}}{3}-\frac{4 L L_{2}^{r}}{3}-2 L L_{3}^{r} n+\frac{10 L L_{3}^{r}}{3 n}-\frac{4 L L_{3}^{r}}{3}+\frac{8 L L_{4}^{r}}{3}-\frac{L L_{5}^{r} n}{3}+\frac{2 L L_{5}^{r}}{3} \\
& +\pi_{16}\left(\frac{29 L n^{2}}{216}-\frac{23 L n}{864}-\frac{137 L}{1728}-\frac{13 L_{0}^{r} n}{9}+\frac{28 L_{0}^{r}}{9 n}-\frac{13 L_{0}^{r}}{9}-\frac{10 L_{1}^{r}}{3}-\frac{31 L_{2}^{r}}{9}\right. \\
& \left.-\frac{11 L_{3}^{r} n}{6}+\frac{28 L_{3}^{r}}{9 n}-\frac{19 L_{3}^{r}}{9}+\frac{20 L_{4}^{r}}{9}-\frac{4 L_{5}^{r} n}{9}+\frac{5 L_{5}^{r}}{9}\right) \\
& +\pi_{16}^{2}\left(-\frac{1}{108} n^{2} \pi^{2}+\frac{421 n^{2}}{3456}-\frac{n \pi^{2}}{288}-\frac{289 n}{10368}-\frac{11 \pi^{2}}{1728}-\frac{1091}{20736}\right) \\
& \gamma_{5}=K_{1}^{r}-8 K_{3}^{r}-4 K_{5}^{r}-\frac{5 L^{2} n^{2}}{1152}-\frac{55 L^{2} n}{2304}-\frac{5 L^{2}}{32}+\frac{5 L L_{0}^{r} n}{12}+\frac{5 L L_{0}^{r}}{2}+\frac{5 L L_{1}^{r}}{2} \\
& +\frac{25 L L_{2}^{r}}{4}+\frac{5 L L_{3}^{r} n}{24}+\frac{5 L L_{3}^{r}}{8} \\
& +\pi_{16}\left(-\frac{19 L n^{2}}{2304}-\frac{13 L n}{768}-\frac{307 L}{1152}+\frac{2 L_{0}^{r} n}{9}+\frac{7 L_{0}^{r}}{3}+\frac{7 L_{1}^{r}}{3}+\frac{35 L_{2}^{r}}{6}+\frac{5 L_{3}^{r} n}{18}+\frac{7 L_{3}^{r}}{12}\right)
\end{aligned}
$$




$$
\begin{aligned}
& +\pi_{16}^{2}\left(\frac{n^{2} \pi^{2}}{3456}-\frac{1015 n^{2}}{165888}-\frac{29 n \pi^{2}}{3456}+\frac{10313 n}{165888}-\frac{19 \pi^{2}}{1152}+\frac{71}{13824}\right) \\
\gamma_{6}= & 3 K_{1}^{r}-4 K_{5}^{r}-\frac{5}{384} L^{2} n^{2}+\frac{L^{2} n}{768}-\frac{5 L^{2}}{96}+\frac{7 L L_{0}^{r} n}{12}+\frac{5 L L_{0}^{r}}{6}+\frac{5 L L_{1}^{r}}{6} \\
& +\frac{25 L L_{2}^{r}}{12}+\frac{23 L L_{3}^{r} n}{24}+\frac{5 L L_{3}^{r}}{24} \\
& +\pi_{16}\left(-\frac{203 L n^{2}}{6912}+\frac{65 L n}{6912}-\frac{307 L}{3456}+\frac{4 L_{0}^{r} n}{9}+\frac{7 L_{0}^{r}}{9}+\frac{7 L_{1}^{r}}{9}+\frac{35 L_{2}^{r}}{18}+\frac{17 L_{3}^{r} n}{18}+\frac{7 L_{3}^{r}}{36}\right) \\
& +\pi_{16}^{2}\left(-\frac{n^{2} \pi^{2}}{3456}-\frac{1933 n^{2}}{165888}-\frac{11 n \pi^{2}}{3456}+\frac{5299 n}{165888}-\frac{19 \pi^{2}}{3456}+\frac{71}{41472}\right)
\end{aligned}
$$

The coefficients of polynomials part for $C_{P}$ at NNLO.

$$
\begin{aligned}
& \delta_{1}=128 K_{10}^{r} n-128 K_{18}^{r}+128 K_{2}^{r}-64 K_{20}^{r}-128 K_{21}^{r}-256 K_{22}^{r} n+128 K_{26}^{r}+384 K_{27}^{r} n \\
& -128 K_{35}^{r}+128 K_{40}^{r}+64 K_{9}^{r}-\frac{7 L^{2}}{4 n^{3}}+\frac{L^{2}}{n^{2}}+\frac{L^{2}}{2 n}+\frac{L^{2}}{2}-\frac{48 L L_{0}^{r}}{n^{2}}-64 L L_{1}^{r} n+\frac{64 L L_{1}^{r}}{n} \\
& -64 L L_{1}^{r}+\frac{16 L L_{2}^{r}}{n}-16 L L_{2}^{r}-\frac{48 L L_{3}^{r}}{n^{2}}+64 L L_{4}^{r} n-\frac{48 L L_{4}^{r}}{n}+48 L L_{4}^{r}+\frac{24 L L_{5}^{r}}{n^{2}} \\
& +8 L L_{5}^{r}-64 L L_{6}^{r} n+\frac{32 L L_{6}^{r}}{n}-32 L L_{6}^{r}+\frac{32 L L_{7}^{r}}{n}-\frac{48 L L_{8}^{r}}{n^{2}}-16 L L_{8}^{r}-512\left(L_{4}^{r}\right)^{2} n \\
& -256 L_{4}^{r} L_{5}^{r}+2048 L_{4}^{r} L_{6}^{r} n+512 L_{4}^{r} L_{8}^{r}+512 L_{5}^{r} L_{6}^{r}-2048\left(L_{6}^{r}\right)^{2} n-1024 L_{6}^{r} L_{8}^{r} \\
& +\pi_{16}\left(-\frac{3 L}{2 n^{3}}+\frac{L}{n^{2}}+\frac{L}{n}-L-\frac{16 L_{0}^{r}}{n^{2}}+\frac{32 L_{1}^{r}}{n}-32 L_{1}^{r}-\frac{16 L_{3}^{r}}{n^{2}}-\frac{32 L_{4}^{r}}{n}+32 L_{4}^{r}\right. \\
& \left.+\frac{16 L_{5}^{r}}{n^{2}}+\frac{32 L_{6}^{r}}{n}-32 L_{6}^{r}-\frac{48 L_{8}^{r}}{n^{2}}\right) \\
& +\pi_{16}^{2}\left(\frac{5}{4 n^{3}}-\frac{1}{2 n^{2}}+\frac{3}{4 n}-\frac{1}{6}\right) \\
& \delta_{2}=-128 K_{10}^{r} n+128 K_{18}^{r}-192 K_{2}^{r}+32 K_{20}^{r}+64 K_{21}^{r}+128 K_{22}^{r} n-32 K_{32}^{r}+64 K_{35}^{r} \\
& -32 K_{38}^{r}-64 K_{9}^{r}+\frac{37 L^{2} n}{72}-\frac{3 L^{2}}{4 n}-\frac{7 L^{2}}{36}+\frac{104 L L_{0}^{r}}{3 n^{2}}-8 L L_{0}^{r}-\frac{48 L L_{1}^{r}}{n}+48 L L_{1}^{r} \\
& -16 L L_{2}^{r} n-\frac{32 L L_{2}^{r}}{3 n}+\frac{32 L L_{2}^{r}}{3}+\frac{104 L L_{3}^{r}}{3 n^{2}}-\frac{64 L L_{3}^{r}}{3}+16 L L_{4}^{r} n+\frac{16 L L_{4}^{r}}{n}-16 L L_{4}^{r} \\
& +12 L L_{5}^{r}-32 L L_{6}^{r} n-24 L L_{8}^{r}+256\left(L_{4}^{r}\right)^{2} n+128 L_{4}^{r} L_{5}^{r}-512 L_{4}^{r} L_{6}^{r} n-256 L_{4}^{r} L_{8}^{r} \\
& +\pi_{16}\left(-\frac{31 L n}{24}-\frac{L}{4 n}-\frac{5 L}{18}+\frac{152 L_{0}^{r}}{9 n^{2}}-32 L_{1}^{r} n-\frac{32 L_{1}^{r}}{n}+32 L_{1}^{r}-\frac{8 L_{2}^{r}}{9 n}+\frac{8 L_{2}^{r}}{9}\right. \\
& \left.+\frac{152 L_{3}^{r}}{9 n^{2}}-\frac{88 L_{3}^{r}}{9}+32 L_{4}^{r} n+\frac{16 L_{4}^{r}}{n}-16 L_{4}^{r}+12 L_{5}^{r}-32 L_{6}^{r} n-24 L_{8}^{r}\right) \\
& +\pi_{16}^{2}\left(-\frac{n \pi^{2}}{27}-\frac{\pi^{2}}{12 n}-\frac{373 n}{2592}+\frac{25}{16 n}-\frac{5 \pi^{2}}{216}-\frac{565}{648}\right) \\
& \delta_{3}=32 K_{10}^{r} n+4 K_{15}^{r}+8 K_{16}^{r} n-32 K_{18}^{r}+96 K_{2}^{r}-8 K_{29}^{r}+16 K_{32}^{r}+16 K_{9}^{r}-\frac{13 L^{2} n}{72}
\end{aligned}
$$$$
+\frac{49 L^{2}}{144}-\frac{20 L L_{0}^{r}}{3 n^{2}}+\frac{10 L L_{0}^{r}}{3}+32 L L_{1}^{r} n+\frac{8 L L_{1}^{r}}{n}-8 L L_{1}^{r}+\frac{20 L L_{2}^{r} n}{3}+\frac{8 L L_{2}^{r}}{3 n}
$$$$
-\frac{8 L L_{2}^{r}}{3}-\frac{20 L L_{3}^{r}}{3 n^{2}}+\frac{44 L L_{3}^{r}}{3}-16 L L_{4}^{r} n-3 L L_{5}^{r}
$$ 


$$
\begin{aligned}
& +\pi_{16}\left(\frac{11 L n}{24}+\frac{115 L}{144}-\frac{56 L_{0}^{r}}{9 n^{2}}+\frac{4 L_{0}^{r}}{9}+32 L_{1}^{r} n+\frac{8 L_{1}^{r}}{n}-8 L_{1}^{r}+\frac{8 L_{2}^{r} n}{9}+\frac{20 L_{2}^{r}}{9 n}\right. \\
& \left.-\frac{20 L_{2}^{r}}{9}-\frac{56 L_{3}^{r}}{9 n^{2}}+\frac{83 L_{3}^{r}}{9}-16 L_{4}^{r} n-3 L_{5}^{r}\right) \\
& +\pi_{16}^{2}\left(-\frac{25 n \pi^{2}}{864}+\frac{625 n}{2592}+\frac{17 \pi^{2}}{864}+\frac{1451}{5184}\right) \\
\delta_{4}= & 4 K_{15}^{r}+8 K_{16}^{r} n+8 K_{29}^{r}+\frac{5 L^{2} n}{48}+\frac{L^{2}}{24}-2 L L_{0}^{r}-4 L L_{2}^{r} n-L L_{5}^{r} \\
& +\pi_{16}\left(\frac{L n}{16}+\frac{7 L}{48}+L_{3}^{r}-L_{5}^{r}\right) \\
& +\pi_{16}^{2}\left(\frac{n \pi^{2}}{288}+\frac{7 n}{64}+\frac{\pi^{2}}{288}+\frac{11}{192}\right) \\
\delta_{5}= & -16 K_{2}^{r}+2 K_{4}^{r}+2 K_{6}^{r}+\frac{55 L^{2} n}{384}-\frac{L^{2}}{48}-\frac{11 L L_{0}^{r}}{6}-8 L L_{1}^{r} n-\frac{8 L L_{2}^{r} n}{3}-\frac{17 L L_{3}^{r}}{6} \\
& +\pi_{16}\left(\frac{101 L n}{384}-\frac{13 L}{384}-\frac{29 L_{0}^{r}}{18}-8 L_{1}^{r} n-\frac{20 L_{2}^{r} n}{9}-\frac{97 L_{3}^{r}}{36}\right) \\
& +\pi_{16}^{2}\left(\frac{19 n \pi^{2}}{1152}-\frac{115 n}{13824}+\frac{\pi^{2}}{1152}-\frac{349}{13824}\right) \\
& +\pi_{16}^{2}\left(\frac{5 n \pi^{2}}{1152}-\frac{437 n}{13824}-\frac{\pi^{2}}{1152}+\frac{349}{13824}\right) \\
\delta_{6}= & +2 K_{6}^{r}+\frac{5 L^{2} n}{384}+\frac{L^{2}}{48}-\frac{3 L L_{0}^{r}}{2}-\frac{L L_{3}^{r}}{2} \\
& +\pi_{16}\left(\frac{L n}{128}+\frac{13 L}{384}-\frac{3 L_{0}^{r}}{2}-\frac{5 L_{3}^{r}}{12}\right) \\
&
\end{aligned}
$$

\section{B.3 Pseudo-real or two-colour}

The coefficients of polynomials part for $B_{P}$ at NNLO.

$$
\begin{aligned}
\gamma_{1}= & 32 K_{13}^{r}+64 K_{14}^{r} n-96 K_{17}^{r}-192 K_{18}^{r} n+96 K_{25}^{r}+64 K_{26}^{r} n+64 K_{3}^{r}-64 K_{37}^{r} \\
& +96 K_{39}^{r}+64 K_{40}^{r} n+\frac{29 L^{2} n^{2}}{36}-\frac{83 L^{2} n}{36}+\frac{19 L^{2}}{4 n^{2}}+\frac{17 L^{2}}{4 n}+\frac{19 L^{2}}{12}-\frac{80 L L_{0}^{r} n}{3} \\
& +\frac{32 L L_{0}^{r}}{3 n}+\frac{80 L L_{0}^{r}}{3}-64 L L_{1}^{r}-\frac{224 L L_{2}^{r}}{3}-8 L L_{3}^{r} n+\frac{32 L L_{3}^{r}}{3 n}+\frac{56 L L_{3}^{r}}{3}+\frac{64 L L_{4}^{r}}{3} \\
& +\frac{40 L L_{5}^{r} n}{3}-\frac{48 L L_{5}^{r}}{n}-\frac{64 L L_{5}^{r}}{3}-160 L L_{6}^{r}-32 L L_{7}^{r}-64 L L_{8}^{r} n+\frac{112 L L_{8}^{r}}{n} \\
& +80 L L_{8}^{r}+512 L_{4}^{r} L_{8}^{r} n+256 L_{5}^{r} L_{8}^{r}-1024 L_{6}^{r} L_{8}^{r} n-512\left(L_{8}^{r}\right)^{2} \\
& +\pi_{16}\left(\frac{229 L n^{2}}{216}-\frac{623 L n}{216}+\frac{L}{n^{2}}+\frac{L}{n}+\frac{155 L}{108}-\frac{80 L_{0}^{r} n}{9}+\frac{128 L_{0}^{r}}{9 n}+\frac{224 L_{0}^{r}}{9}\right. \\
& -\frac{32 L_{1}^{r}}{3}-\frac{368 L_{2}^{r}}{9}-\frac{8 L_{3}^{r} n}{3}+\frac{128 L_{3}^{r}}{9 n}+\frac{56 L_{3}^{r}}{9}+\frac{256 L_{4}^{r}}{9}+\frac{64 L_{5}^{r} n}{9} \\
& \left.-\frac{32 L_{5}^{r}}{n}-\frac{136 L_{5}^{r}}{9}-128 L_{6}^{r}-32 L_{8}^{r} n+\frac{96 L_{8}^{r}}{n}+64 L_{8}^{r}\right)
\end{aligned}
$$




$$
\begin{aligned}
& +\pi_{16}^{2}\left(\frac{n^{2} \pi^{2}}{27}+\frac{1645 n^{2}}{1728}-\frac{10763 n}{5184}-\frac{35}{8 n^{2}}-\frac{27}{8 n}+\frac{13 \pi^{2}}{54}-\frac{2149}{1296}\right) \\
& \gamma_{2}=-32 K_{13}^{r}-64 K_{14}^{r} n+64 K_{17}^{r}+128 K_{18}^{r} n-16 K_{19}^{r}-16 K_{20}^{r} n-16 K_{23}^{r}-32 K_{28}^{r} \\
& -96 K_{3}^{r}-16 K_{33}^{r}+32 K_{37}^{r}-\frac{17}{36} L^{2} n^{2}+\frac{85 L^{2} n}{72}-\frac{3 L^{2}}{8 n^{2}}-\frac{3 L^{2}}{8 n}-\frac{43 L^{2}}{16}+24 L L_{0}^{r} n \\
& +\frac{8 L L_{0}^{r}}{n}-\frac{80 L L_{0}^{r}}{3}+\frac{176 L L_{1}^{r}}{3}+\frac{248 L L_{2}^{r}}{3}+\frac{4 L L_{3}^{r} n}{3}+\frac{8 L L_{3}^{r}}{n}-\frac{32 L L_{3}^{r}}{3}+\frac{32 L L_{4}^{r}}{3} \\
& +\frac{20 L L_{5}^{r} n}{3}-\frac{14 L L_{5}^{r}}{3}+48 L L_{6}^{r}+8 L L_{8}^{r} n-12 L L_{8}^{r}-64 L_{4}^{r} L_{5}^{r} n \\
& -32\left(L_{5}^{r}\right)^{2}+128 L_{5}^{r} L_{6}^{r} n+64 L_{5}^{r} L_{8}^{r} \\
& +\pi_{16}\left(-\frac{445 L n^{2}}{432}+\frac{317 L n}{108}-\frac{743 L}{216}+8 L_{0}^{r} n-\frac{8 L_{0}^{r}}{n}-\frac{272 L_{0}^{r}}{9}+\frac{80 L_{1}^{r}}{9}\right. \\
& \left.+\frac{512 L_{2}^{r}}{9}+\frac{16 L_{3}^{r} n}{9}-\frac{8 L_{3}^{r}}{n}-\frac{38 L_{3}^{r}}{9}+\frac{32 L_{4}^{r}}{9}+\frac{8 L_{5}^{r} n}{9}-\frac{26 L_{5}^{r}}{9}+48 L_{6}^{r}+8 L_{8}^{r} n-12 L_{8}^{r}\right) \\
& +\pi_{16}^{2}\left(-\frac{5}{72} n^{2} \pi^{2}-\frac{3865 n^{2}}{10368}+\frac{35 n \pi^{2}}{216}+\frac{1837 n}{3456}+\frac{3}{4 n^{2}}+\frac{3}{4 n}-\frac{91 \pi^{2}}{432}-\frac{853}{1296}\right) \\
& \gamma_{3}=2 K_{11}^{r}+8 K_{13}^{r}+16 K_{14}^{r} n-16 K_{17}^{r}-24 K_{18}^{r} n+16 K_{28}^{r}+48 K_{3}^{r}-4 K_{31}^{r}+8 K_{5}^{r} \\
& +2 K_{7}^{r}+4 K_{8}^{r} n+\frac{29 L^{2} n^{2}}{288}-\frac{23 L^{2} n}{72}+\frac{101 L^{2}}{96}-8 L L_{0}^{r} n+\frac{10 L L_{0}^{r}}{3 n}+16 L L_{0}^{r} \\
& -\frac{56 L L_{1}^{r}}{3}-40 L L_{2}^{r}-\frac{8 L L_{3}^{r} n}{3}+\frac{10 L L_{3}^{r}}{3 n}+6 L L_{3}^{r}-8 L L_{4}^{r}-L L_{5}^{r} n+2 L L_{5}^{r} \\
& +\pi_{16}\left(\frac{49 L n^{2}}{216}-\frac{503 L n}{864}+\frac{2867 L}{1728}-\frac{7 L_{0}^{r} n}{3}+\frac{28 L_{0}^{r}}{9 n}+\frac{43 L_{0}^{r}}{3}-\frac{62 L_{1}^{r}}{9}-31 L_{2}^{r}\right. \\
& \left.-\frac{25 L_{3}^{r} n}{18}+\frac{28 L_{3}^{r}}{9 n}+3 L_{3}^{r}-\frac{20 L_{4}^{r}}{3}-\frac{2 L_{5}^{r} n}{3}+\frac{5 L_{5}^{r}}{3}\right) \\
& +\pi_{16}^{2}\left(\frac{n^{2} \pi^{2}}{72}+\frac{445 n^{2}}{5184}-\frac{67 n \pi^{2}}{864}+\frac{59 n}{288}+\frac{185 \pi^{2}}{1728}+\frac{2705}{20736}\right) \\
& \gamma_{4}=2 K_{11}^{r}+8 K_{18}^{r} n+4 K_{31}^{r}+8 K_{5}^{r}+2 K_{7}^{r}+4 K_{8}^{r} n+\frac{11 L^{2} n^{2}}{288}+\frac{L^{2} n}{72}-\frac{7 L^{2}}{96}-\frac{4 L L_{0}^{r} n}{3} \\
& +\frac{10 L L_{0}^{r}}{3 n}+\frac{4 L L_{0}^{r}}{3}-\frac{4 L L_{2}^{r}}{3}-2 L L_{3}^{r} n+\frac{10 L L_{3}^{r}}{3 n}+\frac{4 L L_{3}^{r}}{3}+\frac{8 L L_{4}^{r}}{3}-\frac{L L_{5}^{r} n}{3}-\frac{2 L L_{5}^{r}}{3} \\
& +\pi_{16}\left(\frac{29 L n^{2}}{216}+\frac{23 L n}{864}-\frac{137 L}{1728}-\frac{13 L_{0}^{r} n}{9}+\frac{28 L_{0}^{r}}{9 n}+\frac{13 L_{0}^{r}}{9}-\frac{10 L_{1}^{r}}{3}-\frac{31 L_{2}^{r}}{9}\right. \\
& \left.-\frac{11 L_{3}^{r} n}{6}+\frac{28 L_{3}^{r}}{9 n}+\frac{19 L_{3}^{r}}{9}+\frac{20 L_{4}^{r}}{9}-\frac{4 L_{5}^{r} n}{9}-\frac{5 L_{5}^{r}}{9}\right) \\
& +\pi_{16}^{2}\left(-\frac{1}{108} n^{2} \pi^{2}+\frac{421 n^{2}}{3456}+\frac{n \pi^{2}}{288}+\frac{289 n}{10368}-\frac{11 \pi^{2}}{1728}-\frac{1091}{20736}\right) \\
& \gamma_{5}=K_{1}^{r}-8 K_{3}^{r}-4 K_{5}^{r}-\frac{5 L^{2} n^{2}}{1152}+\frac{55 L^{2} n}{2304}-\frac{5 L^{2}}{32}+\frac{5 L L_{0}^{r} n}{12}-\frac{5 L L_{0}^{r}}{2} \\
& +\frac{5 L L_{1}^{r}}{2}+\frac{25 L L_{2}^{r}}{4}+\frac{5 L L_{3}^{r} n}{24}-\frac{5 L L_{3}^{r}}{8} \\
& +\pi_{16}\left(-\frac{19 L n^{2}}{2304}+\frac{13 L n}{768}-\frac{307 L}{1152}+\frac{2 L_{0}^{r} n}{9}-\frac{7 L_{0}^{r}}{3}+\frac{7 L_{1}^{r}}{3}+\frac{35 L_{2}^{r}}{6}+\frac{5 L_{3}^{r} n}{18}-\frac{7 L_{3}^{r}}{12}\right)
\end{aligned}
$$




$$
\begin{aligned}
& +\pi_{16}^{2}\left(\frac{n^{2} \pi^{2}}{3456}-\frac{1015 n^{2}}{165888}+\frac{29 n \pi^{2}}{3456}-\frac{10313 n}{165888}-\frac{19 \pi^{2}}{1152}+\frac{71}{13824}\right) \\
\gamma_{6}= & 3 K_{1}^{r}-4 K_{5}^{r}-\frac{5}{384} L^{2} n^{2}-\frac{L^{2} n}{768}-\frac{5 L^{2}}{96}+\frac{7 L L_{0}^{r} n}{12}-\frac{5 L L_{0}^{r}}{6}+\frac{5 L L_{1}^{r}}{6} \\
& +\frac{25 L L_{2}^{r}}{12}+\frac{23 L L_{3}^{r} n}{24}-\frac{5 L L_{3}^{r}}{24} \\
& +\pi_{16}\left(-\frac{203 L n^{2}}{6912}-\frac{65 L n}{6912}-\frac{307 L}{3456}+\frac{4 L_{0}^{r} n}{9}-\frac{7 L_{0}^{r}}{9}+\frac{7 L_{1}^{r}}{9}+\frac{35 L_{2}^{r}}{18}+\frac{17 L_{3}^{r} n}{18}-\frac{7 L_{3}^{r}}{36}\right) \\
& +\pi_{16}^{2}\left(-\frac{n^{2} \pi^{2}}{3456}-\frac{1933 n^{2}}{165888}+\frac{11 n \pi^{2}}{3456}-\frac{5299 n}{165888}-\frac{19 \pi^{2}}{3456}+\frac{71}{41472}\right)
\end{aligned}
$$

The coefficients of polynomials part for $C_{P}$ at NNLO.

$$
\begin{aligned}
& \delta_{1}=128 K_{10}^{r} n-128 K_{18}^{r}+128 K_{2}^{r}-64 K_{20}^{r}-128 K_{21}^{r}-256 K_{22}^{r} n+128 K_{26}^{r} \\
& +384 K_{27}^{r} n-128 K_{35}^{r}+128 K_{40}^{r}+64 K_{9}^{r}-\frac{7 L^{2}}{4 n^{3}}-\frac{L^{2}}{n^{2}}+\frac{L^{2}}{2 n}-\frac{L^{2}}{2}-\frac{48 L L_{0}^{r}}{n^{2}} \\
& -64 L L_{1}^{r} n+\frac{64 L L_{1}^{r}}{n}+64 L L_{1}^{r}+\frac{16 L L_{2}^{r}}{n}+16 L L_{2}^{r}-\frac{48 L L_{3}^{r}}{n^{2}}+64 L L_{4}^{r} n \\
& -\frac{48 L L_{4}^{r}}{n}-48 L L_{4}^{r}+\frac{24 L L_{5}^{r}}{n^{2}}+8 L L_{5}^{r}-64 L L_{6}^{r} n+\frac{32 L L_{6}^{r}}{n}+32 L L_{6}^{r}+\frac{32 L L_{7}^{r}}{n} \\
& -\frac{48 L L_{8}^{r}}{n^{2}}-16 L L_{8}^{r}-512\left(L_{4}^{r}\right)^{2} n-256 L_{4}^{r} L_{5}^{r}+2048 L_{4}^{r} L_{6}^{r} n+512 L_{4}^{r} L_{8}^{r} \\
& +512 L_{5}^{r} L_{6}^{r}-2048\left(L_{6}^{r}\right)^{2} n-1024 L_{6}^{r} L_{8}^{r} \\
& +\pi_{16}\left(-\frac{3 L}{2 n^{3}}-\frac{L}{n^{2}}+\frac{L}{n}+L-\frac{16 L_{0}^{r}}{n^{2}}+\frac{32 L_{1}^{r}}{n}+32 L_{1}^{r}-\frac{16 L_{3}^{r}}{n^{2}}-\frac{32 L_{4}^{r}}{n}\right. \\
& \left.-32 L_{4}^{r}+\frac{16 L_{5}^{r}}{n^{2}}+\frac{32 L_{6}^{r}}{n}+32 L_{6}^{r}-\frac{48 L_{8}^{r}}{n^{2}}\right) \\
& +\left(\frac{5}{4 n^{3}}+\frac{1}{2 n^{2}}+\frac{3}{4 n}+\frac{1}{6}\right) \pi_{16}^{2} \\
& \delta_{2}=-128 K_{10}^{r} n+128 K_{18}^{r}-192 K_{2}^{r}+32 K_{20}^{r}+64 K_{21}^{r}+128 K_{22}^{r} n-32 K_{32}^{r}+64 K_{35}^{r} \\
& -32 K_{38}^{r}-64 K_{9}^{r}+\frac{37 L^{2} n}{72}-\frac{3 L^{2}}{4 n}+\frac{7 L^{2}}{36}+\frac{104 L L_{0}^{r}}{3 n^{2}}-8 L L_{0}^{r}-\frac{48 L L_{1}^{r}}{n}-48 L L_{1}^{r} \\
& -16 L L_{2}^{r} n-\frac{32 L L_{2}^{r}}{3 n}-\frac{32 L L_{2}^{r}}{3}+\frac{104 L L_{3}^{r}}{3 n^{2}}-\frac{64 L L_{3}^{r}}{3}+16 L L_{4}^{r} n+\frac{16 L L_{4}^{r}}{n}+16 L L_{4}^{r} \\
& +12 L L_{5}^{r}-32 L L_{6}^{r} n-24 L L_{8}^{r}+256\left(L_{4}^{r}\right)^{2} n+128 L_{4}^{r} L_{5}^{r}-512 L_{4}^{r} L_{6}^{r} n-256 L_{4}^{r} L_{8}^{r} \\
& +\pi_{16}\left(-\frac{31 L n}{24}-\frac{L}{4 n}+\frac{5 L}{18}+\frac{152 L_{0}^{r}}{9 n^{2}}-32 L_{1}^{r} n-\frac{32 L_{1}^{r}}{n}-32 L_{1}^{r}-\frac{8 L_{2}^{r}}{9 n}\right. \\
& \left.-\frac{8 L_{2}^{r}}{9}+\frac{152 L_{3}^{r}}{9 n^{2}}-\frac{88 L_{3}^{r}}{9}+32 L_{4}^{r} n+\frac{16 L_{4}^{r}}{n}+16 L_{4}^{r}+12 L_{5}^{r}-32 L_{6}^{r} n-24 L_{8}^{r}\right) \\
& +\pi_{16}^{2}\left(-\frac{n \pi^{2}}{27}-\frac{373 n}{2592}-\frac{\pi^{2}}{12 n}+\frac{25}{16 n}+\frac{5 \pi^{2}}{216}+\frac{565}{648}\right) \\
& \delta_{3}=32 K_{10}^{r} n+4 K_{15}^{r}+8 K_{16}^{r} n-32 K_{18}^{r}+96 K_{2}^{r}-8 K_{29}^{r}+16 K_{32}^{r}+16 K_{9}^{r}-\frac{13 L^{2} n}{72} \\
& -\frac{49 L^{2}}{144}-\frac{20 L L_{0}^{r}}{3 n^{2}}+\frac{10 L L_{0}^{r}}{3}+32 L L_{1}^{r} n+\frac{8 L L_{1}^{r}}{n}+8 L L_{1}^{r}+\frac{20 L L_{2}^{r} n}{3}
\end{aligned}
$$




$$
\begin{aligned}
& +\frac{8 L L_{2}^{r}}{3 n}+\frac{8 L L_{2}^{r}}{3}-\frac{20 L L_{3}^{r}}{3 n^{2}}+\frac{44 L L_{3}^{r}}{3}-16 L L_{4}^{r} n-3 L L_{5}^{r} \\
& +\pi_{16}\left(\frac{11 L n}{24}-\frac{115 L}{144}-\frac{56 L_{0}^{r}}{9 n^{2}}+\frac{4 L_{0}^{r}}{9}+32 L_{1}^{r} n+\frac{8 L_{1}^{r}}{n}+8 L_{1}^{r}+\frac{8 L_{2}^{r} n}{9}+\frac{20 L_{2}^{r}}{9 n}\right. \\
& \left.+\frac{20 L_{2}^{r}}{9}-\frac{56 L_{3}^{r}}{9 n^{2}}+\frac{83 L_{3}^{r}}{9}-16 L_{4}^{r} n-3 L_{5}^{r}\right) \\
& +\pi_{16}^{2}\left(-\frac{25 n \pi^{2}}{864}+\frac{625 n}{2592}-\frac{17 \pi^{2}}{864}-\frac{1451}{5184}\right) \\
\delta_{4}= & 4 K_{15}^{r}+8 K_{16}^{r} n+8 K_{29}^{r}+\frac{5 L^{2} n}{48}-\frac{L^{2}}{24}-2 L L_{0}^{r}-4 L L_{2}^{r} n-L L_{5}^{r} \\
& +\pi_{16}\left(\frac{L n}{16}-\frac{7 L}{48}+L_{3}^{r}-L_{5}^{r}\right)+\pi_{16}^{2}\left(\frac{n \pi^{2}}{288}+\frac{7 n}{64}-\frac{\pi^{2}}{288}-\frac{11}{192}\right) \\
\delta_{5}= & -16 K_{2}^{r}+2 K_{4}^{r}+2 K_{6}^{r}+\frac{55 L^{2} n}{384}+\frac{L^{2}}{48}-\frac{11 L L_{0}^{r}}{6}-8 L L_{1}^{r} n-\frac{8 L L_{2}^{r} n}{3}-\frac{17 L L_{3}^{r}}{6} \\
& +\pi_{16}\left(\frac{101 L n}{384}+\frac{13 L}{384}-\frac{29 L_{0}^{r}}{18}-8 L_{1}^{r} n-\frac{20 L_{2}^{r} n}{9}-\frac{97 L_{3}^{r}}{36}\right) \\
& +\pi_{16}^{2}\left(\frac{19 n \pi^{2}}{1152}-\frac{115 n}{13824}-\frac{\pi^{2}}{1152}+\frac{349}{13824}\right) \\
& +\pi_{16}^{2}\left(\frac{5 n \pi^{2}}{1152}-\frac{437 n}{13824}+\frac{\pi^{2}}{1152}-\frac{349}{13824}\right) \\
\delta_{6}= & 2 K_{6}^{r}+\frac{5 L^{2} n}{384}-\frac{L^{2}}{48}-\frac{3 L L_{0}^{r}}{2}-\frac{L L_{3}^{r}}{2} \\
& +\pi_{16}\left(\frac{L n}{128}-\frac{13 L}{384}-\frac{3 L_{0}^{r}}{2}-\frac{5 L_{3}^{r}}{12}\right) \\
&
\end{aligned}
$$

\section{Scattering lengths}

\section{C.1 Complex or QCD case}

$$
\begin{aligned}
\pi a_{0}^{I}= & x_{2}\left(-\frac{1}{16 n}+\frac{n}{8}\right) \\
+ & x_{2}^{2}\left(-\frac{2}{n} \alpha_{4}-\frac{1}{n} \alpha_{3}-\frac{1}{4 n} \alpha_{2}-\frac{3}{16 n} \alpha_{1}+\beta_{4}-\frac{1}{2} \beta_{3}-\frac{1}{8} \beta_{2}+\frac{1}{32} \beta_{1}\right. \\
& \left.+2 n \alpha_{4}+\frac{n}{8} \alpha_{1}+\frac{n^{2}}{2} \beta_{3}+\frac{n^{2}}{8} \beta_{2}+\frac{n^{2}}{32} \beta_{1}\right) \\
+ & \pi_{16} x_{2}^{2}\left(-\frac{1}{2}+\frac{1}{8 n^{2}}+\frac{n^{2}}{2}\right) \\
+ & x_{2}^{3}\left(-\frac{4}{n} \gamma_{5}-\frac{2}{n} \gamma_{4}-\frac{1}{n} \gamma_{3}-\frac{1}{4 n} \gamma_{2}-\frac{3}{16 n} \gamma_{1}-2 \delta_{5}+\delta_{4}-\frac{1}{2} \delta_{3}-\frac{1}{8} \delta_{2}\right. \\
& \left.\quad+\frac{1}{32} \delta_{1}+2 n \gamma_{4}+\frac{n}{8} \gamma_{1}+2 n^{2} \delta_{5}+\frac{n^{2}}{2} \delta_{3}+\frac{n^{2}}{8} \delta_{2}+\frac{n^{2}}{32} \delta_{1}\right)
\end{aligned}
$$




$$
\begin{aligned}
& +\pi_{16} x_{2}^{3}\left(\frac{7}{4 n^{3}} L+\frac{12}{n^{2}} L_{8}^{r}-\frac{4}{n^{2}} L_{5}^{r}+\frac{12}{n^{2}} L_{3}^{r}+\frac{12}{n^{2}} L_{0}^{r}-\frac{5}{n} L-\frac{4}{n} L_{6}^{r}\right. \\
& +\frac{12}{n} L_{4}^{r}-\frac{4}{n} L_{2}^{r}-\frac{4}{n} L_{1}^{r}-32 L_{8}^{r}+8 L_{5}^{r}-32 L_{3}^{r}-32 L_{0}^{r}+\frac{17 n}{4} L \\
& +4 n L_{6}^{r}-28 n L_{4}^{r}+4 n L_{2}^{r}+4 n L_{1}^{r}+16 n^{2} L_{8}^{r}+16 n^{2} L_{3}^{r} \\
& \left.+16 n^{2} L_{0}^{r}-\frac{5 n^{3}}{2} L+8 n^{3} L_{6}^{r}+8 n^{3} L_{4}^{r}+8 n^{3} L_{2}^{r}+8 n^{3} L_{1}^{r}\right) \\
& +\pi_{16}^{2} x_{2}^{3}\left[-\frac{3}{n^{3}}+\frac{7}{n}-\frac{49 n}{24}+\frac{n^{3}}{12}+\pi^{2}\left(\frac{1}{2 n^{3}}-\frac{7}{6 n}+\frac{53 n}{144}-\frac{5 n^{3}}{72}\right)\right], \\
& \pi a_{0}^{S}=+x_{2}\left(-\frac{1}{8 n}+\frac{n}{16}\right) \\
& +x_{2}^{2}\left(-\frac{4}{n} \alpha_{4}-\frac{2}{n} \alpha_{3}-\frac{1}{2 n} \alpha_{2}-\frac{3}{8 n} \alpha_{1}+\beta_{4}+\frac{1}{16} \beta_{1}+n \alpha_{4}+\frac{n}{16} \alpha_{1}\right) \\
& +\pi_{16} x_{2}^{2}\left(-\frac{1}{2}+\frac{1}{2 n^{2}}+\frac{n^{2}}{8}\right) \\
& +x_{2}^{3}\left(-\frac{8}{n} \gamma_{5}-\frac{4}{n} \gamma_{4}-\frac{2}{n} \gamma_{3}-\frac{1}{2 n} \gamma_{2}-\frac{3}{8 n} \gamma_{1}+\delta_{4}+\frac{1}{16} \delta_{1}+n \gamma_{4}+\frac{n}{16} \gamma_{1}\right) \\
& +\pi_{16} x_{2}^{3}\left(\frac{7}{n^{3}} L+\frac{48}{n^{2}} L_{8}^{r}-\frac{16}{n^{2}} L_{5}^{r}+\frac{48}{n^{2}} L_{3}^{r}+\frac{48}{n^{2}} L_{0}^{r}-\frac{11}{2 n} L-\frac{16}{n} L_{6}^{r}\right. \\
& +\frac{16}{n} L_{4}^{r}-\frac{16}{n} L_{2}^{r}-\frac{16}{n} L_{1}^{r}-32 L_{8}^{r}+8 L_{5}^{r}-32 L_{3}^{r}-32 L_{0}^{r}+\frac{3 n}{2} L \\
& +8 n L_{6}^{r}-8 n L_{4}^{r}+8 n L_{2}^{r}+8 n L_{1}^{r}+4 n^{2} L_{8}^{r}+4 n^{2} L_{3}^{r}+4 n^{2} L_{0}^{r} \\
& \left.-\frac{n^{3}}{4} L\right) \\
& +\pi_{16}^{2} x_{2}^{3}\left[-\frac{10}{n^{3}}+\frac{9}{2 n}+\frac{5 n}{6}-\frac{7 n^{3}}{24}+\pi^{2}\left(\frac{5}{3 n^{3}}-\frac{3}{4 n}-\frac{n}{9}+\frac{5 n^{3}}{144}\right)\right], \\
& \pi a_{1}^{A}=+x_{2}\left(\frac{n}{48}\right) \\
& +x_{2}^{2}\left(\frac{1}{3} \beta_{4}+\frac{1}{24} \beta_{2}-\frac{n}{3} \alpha_{4}-\frac{n}{24} \alpha_{2}\right) \\
& +\pi_{16} x_{2}^{2}\left(-\frac{1}{72}+\frac{1}{72 n^{2}}-\frac{n^{2}}{432}\right) \\
& +x_{2}^{3}\left(\frac{2}{3} \delta_{6}+\frac{1}{3} \delta_{4}+\frac{1}{24} \delta_{2}-\frac{2 n}{3} \gamma_{6}-\frac{n}{3} \gamma_{4}-\frac{n}{24} \gamma_{2}\right) \\
& +\pi_{16} x_{2}^{3}\left(\frac{7}{36 n^{3}} L+\frac{4}{3 n^{2}} L_{8}^{r}-\frac{4}{9 n^{2}} L_{5}^{r}+\frac{20}{27 n^{2}} L_{3}^{r}+\frac{20}{27 n^{2}} L_{0}^{r}-\frac{1}{9 n} L\right. \\
& -\frac{4}{9 n} L_{6}^{r}+\frac{4}{9 n} L_{4}^{r}-\frac{4}{27 n} L_{2}^{r}-\frac{4}{9 n} L_{1}^{r}-\frac{8}{9} L_{8}^{r}+\frac{2}{9} L_{5}^{r}-\frac{4}{9} L_{3}^{r}-\frac{16}{27} L_{0}^{r} \\
& \left.+\frac{49 n}{648} L-\frac{4 n}{9} L_{6}^{r}-\frac{4 n}{27} L_{4}^{r}-\frac{8 n}{27} L_{2}^{r}-\frac{4 n}{27} L_{1}^{r}-\frac{n^{2}}{27} L_{5}^{r}+\frac{n^{3}}{432} L\right)
\end{aligned}
$$




$$
\begin{aligned}
& +\pi_{16}^{2} x_{2}^{3}\left[\frac{1}{12 n^{3}}+\frac{1}{8 n}-\frac{7 n}{54}-\frac{25 n^{3}}{5184}+\pi^{2}\left(\frac{1}{54 n^{3}}-\frac{5}{216 n}+\frac{25 n}{1296}-\frac{n^{3}}{1296}\right)\right] \\
& \pi a_{1}^{S A}=+x_{2}^{2}\left(\frac{1}{3} \beta_{4}+\frac{1}{24} \beta_{2}\right)+\pi_{16} x_{2}^{2}\left(-\frac{1}{144}+\frac{1}{72 n^{2}}\right) \\
& +x_{2}^{3}\left(\frac{2}{3} \delta_{6}+\frac{1}{3} \delta_{4}+\frac{1}{24} \delta_{2}\right) \\
& +\pi_{16} x_{2}^{3}\left(\frac{7}{36 n^{3}} L+\frac{4}{3 n^{2}} L_{8}^{r}-\frac{4}{9 n^{2}} L_{5}^{r}+\frac{20}{27 n^{2}} L_{3}^{r}+\frac{20}{27 n^{2}} L_{0}^{r}-\frac{1}{18 n} L\right. \\
& \left.-\frac{4}{9 n} L_{6}^{r}+\frac{4}{9 n} L_{4}^{r}-\frac{4}{27 n} L_{2}^{r}-\frac{4}{9 n} L_{1}^{r}-\frac{2}{9} L_{8}^{r}-\frac{2}{27} L_{3}^{r}-\frac{2}{9} L_{0}^{r}+\frac{7 n}{648} L\right) \\
& +\pi_{16}^{2} x_{2}^{3}\left(\frac{1}{12 n^{3}}+\frac{n}{324}\right)+\pi^{2} \pi_{16}^{2} x_{2}^{3}\left(\frac{1}{54 n^{3}}-\frac{1}{216 n}-\frac{n}{2592}\right), \\
& \pi a_{1}^{A S}=\pi a_{1}^{S A} \\
& \pi a_{0}^{S S}=-\frac{1}{16} x_{2} \\
& +x_{2}^{2}\left(\alpha_{3}+\frac{1}{4} \alpha_{2}+\frac{1}{16} \alpha_{1}+\beta_{4}+\frac{1}{16} \beta_{1}+\frac{1}{8} \pi_{16}\right) \\
& +x_{2}^{3}\left(\delta_{4}+\frac{1}{16} \delta_{1}+4 \gamma_{5}+\gamma_{3}+\frac{1}{4} \gamma_{2}+\frac{1}{16} \gamma_{1}\right) \\
& +\pi_{16} x_{2}^{3}\left(\frac{1}{2 n^{2}} L-\frac{1}{2 n} L+\frac{1}{2} L-4 L_{8}^{r}-8 L_{6}^{r}+4 L_{5}^{r}+8 L_{4}^{r}-4 L_{3}^{r}\right. \\
& \left.-8 L_{2}^{r}-8 L_{1}^{r}-4 L_{0}^{r}\right) \\
& +\pi_{16}^{2} x_{2}^{3}\left[-\frac{1}{2}-\frac{1}{n^{2}}+\frac{1}{n}-\frac{n}{24}+\pi^{2}\left(\frac{1}{12}+\frac{1}{6 n^{2}}-\frac{1}{6 n}+\frac{7 n}{144}\right)\right], \\
& \pi a_{0}^{A A}=+\frac{1}{16} x_{2} \\
& +x_{2}^{2}\left(-\alpha_{3}-\frac{1}{4} \alpha_{2}-\frac{1}{16} \alpha_{1}+\beta_{4}+\frac{1}{16} \beta_{1}+\frac{\pi_{16}}{8}\right) \\
& +x_{2}^{3}\left(\delta_{4}+\frac{1}{16} \delta_{1}-4 \gamma_{5}-\gamma_{3}-\frac{1}{4} \gamma_{2}-\frac{1}{16} \gamma_{1}\right) \\
& +\pi_{16} x_{2}^{3}\left(-\frac{1}{2 n^{2}} L-\frac{1}{2 n} L-\frac{1}{2} L-4 L_{8}^{r}+8 L_{6}^{r}+4 L_{5}^{r}-8 L_{4}^{r}-4 L_{3}^{r}\right. \\
& \left.+8 L_{2}^{r}+8 L_{1}^{r}-4 L_{0}^{r}\right) \\
& +\pi_{16}^{2} x_{2}^{3}\left[\frac{1}{2}+\frac{1}{n^{2}}+\frac{1}{n}-\frac{n}{24}+\pi^{2}\left(-\frac{1}{12}-\frac{1}{6 n^{2}}-\frac{1}{6 n}+\frac{7 n}{144}\right)\right] \text {. }
\end{aligned}
$$




\section{C.2 Real or adjoint case}

$$
\begin{aligned}
& \pi a_{0}^{I}=+x_{2}\left(\frac{1}{32}-\frac{1}{32 n}+\frac{n}{8}\right) \\
& +x_{2}^{2}\left(-\frac{1}{n} \alpha_{4}-\frac{1}{2 n} \alpha_{3}-\frac{1}{8 n} \alpha_{2}-\frac{3}{32 n} \alpha_{1}+\alpha_{4}+\frac{1}{2} \alpha_{3}+\frac{1}{8} \alpha_{2}+\frac{3}{32} \alpha_{1}\right. \\
& +\beta_{4}-\frac{1}{2} \beta_{3}-\frac{1}{8} \beta_{2}+\frac{1}{32} \beta_{1}+2 n \alpha_{4}+\frac{n}{8} \alpha_{1}+\frac{n}{2} \beta_{3}+\frac{n}{8} \beta_{2}+\frac{n}{32} \beta_{1} \\
& \left.+n^{2} \beta_{3}+\frac{n^{2}}{4} \beta_{2}+\frac{n^{2}}{16} \beta_{1}\right) \\
& +\pi_{16} x_{2}^{2}\left(-\frac{7}{32}+\frac{1}{32 n^{2}}-\frac{1}{16 n}+\frac{n}{4}+\frac{n^{2}}{2}\right) \\
& +x_{2}^{3}\left(-\frac{2}{n} \gamma_{5}-\frac{1}{n} \gamma_{4}-\frac{1}{2 n} \gamma_{3}-\frac{1}{8 n} \gamma_{2}-\frac{3}{32 n} \gamma_{1}-2 \delta_{5}+\delta_{4}-\frac{1}{2} \delta_{3}-\frac{1}{8} \delta_{2}\right. \\
& +\frac{1}{32} \delta_{1}+2 \gamma_{5}+\gamma_{4}+\frac{1}{2} \gamma_{3}+\frac{1}{8} \gamma_{2}+\frac{3}{32} \gamma_{1}+2 n \delta_{5}+\frac{n}{2} \delta_{3}+\frac{n}{8} \delta_{2} \\
& \left.+\frac{n}{32} \delta_{1}+2 n \gamma_{4}+\frac{n}{8} \gamma_{1}+4 n^{2} \delta_{5}+n^{2} \delta_{3}+\frac{n^{2}}{4} \delta_{2}+\frac{n^{2}}{16} \delta_{1}\right) \\
& +\pi_{16} x_{2}^{3}\left(\frac{7}{32 n^{3}} L-\frac{15}{32 n^{2}} L+\frac{3}{n^{2}} L_{8}^{r}-\frac{1}{n^{2}} L_{5}^{r}+\frac{3}{n^{2}} L_{3}^{r}+\frac{3}{n^{2}} L_{0}^{r}\right. \\
& -\frac{29}{32 n} L-\frac{6}{n} L_{8}^{r}-\frac{2}{n} L_{6}^{r}+\frac{2}{n} L_{5}^{r}+\frac{6}{n} L_{4}^{r}-\frac{6}{n} L_{3}^{r}-\frac{2}{n} L_{2}^{r}-\frac{2}{n} L_{1}^{r} \\
& -\frac{6}{n} L_{0}^{r}+\frac{55}{32} L-13 L_{8}^{r}+3 L_{5}^{r}-8 L_{4}^{r}-13 L_{3}^{r}-13 L_{0}^{r}+\frac{21 n}{16} L \\
& +16 n L_{8}^{r}+6 n L_{6}^{r}-4 n L_{5}^{r}-26 n L_{4}^{r}+16 n L_{3}^{r}+6 n L_{2}^{r}+6 n L_{1}^{r} \\
& +16 n L_{0}^{r}-\frac{19 n^{2}}{8} L+16 n^{2} L_{8}^{r}+12 n^{2} L_{6}^{r}+12 n^{2} L_{4}^{r}+16 n^{2} L_{3}^{r} \\
& +12 n^{2} L_{2}^{r}+12 n^{2} L_{1}^{r}+16 n^{2} L_{0}^{r}-\frac{5 n^{3}}{2} L+16 n^{3} L_{6}^{r}+16 n^{3} L_{4}^{r} \\
& \left.+16 n^{3} L_{2}^{r}+16 n^{3} L_{1}^{r}\right) \\
& +\pi_{16}^{2} x_{2}^{3}\left(-\frac{85}{48}-\frac{3}{8 n^{3}}+\frac{3}{4 n^{2}}+\frac{5}{4 n}-\frac{3 n}{8}+\frac{29 n^{2}}{48}+\frac{n^{3}}{12}\right) \\
& +\pi^{2} \pi_{16}^{2} x_{2}^{3}\left(\frac{89}{288}+\frac{1}{16 n^{3}}-\frac{1}{8 n^{2}}-\frac{5}{24 n}+\frac{n}{16}-\frac{49 n^{2}}{288}-\frac{5 n^{3}}{72}\right), \\
& \pi a_{1}^{A}=+x_{2}\left(\frac{1}{48}+\frac{n}{48}\right) \\
& +x_{2}^{2}\left(-\frac{1}{3} \alpha_{4}-\frac{1}{24} \alpha_{2}+\frac{1}{3} \beta_{4}+\frac{1}{24} \beta_{2}-\frac{n}{3} \alpha_{4}-\frac{n}{24} \alpha_{2}\right) \\
& +\pi_{16} x_{2}^{2}\left(-\frac{11}{864}+\frac{1}{288 n^{2}}-\frac{1}{288 n}-\frac{7 n}{864}-\frac{n^{2}}{432}\right)
\end{aligned}
$$




$$
\begin{aligned}
& +x_{2}^{3}\left(\frac{2}{3} \delta_{6}+\frac{1}{3} \delta_{4}+\frac{1}{24} \delta_{2}-\frac{2}{3} \gamma_{6}-\frac{1}{3} \gamma_{4}-\frac{1}{24} \gamma_{2}-\frac{2 n}{3} \gamma_{6}-\frac{n}{3} \gamma_{4}-\frac{n}{24} \gamma_{2}\right) \\
& +\pi_{16} x_{2}^{3}\left(\frac{7}{288 n^{3}} L-\frac{1}{36 n^{2}} L+\frac{1}{3 n^{2}} L_{8}^{r}-\frac{1}{9 n^{2}} L_{5}^{r}+\frac{5}{27 n^{2}} L_{3}^{r}+\frac{5}{27 n^{2}} L_{0}^{r}\right. \\
& -\frac{5}{288 n} L-\frac{1}{3 n} L_{8}^{r}-\frac{2}{9 n} L_{6}^{r}+\frac{1}{9 n} L_{5}^{r}+\frac{2}{9 n} L_{4}^{r}-\frac{5}{27 n} L_{3}^{r}-\frac{2}{27 n} L_{2}^{r} \\
& -\frac{2}{9 n} L_{1}^{r}-\frac{5}{27 n} L_{0}^{r}+\frac{67}{1296} L-\frac{4}{9} L_{8}^{r}-\frac{2}{9} L_{6}^{r}+\frac{1}{54} L_{5}^{r}-\frac{10}{27} L_{4}^{r} \\
& -\frac{5}{27} L_{3}^{r}-\frac{2}{9} L_{2}^{r}+\frac{2}{27} L_{1}^{r}-\frac{10}{27} L_{0}^{r}+\frac{125 n}{2592} L-\frac{4 n}{9} L_{6}^{r}-\frac{7 n}{54} L_{5}^{r} \\
& -\frac{4 n}{27} L_{4}^{r}+\frac{n}{27} L_{3}^{r}-\frac{8 n}{27} L_{2}^{r}-\frac{4 n}{27} L_{1}^{r}-\frac{2 n}{27} L_{0}^{r}+\frac{7 n^{2}}{864} L-\frac{n^{2}}{27} L_{5}^{r} \\
& \left.+\frac{n^{3}}{432} L\right) \\
& +\pi_{16}^{2} x_{2}^{3}\left(-\frac{13}{96}+\frac{1}{96 n^{3}}+\frac{7}{288 n^{2}}-\frac{281 n}{1728}-\frac{151 n^{2}}{2592}-\frac{25 n^{3}}{5184}\right) \\
& +\pi^{2} \pi_{16}^{2} x_{2}^{3}\left(\frac{173}{10368}+\frac{1}{432 n^{3}}-\frac{5}{864 n^{2}}-\frac{1}{576 n}+\frac{169 n}{10368}+\frac{n^{2}}{432}-\frac{n^{3}}{1296}\right) \text {, } \\
& \pi a_{0}^{S}=+x_{2}\left(\frac{1}{32}-\frac{1}{16 n}+\frac{n}{16}\right) \\
& +x_{2}^{2}\left(-\frac{2}{n} \alpha_{4}-\frac{1}{n} \alpha_{3}-\frac{1}{4 n} \alpha_{2}-\frac{3}{16 n} \alpha_{1}+\alpha_{4}+\frac{1}{2} \alpha_{3}+\frac{1}{8} \alpha_{2}+\frac{3}{32} \alpha_{1}\right. \\
& \left.+\beta_{4}+\frac{1}{16} \beta_{1}+n \alpha_{4}+\frac{n}{16} \alpha_{1}\right) \\
& +\pi_{16} x_{2}^{2}\left(-\frac{7}{32}+\frac{1}{8 n^{2}}-\frac{1}{8 n}+\frac{n}{8}+\frac{n^{2}}{8}\right) \\
& +x_{2}^{3}\left(-\frac{4}{n} \gamma_{5}-\frac{2}{n} \gamma_{4}-\frac{1}{n} \gamma_{3}-\frac{1}{4 n} \gamma_{2}-\frac{3}{16 n} \gamma_{1}+\delta_{4}+\frac{1}{16} \delta_{1}+2 \gamma_{5}\right. \\
& \left.+\gamma_{4}+\frac{1}{2} \gamma_{3}+\frac{1}{8} \gamma_{2}+\frac{3}{32} \gamma_{1}+n \gamma_{4}+\frac{n}{16} \gamma_{1}\right) \\
& +\pi_{16} x_{2}^{3}\left(+\frac{7}{8 n^{3}} L-\frac{19}{16 n^{2}} L+\frac{12}{n^{2}} L_{8}^{r}-\frac{4}{n^{2}} L_{5}^{r}+\frac{12}{n^{2}} L_{3}^{r}+\frac{12}{n^{2}} L_{0}^{r}-\frac{13}{16 n} L\right. \\
& -\frac{12}{n} L_{8}^{r}-\frac{8}{n} L_{6}^{r}+\frac{4}{n} L_{5}^{r}+\frac{8}{n} L_{4}^{r}-\frac{12}{n} L_{3}^{r}-\frac{8}{n} L_{2}^{r}-\frac{8}{n} L_{1}^{r}-\frac{12}{n} L_{0}^{r} \\
& +\frac{41}{32} L-13 L_{8}^{r}+4 L_{6}^{r}+3 L_{5}^{r}-4 L_{4}^{r}-13 L_{3}^{r}+4 L_{2}^{r}+4 L_{1}^{r}-13 L_{0}^{r} \\
& +\frac{3 n}{8} L+8 n L_{8}^{r}+8 n L_{6}^{r}-2 n L_{5}^{r}-8 n L_{4}^{r}+8 n L_{3}^{r}+8 n L_{2}^{r} \\
& \left.+8 n L_{1}^{r}+8 n L_{0}^{r}-\frac{n^{2}}{2} L+4 n^{2} L_{8}^{r}+4 n^{2} L_{3}^{r}+4 n^{2} L_{0}^{r}-\frac{n^{3}}{4} L\right)
\end{aligned}
$$




$$
\begin{aligned}
& +\pi_{16}^{2} x_{2}^{3}\left(-\frac{37}{48}-\frac{5}{4 n^{3}}+\frac{13}{8 n^{2}}+\frac{3}{8 n}+\frac{31 n}{48}-\frac{3 n^{2}}{16}-\frac{7 n^{3}}{24}\right) \\
& +\pi^{2} \pi_{16}^{2} x_{2}^{3}\left(\frac{41}{288}+\frac{5}{24 n^{3}}-\frac{13}{48 n^{2}}-\frac{1}{16 n}-\frac{29 n}{288}+\frac{n^{2}}{96}+\frac{5 n^{3}}{144}\right), \\
& \pi a_{0}^{F S}=+x_{2}\left(-\frac{1}{16}\right) \\
& +x_{2}^{2}\left(\alpha_{3}+\frac{1}{4} \alpha_{2}+\frac{1}{16} \alpha_{1}+\beta_{4}+\frac{1}{16} \beta_{1}\right)+\frac{1}{8} \pi_{16} x_{2}^{2} \\
& +x_{2}^{3}\left(+\delta_{4}+\frac{1}{16} \delta_{1}+4 \gamma_{5}+\gamma_{3}+\frac{1}{4} \gamma_{2}+\frac{1}{16} \gamma_{1}\right) \\
& +\pi_{16} x_{2}^{3}\left(\frac{1}{8 n^{2}} L-\frac{1}{4 n} L+\frac{3}{8} L-4 L_{8}^{r}-8 L_{6}^{r}+4 L_{5}^{r}+8 L_{4}^{r}-4 L_{3}^{r}\right. \\
& \left.-8 L_{2}^{r}-8 L_{1}^{r}-4 L_{0}^{r}\right) \\
& +\pi_{16}^{2} x_{2}^{3}\left[-\frac{17}{24}-\frac{1}{4 n^{2}}+\frac{1}{2 n}-\frac{n}{24}+\pi^{2}\left(\frac{13}{144}+\frac{1}{24 n^{2}}-\frac{1}{12 n}+\frac{7 n}{144}\right)\right] \text {, } \\
& \pi a_{1}^{M A}=+x_{2}^{2}\left[\frac{1}{3} \beta_{4}+\frac{1}{24} \beta_{2}+\pi_{16}\left(-\frac{1}{288}+\frac{1}{288 n^{2}}\right)\right] \\
& +x_{2}^{3}\left(\frac{2}{3} \delta_{6}+\frac{1}{3} \delta_{4}+\frac{1}{24} \delta_{2}\right) \\
& +\pi_{16} x_{2}^{3}\left(\frac{7}{288 n^{3}} L-\frac{1}{72 n^{2}} L+\frac{1}{3 n^{2}} L_{8}^{r}-\frac{1}{9 n^{2}} L_{5}^{r}+\frac{5}{27 n^{2}} L_{3}^{r}+\frac{5}{27 n^{2}} L_{0}^{r}\right. \\
& -\frac{1}{72 n} L-\frac{2}{9 n} L_{6}^{r}+\frac{2}{9 n} L_{4}^{r}-\frac{2}{27 n} L_{2}^{r}-\frac{2}{9 n} L_{1}^{r}+\frac{11}{2592} L-\frac{1}{9} L_{8}^{r} \\
& \left.+\frac{2}{9} L_{6}^{r}-\frac{2}{9} L_{4}^{r}-\frac{1}{27} L_{3}^{r}+\frac{2}{27} L_{2}^{r}+\frac{2}{9} L_{1}^{r}-\frac{1}{9} L_{0}^{r}+\frac{7 n}{1296} L\right) \\
& +\pi_{16}^{2} x_{2}^{3}\left(\frac{17}{2592}+\frac{1}{96 n^{3}}-\frac{1}{144 n^{2}}+\frac{n}{648}\right) \\
& +\pi^{2} \pi_{16}^{2} x_{2}^{3}\left(-\frac{1}{1296}+\frac{1}{432 n^{3}}-\frac{1}{864 n^{2}}-\frac{1}{864 n}-\frac{n}{5184}\right), \\
& \pi a_{0}^{M S}=+\frac{1}{32} x_{2} \\
& +x_{2}^{2}\left(-\frac{1}{2} \alpha_{3}-\frac{1}{8} \alpha_{2}-\frac{1}{32} \alpha_{1}+\beta_{4}+\frac{1}{16} \beta_{1}+\frac{1}{32} \pi_{16}\right) \\
& +x_{2}^{3}\left(\delta_{4}+\frac{1}{16} \delta_{1}-2 \gamma_{5}-\frac{1}{2} \gamma_{3}-\frac{1}{8} \gamma_{2}-\frac{1}{32} \gamma_{1}\right) \\
& +\pi_{16} x_{2}^{3}\left(-\frac{1}{16 n^{2}} L-\frac{1}{16 n} L-\frac{3}{32} L-L_{8}^{r}+4 L_{6}^{r}+L_{5}^{r}-4 L_{4}^{r}-L_{3}^{r}\right. \\
& \left.+4 L_{2}^{r}+4 L_{1}^{r}-L_{0}^{r}\right)
\end{aligned}
$$




$$
+\pi_{16}^{2} x_{2}^{3}\left[\frac{1}{96}+\frac{1}{8 n^{2}}+\frac{1}{8 n}-\frac{n}{96}+\pi^{2}\left(-\frac{5}{576}-\frac{1}{48 n^{2}}-\frac{1}{48 n}+\frac{7 n}{576}\right)\right] .
$$

\section{C.3 Pseudo-real or two-colour case}

$$
\begin{aligned}
& \pi a_{0}^{I}=+x_{2}\left(-\frac{1}{32}-\frac{1}{32 n}+\frac{n}{8}\right) \\
& +x_{2}^{2}\left(-\frac{1}{n} \alpha_{4}-\frac{1}{2 n} \alpha_{3}-\frac{1}{8 n} \alpha_{2}-\frac{3}{32 n} \alpha_{1}-\alpha_{4}-\frac{1}{2} \alpha_{3}-\frac{1}{8} \alpha_{2}-\frac{3}{32} \alpha_{1}\right. \\
& +\beta_{4}-\frac{1}{2} \beta_{3}-\frac{1}{8} \beta_{2}+\frac{1}{32} \beta_{1}+2 n \alpha_{4}+\frac{n}{8} \alpha_{1}-\frac{n}{2} \beta_{3}-\frac{n}{8} \beta_{2}-\frac{n}{32} \beta_{1} \\
& \left.+n^{2} \beta_{3}+\frac{n^{2}}{4} \beta_{2}+\frac{n^{2}}{16} \beta_{1}\right) \\
& +\pi_{16} x_{2}^{2}\left(-\frac{7}{32}+\frac{1}{32 n^{2}}+\frac{1}{16 n}-\frac{n}{4}+\frac{n^{2}}{2}\right) \\
& +x_{2}^{3}\left(-\frac{2}{n} \gamma_{5}-\frac{1}{n} \gamma_{4}-\frac{1}{2 n} \gamma_{3}-\frac{1}{8 n} \gamma_{2}-\frac{3}{32 n} \gamma_{1}-2 \delta_{5}+\delta_{4}-\frac{1}{2} \delta_{3}-\frac{1}{8} \delta_{2}\right. \\
& +\frac{1}{32} \delta_{1}-2 \gamma_{5}-\gamma_{4}-\frac{1}{2} \gamma_{3}-\frac{1}{8} \gamma_{2}-\frac{3}{32} \gamma_{1}-2 n \delta_{5}-\frac{n}{2} \delta_{3}-\frac{n}{8} \delta_{2} \\
& \left.-\frac{n}{32} \delta_{1}+2 n \gamma_{4}+\frac{n}{8} \gamma_{1}+4 n^{2} \delta_{5}+n^{2} \delta_{3}+\frac{n^{2}}{4} \delta_{2}+\frac{n^{2}}{16} \delta_{1}\right) \\
& +\pi_{16} x_{2}^{3}\left(\frac{7}{32 n^{3}} L+\frac{15}{32 n^{2}} L+\frac{3}{n^{2}} L_{8}^{r}-\frac{1}{n^{2}} L_{5}^{r}+\frac{3}{n^{2}} L_{3}^{r}+\frac{3}{n^{2}} L_{0}^{r}-\frac{29}{32 n} L\right. \\
& +\frac{6}{n} L_{8}^{r}-\frac{2}{n} L_{6}^{r}-\frac{2}{n} L_{5}^{r}+\frac{6}{n} L_{4}^{r}+\frac{6}{n} L_{3}^{r}-\frac{2}{n} L_{2}^{r}-\frac{2}{n} L_{1}^{r}+\frac{6}{n} L_{0}^{r} \\
& -\frac{55}{32} L-13 L_{8}^{r}+3 L_{5}^{r}+8 L_{4}^{r}-13 L_{3}^{r}-13 L_{0}^{r}+\frac{21 n}{16} L-16 n L_{8}^{r} \\
& +6 n L_{6}^{r}+4 n L_{5}^{r}-26 n L_{4}^{r}-16 n L_{3}^{r}+6 n L_{2}^{r}+6 n L_{1}^{r}-16 n L_{0}^{r} \\
& +\frac{19 n^{2}}{8} L+16 n^{2} L_{8}^{r}-12 n^{2} L_{6}^{r}-12 n^{2} L_{4}^{r}+16 n^{2} L_{3}^{r}-12 n^{2} L_{2}^{r} \\
& -12 n^{2} L_{1}^{r}+16 n^{2} L_{0}^{r}-\frac{5 n^{3}}{2} L+16 n^{3} L_{6}^{r}+16 n^{3} L_{4}^{r}+16 n^{3} L_{2}^{r} \\
& \left.+16 n^{3} L_{1}^{r}\right) \\
& +\pi_{16}^{2} x_{2}^{3}\left(\frac{85}{48}-\frac{3}{8 n^{3}}-\frac{3}{4 n^{2}}+\frac{5}{4 n}-\frac{3 n}{8}-\frac{29 n^{2}}{48}+\frac{n^{3}}{12}\right) \\
& +\pi^{2} \pi_{16}^{2} x_{2}^{3}\left(-\frac{89}{288}+\frac{1}{16 n^{3}}+\frac{1}{8 n^{2}}-\frac{5}{24 n}+\frac{n}{16}+\frac{49 n^{2}}{288}-\frac{5 n^{3}}{72}\right), \\
& \pi a_{0}^{A}=+x_{2}\left(-\frac{1}{32}-\frac{1}{16 n}+\frac{n}{16}\right) \\
& +x_{2}^{2}\left(-\frac{2}{n} \alpha_{4}-\frac{1}{n} \alpha_{3}-\frac{1}{4 n} \alpha_{2}-\frac{3}{16 n} \alpha_{1}-\alpha_{4}-\frac{1}{2} \alpha_{3}-\frac{1}{8} \alpha_{2}-\frac{3}{32} \alpha_{1}\right.
\end{aligned}
$$




$$
\begin{aligned}
& \left.+\beta_{4}+\frac{1}{16} \beta_{1}+n \alpha_{4}+\frac{n}{16} \alpha_{1}\right) \\
& +\pi_{16} x_{2}^{2}\left(-\frac{7}{32}+\frac{1}{8 n^{2}}+\frac{1}{8 n}-\frac{n}{8}+\frac{n^{2}}{8}\right) \\
& +x_{2}^{3}\left(-\frac{4}{n} \gamma_{5}-\frac{2}{n} \gamma_{4}-\frac{1}{n} \gamma_{3}-\frac{1}{4 n} \gamma_{2}-\frac{3}{16 n} \gamma_{1}+\delta_{4}+\frac{1}{16} \delta_{1}-2 \gamma_{5}-\gamma_{4}\right. \\
& \left.-\frac{1}{2} \gamma_{3}-\frac{1}{8} \gamma_{2}-\frac{3}{32} \gamma_{1}+n \gamma_{4}+\frac{n}{16} \gamma_{1}\right) \\
& +\pi_{16} x_{2}^{3}\left(\frac{7}{8 n^{3}} L+\frac{19}{16 n^{2}} L+\frac{12}{n^{2}} L_{8}^{r}-\frac{4}{n^{2}} L_{5}^{r}+\frac{12}{n^{2}} L_{3}^{r}+\frac{12}{n^{2}} L_{0}^{r}-\frac{13}{16 n} L\right. \\
& +\frac{12}{n} L_{8}^{r}-\frac{8}{n} L_{6}^{r}-\frac{4}{n} L_{5}^{r}+\frac{8}{n} L_{4}^{r}+\frac{12}{n} L_{3}^{r}-\frac{8}{n} L_{2}^{r}-\frac{8}{n} L_{1}^{r}+\frac{12}{n} L_{0}^{r} \\
& -\frac{41}{32} L-13 L_{8}^{r}-4 L_{6}^{r}+3 L_{5}^{r}+4 L_{4}^{r}-13 L_{3}^{r}-4 L_{2}^{r}-4 L_{1}^{r}-13 L_{0}^{r} \\
& +\frac{3 n}{8} L-8 n L_{8}^{r}+8 n L_{6}^{r}+2 n L_{5}^{r}-8 n L_{4}^{r}-8 n L_{3}^{r}+8 n L_{2}^{r} \\
& \left.+8 n L_{1}^{r}-8 n L_{0}^{r}+\frac{n^{2}}{2} L+4 n^{2} L_{8}^{r}+4 n^{2} L_{3}^{r}+4 n^{2} L_{0}^{r}-\frac{n^{3}}{4} L\right) \\
& +\pi_{16}^{2} x_{2}^{3}\left(\frac{37}{48}-\frac{5}{4 n^{3}}-\frac{13}{8 n^{2}}+\frac{3}{8 n}+\frac{31 n}{48}+\frac{3 n^{2}}{16}-\frac{7 n^{3}}{24}\right) \\
& +\pi^{2} \pi_{16}^{2} x_{2}^{3}\left(-\frac{41}{288}+\frac{5}{24 n^{3}}+\frac{13}{48 n^{2}}-\frac{1}{16 n}-\frac{29 n}{288}-\frac{n^{2}}{96}+\frac{5 n^{3}}{144}\right), \\
& \pi a_{1}^{S}=+x_{2}\left(-\frac{1}{48}+\frac{n}{48}\right) \\
& +x_{2}^{2}\left(\frac{1}{3} \alpha_{4}+\frac{1}{24} \alpha_{2}+\frac{1}{3} \beta_{4}+\frac{1}{24} \beta_{2}-\frac{n}{3} \alpha_{4}-\frac{n}{24} \alpha_{2}\right) \\
& +\pi_{16} x_{2}^{2}\left(-\frac{11}{864}+\frac{1}{288 n^{2}}+\frac{1}{288 n}+\frac{7 n}{864}-\frac{n^{2}}{432}\right) \\
& +x_{2}^{3}\left(\frac{2}{3} \delta_{6}+\frac{1}{3} \delta_{4}+\frac{1}{24} \delta_{2}+\frac{2}{3} \gamma_{6}+\frac{1}{3} \gamma_{4}+\frac{1}{24} \gamma_{2}-\frac{2 n}{3} \gamma_{6}-\frac{n}{3} \gamma_{4}\right. \\
& \left.-\frac{n}{24} \gamma_{2}\right) \\
& +\pi_{16} x_{2}^{3}\left(\frac{7}{288 n^{3}} L+\frac{1}{36 n^{2}} L+\frac{1}{3 n^{2}} L_{8}^{r}-\frac{1}{9 n^{2}} L_{5}^{r}+\frac{5}{27 n^{2}} L_{3}^{r}+\frac{5}{27 n^{2}} L_{0}^{r}\right. \\
& -\frac{5}{288 n} L+\frac{1}{3 n} L_{8}^{r}-\frac{2}{9 n} L_{6}^{r}-\frac{1}{9 n} L_{5}^{r}+\frac{2}{9 n} L_{4}^{r}+\frac{5}{27 n} L_{3}^{r}-\frac{2}{27 n} L_{2}^{r} \\
& -\frac{2}{9 n} L_{1}^{r}+\frac{5}{27 n} L_{0}^{r}-\frac{67}{1296} L-\frac{4}{9} L_{8}^{r}+\frac{2}{9} L_{6}^{r}+\frac{1}{54} L_{5}^{r}+\frac{10}{27} L_{4}^{r} \\
& -\frac{5}{27} L_{3}^{r}+\frac{2}{9} L_{2}^{r}-\frac{2}{27} L_{1}^{r}-\frac{10}{27} L_{0}^{r}+\frac{125 n}{2592} L-\frac{4 n}{9} L_{6}^{r}+\frac{7 n}{54} L_{5}^{r} \\
& -\frac{4 n}{27} L_{4}^{r}-\frac{n}{27} L_{3}^{r}-\frac{8 n}{27} L_{2}^{r}-\frac{4 n}{27} L_{1}^{r}+\frac{2 n}{27} L_{0}^{r}-\frac{7 n^{2}}{864} L-\frac{n^{2}}{27} L_{5}^{r}
\end{aligned}
$$




$$
\begin{aligned}
& \left.+\frac{n^{3}}{432} L\right) \\
& +\pi_{16}^{2} x_{2}^{3}\left(\frac{13}{96}+\frac{1}{96 n^{3}}-\frac{7}{288 n^{2}}-\frac{281 n}{1728}+\frac{151 n^{2}}{2592}-\frac{25 n^{3}}{5184}\right) \\
& +\pi^{2} \pi_{16}^{2} x_{2}^{3}\left(-\frac{173}{10368}+\frac{1}{432 n^{3}}+\frac{5}{864 n^{2}}-\frac{1}{576 n}+\frac{169 n}{10368}-\frac{n^{2}}{432}\right. \\
& \left.-\frac{n^{3}}{1296}\right) \\
& \pi a_{0}^{F A}=+\frac{1}{16} x_{2} \\
& +x_{2}^{2}\left(-\alpha_{3}-\frac{1}{4} \alpha_{2}-\frac{1}{16} \alpha_{1}+\beta_{4}+\frac{1}{16} \beta_{1}+\frac{1}{8} \pi_{16}\right) \\
& +x_{2}^{3}\left(\delta_{4}+\frac{1}{16} \delta_{1}-4 \gamma_{5}-\gamma_{3}-\frac{1}{4} \gamma_{2}-\frac{1}{16} \gamma_{1}\right) \\
& +\pi_{16} x_{2}^{3}\left(-\frac{1}{8 n^{2}} L-\frac{1}{4 n} L-\frac{3}{8} L-4 L_{8}^{r}+8 L_{6}^{r}+4 L_{5}^{r}-8 L_{4}^{r}-4 L_{3}^{r}\right. \\
& \left.+8 L_{2}^{r}+8 L_{1}^{r}-4 L_{0}^{r}\right) \\
& +\pi_{16}^{2} x_{2}^{3}\left[\frac{17}{24}+\frac{1}{4 n^{2}}+\frac{1}{2 n}-\frac{n}{24}+\pi^{2}\left(\frac{7 n}{144}-\frac{13}{144}-\frac{1}{24 n^{2}}-\frac{1}{12 n}\right)\right] \text {, } \\
& \pi a_{1}^{M A}=+x_{2}^{2}\left[\frac{1}{3} \beta_{4}+\frac{1}{24} \beta_{2}+\pi_{16}\left(-\frac{1}{288}+\frac{1}{288 n^{2}}\right)\right] \\
& +x_{2}^{3}\left(\frac{2}{3} \delta_{6}+\frac{1}{3} \delta_{4}+\frac{1}{24} \delta_{2}\right) \\
& +\pi_{16} x_{2}^{3}\left(\frac{7}{288 n^{3}} L+\frac{1}{72 n^{2}} L+\frac{1}{3 n^{2}} L_{8}^{r}-\frac{1}{9 n^{2}} L_{5}^{r}+\frac{5}{27 n^{2}} L_{3}^{r}+\frac{5}{27 n^{2}} L_{0}^{r}\right. \\
& -\frac{1}{72 n} L-\frac{2}{9 n} L_{6}^{r}+\frac{2}{9 n} L_{4}^{r}-\frac{2}{27 n} L_{2}^{r}-\frac{2}{9 n} L_{1}^{r}-\frac{11}{2592} L-\frac{1}{9} L_{8}^{r} \\
& \left.-\frac{2}{9} L_{6}^{r}+\frac{2}{9} L_{4}^{r}-\frac{1}{27} L_{3}^{r}-\frac{2}{27} L_{2}^{r}-\frac{2}{9} L_{1}^{r}-\frac{1}{9} L_{0}^{r}+\frac{7 n}{1296} L\right) \\
& +\pi_{16}^{2} x_{2}^{3}\left(-\frac{17}{2592}+\frac{1}{96 n^{3}}+\frac{1}{144 n^{2}}+\frac{n}{648}\right) \\
& +\pi^{2} \pi_{16}^{2} x_{2}^{3}\left(\frac{1}{1296}+\frac{1}{432 n^{3}}+\frac{1}{864 n^{2}}-\frac{1}{864 n}-\frac{n}{5184}\right), \\
& \pi a_{0}^{M S}=-\frac{1}{32} x_{2} \\
& +x_{2}^{2}\left(\frac{1}{2} \alpha_{3}+\frac{1}{8} \alpha_{2}+\frac{1}{32} \alpha_{1}+\beta_{4}+\frac{1}{16} \beta_{1}+\frac{1}{32} \pi_{16}\right) \\
& +x_{2}^{3}\left(+\delta_{4}+\frac{1}{16} \delta_{1}+2 \gamma_{5}+\frac{1}{2} \gamma_{3}+\frac{1}{8} \gamma_{2}+\frac{1}{32} \gamma_{1}\right)
\end{aligned}
$$




$$
\begin{aligned}
& +\pi_{16} x_{2}^{3}\left(+\frac{1}{16 n^{2}} L-\frac{1}{16 n} L+\frac{3}{32} L-L_{8}^{r}-4 L_{6}^{r}+L_{5}^{r}+4 L_{4}^{r}-L_{3}^{r}\right. \\
& \left.-4 L_{2}^{r}-4 L_{1}^{r}-L_{0}^{r}\right) \\
& +\pi_{16}^{2} x_{2}^{3}\left[\frac{1}{8 n}-\frac{1}{96}-\frac{1}{8 n^{2}}-\frac{11 n}{96}+\pi^{2}\left(\frac{5}{576}+\frac{1}{48 n^{2}}-\frac{1}{48 n}+\frac{7 n}{576}\right)\right] .
\end{aligned}
$$

\section{Loop integrals}

\section{D.1 One-loop integrals}

We use dimensional regularization here throughout in $d$ dimensions with $d=4-2 \epsilon$. We need integrals with one, two and three propagators in principle. These one propagator integral is

$$
A\left(m^{2}\right)=\frac{1}{i} \int \frac{d^{d} q}{(2 \pi)^{d}} \frac{1}{q^{2}-m^{2}} .
$$

The two propagator integrals we encountered are

$$
\begin{aligned}
B\left(m_{1}^{2}, m_{2}^{2}, p^{2}\right) & =\frac{1}{i} \int \frac{d^{d} q}{(2 \pi)^{d}} \frac{1}{\left(q^{2}-m_{1}^{2}\right)\left((q-p)^{2}-m_{2}^{2}\right)} \\
B_{\mu}\left(m_{1}^{2}, m_{2}^{2}, p\right) & =\frac{1}{i} \int \frac{d^{d} q}{(2 \pi)^{d}} \frac{q_{\mu}}{\left(q^{2}-m_{1}^{2}\right)\left((q-p)^{2}-m_{2}^{2}\right)} \\
& =p_{\mu} B_{1}\left(m_{1}^{2}, m_{2}^{2}, p^{2}\right), \\
B_{\mu \nu}\left(m_{1}^{2}, m_{2}^{2}, p\right) & =\frac{1}{i} \int \frac{d^{d} q}{(2 \pi)^{d}} \frac{q_{\mu} q_{\nu}}{\left(q^{2}-m_{1}^{2}\right)\left((q-p)^{2}-m_{2}^{2}\right)} \\
& =p_{\mu} p_{\nu} B_{21}\left(m_{1}^{2}, m_{2}^{2}, p^{2}\right)+g_{\mu \nu} B_{22}\left(m_{1}^{2}, m_{2}^{2}, p^{2}\right) .
\end{aligned}
$$

All the cases with three propagator integrals that show up can be absorbed into the two-propagator ones by moving to the real masses rather than the lowest order masses. This provides a consistency check on the calculations.

The explicit expressions are well known

$$
\begin{aligned}
A\left(m^{2}\right)= & \frac{m^{2}}{16 \pi^{2}}\left\{\lambda_{0}-\ln \left(m^{2}\right)+\epsilon\left(\frac{C^{2}}{2}+\frac{1}{2}+\frac{\pi^{2}}{12}+\frac{1}{2} \ln ^{2}\left(m^{2}\right)\right.\right. \\
& \left.\left.-C \ln \left(m^{2}\right)\right)\right\}+\mathcal{O}\left(\epsilon^{2}\right) \\
B\left(m_{1}^{2}, m_{2}^{2}, p^{2}\right)= & \frac{1}{16 \pi^{2}}\left(\lambda_{0}-\frac{m_{1}^{2} \ln \left(m_{1}^{2}\right)-m_{2}^{2} \ln \left(m_{2}^{2}\right)}{m_{1}^{2}-m_{2}^{2}}\right)+\bar{J}\left(m_{1}^{2}, m_{2}^{2}, p^{2}\right)+\mathcal{O}(\epsilon), \\
\bar{J}\left(m_{1}^{2}, m_{2}^{2}, p^{2}\right)= & -\frac{1}{16 \pi^{2}} \int_{0}^{1} d x \ln \left(\frac{m_{1}^{2} x+m_{2}^{2}(1-x)-x(1-x) p^{2}}{m_{1}^{2} x+m_{2}^{2}(1-x)}\right)
\end{aligned}
$$

$C=\ln (4 \pi)+1-\gamma$ and $\lambda_{0}=1 / \epsilon+C$. The function $\bar{J}\left(m_{1}^{2}, m_{2}^{2}, p^{2}\right)$ develops an imaginary part for $p^{2} \geq\left(m_{1}+m_{2}\right)^{2}$. Using $\Delta=m_{1}^{2}-m_{2}^{2}, \Sigma=m_{1}^{2}+m_{2}^{2}$ and $\nu^{2}=p^{4}+m_{1}^{4}+m_{2}^{4}-2 p^{2} m_{1}^{2}-2 p^{2} m_{2}^{2}-2 m_{1}^{2} m_{2}^{2}$ it is given by

$$
\left(32 \pi^{2}\right) \bar{J}\left(m_{1}^{2}, m_{2}^{2}, p^{2}\right)=2+\left(-\frac{\Delta}{p^{2}}+\frac{\Sigma}{\Delta}\right) \ln \frac{m_{1}^{2}}{m_{2}^{2}}-\frac{\nu}{p^{2}} \ln \frac{\left(p^{2}+\nu\right)^{2}-\Delta^{2}}{\left(p^{2}-\nu\right)^{2}-\Delta^{2}} .
$$


The two-propagator integrals can all be reduced to $B$ and $A$ via

$$
\begin{aligned}
B_{1}\left(m_{1}^{2}, m_{2}^{2}, p^{2}\right)= & -\frac{1}{2 p^{2}}\left(A\left(m_{1}^{2}\right)-A\left(m_{2}^{2}\right)+\left(m_{2}^{2}-m_{1}^{2}-p^{2}\right) B\left(m_{1}^{2}, m_{2}^{2}, p^{2}\right)\right) \\
B_{22}\left(m_{1}^{2}, m_{2}^{2}, p^{2}\right)= & \frac{1}{2(d-1)}\left(A\left(m_{2}^{2}\right)+2 m_{1}^{2} B\left(m_{1}^{2}, m_{2}^{2}, p^{2}\right)\right. \\
& \left.+\left(m_{2}^{2}-m_{1}^{2}-p^{2}\right) B_{1}\left(m_{1}^{2}, m_{2}^{2}, p^{2}\right)\right) \\
B_{21}\left(m_{1}^{2}, m_{2}^{2}, p^{2}\right)= & \frac{1}{p^{2}}\left(A\left(m_{2}^{2}\right)+m_{1}^{2} B\left(m_{1}^{2}, m_{2}^{2}, p^{2}\right)-d B_{22}\left(m_{1}^{2}, m_{2}^{2}, p^{2}\right)\right)
\end{aligned}
$$

The basic method used here is the one from Passarino and Veltman [33].

\section{D.2 Sunset integrals}

Sunset integrals have been treated in many places already, in general and for various special cases. We use here a method that is a hybrid of various other approaches. We only cite the literature actually used. We define

$$
\begin{aligned}
\langle\langle X\rangle\rangle=\frac{1}{i^{2}} \int \frac{d^{d} q}{(2 \pi)^{d}} & \frac{d^{d} r}{(2 \pi)^{d}} \frac{X}{\left(q^{2}-m_{1}^{2}\right)\left(r^{2}-m_{2}^{2}\right)\left((q+r-p)^{2}-m_{3}^{2}\right)}, \\
H\left(m_{1}^{2}, m_{2}^{2}, m_{3}^{2} ; p^{2}\right) & =\langle\langle 1\rangle\rangle \\
H_{\mu}\left(m_{1}^{2}, m_{2}^{2}, m_{3}^{2} ; p^{2}\right) & =\left\langle\left\langle q_{\mu}\right\rangle\right\rangle=p_{\mu} H_{1}\left(m_{1}^{2}, m_{2}^{2}, m_{3}^{2} ; p^{2}\right) \\
H_{\mu \nu}\left(m_{1}^{2}, m_{2}^{2}, m_{3}^{2} ; p^{2}\right) & =\left\langle\left\langle q_{\mu} q_{\nu}\right\rangle\right\rangle \\
& =p_{\mu} p_{\nu} H_{21}\left(m_{1}^{2}, m_{2}^{2}, m_{3}^{2} ; p^{2}\right)+g_{\mu \nu} H_{22}\left(m_{1}^{2}, m_{2}^{2}, m_{3}^{2} ; p^{2}\right) .
\end{aligned}
$$

By redefining momenta the others can be simply related to the above three:

$$
\begin{aligned}
\left\langle\left\langle r_{\mu}\right\rangle\right\rangle & =p_{\mu} H_{1}\left(m_{2}^{2}, m_{1}^{2}, m_{3}^{2} ; p^{2}\right), \\
\left\langle\left\langle r_{\mu} r_{\nu}\right\rangle\right\rangle & =p_{\mu} p_{\nu} H_{21}\left(m_{2}^{2}, m_{1}^{2}, m_{3}^{2} ; p^{2}\right)+g_{\mu \nu} H_{22}\left(m_{2}^{2}, m_{1}^{2}, m_{3}^{2} ; p^{2}\right), \\
\left\langle\left\langle q_{\mu} r_{\nu}\right\rangle\right\rangle & =\left\langle\left\langle r_{\mu} q_{\nu}\right\rangle\right\rangle, \\
\left\langle\left\langle q_{\mu} r_{\nu}\right\rangle\right\rangle & =p_{\mu} p_{\nu} H_{23}\left(m_{1}^{2}, m_{2}^{2}, m_{3}^{2} ; p^{2}\right)+g_{\mu \nu} H_{24}\left(m_{1}^{2}, m_{2}^{2}, m_{3}^{2} ; p^{2}\right),
\end{aligned}
$$

with

$$
\begin{aligned}
2 H_{23}\left(m_{1}^{2}, m_{2}^{2}, m_{3}^{2} ; p^{2}\right)= & -H_{21}\left(m_{1}^{2}, m_{2}^{2}, m_{3}^{2} ; p^{2}\right)-H_{21}\left(m_{2}^{2}, m_{1}^{2}, m_{3}^{2} ; p^{2}\right) \\
& +H_{21}\left(m_{3}^{2}, m_{1}^{2}, m_{2}^{2} ; p^{2}\right)+2 H_{1}\left(m_{1}^{2}, m_{2}^{2}, m_{3}^{2} ; p^{2}\right) \\
& +2 H_{1}\left(m_{2}^{2}, m_{1}^{2}, m_{3}^{2} ; p^{2}\right)-H\left(m_{1}^{2}, m_{2}^{2}, m_{3}^{2} ; p^{2}\right), \\
2 H_{24}\left(m_{1}^{2}, m_{2}^{2}, m_{3}^{2} ; p^{2}\right)= & -H_{22}\left(m_{1}^{2}, m_{2}^{2}, m_{3}^{2} ; p^{2}\right)-H_{22}\left(m_{2}^{2}, m_{1}^{2}, m_{3}^{2} ; p^{2}\right) \\
& +H_{22}\left(m_{3}^{2}, m_{1}^{2}, m_{2}^{2} ; p^{2}\right) .
\end{aligned}
$$

The first two follow from interchanging $q$ and $r$ and the third from the fact that it is proportional to $g_{\mu \nu}$ or $p_{\mu} p_{\nu}$, which are both symmetric in $\mu$ and $\nu$. The last one is deribed using

$$
\begin{aligned}
\left(q_{\mu} r_{\nu}+r_{\mu} q_{\nu}\right)= & \left(q_{\mu}+r_{\mu}-p_{\mu}\right)\left(q_{\nu}+r_{\nu}-p_{\nu}\right)-q_{\mu} q_{\nu}-r_{\mu} r_{\nu}-p_{\mu} p_{\nu} \\
& +2 p_{\mu}\left(q_{\nu}+r_{\nu}\right)+2 p_{\nu}\left(q_{\mu}+r_{\mu}\right)
\end{aligned}
$$


and redefining momenta and masses on the r.h.s.. In addition we have the relation

$$
\begin{gathered}
p^{2} H_{21}\left(m_{1}^{2}, m_{2}^{2}, m_{3}^{2} ; p^{2}\right)+d H_{22}\left(m_{1}^{2}, m_{2}^{2}, m_{3}^{2} ; p^{2}\right)= \\
m_{1}^{2} H\left(m_{1}^{2}, m_{2}^{2}, m_{3}^{2} ; p^{2}\right)+A\left(m_{2}^{2}\right) A\left(m_{3}^{2}\right) .
\end{gathered}
$$

which allows to express $H_{22}$ in a simple way in terms of $H_{21}$. There is also a relation between $H_{1}$ and $H$

$$
\begin{array}{r}
H_{1}\left(m_{1}^{2}, m_{2}^{2}, m_{3}^{2} ; p^{2}\right)+H_{1}\left(m_{2}^{2}, m_{1}^{2}, m_{3}^{2} ; p^{2}\right)+H_{1}\left(m_{3}^{2}, m_{1}^{2}, m_{2}^{2} ; p^{2}\right)= \\
H\left(m_{1}^{2}, m_{2}^{2}, m_{3}^{2} ; p^{2}\right),
\end{array}
$$

which allows to write $H_{1}\left(m^{2}, m^{2}, m^{2} ; p^{2}\right)=1 / 3 H\left(m^{2}, m^{2}, m^{2} ; p^{2}\right)$ in the case of equal masses. The function $H$ is fully symmetric in $m_{1}^{2}, m_{2}^{2}$ and $m_{3}^{2}$, while $H_{1}, H_{21}$ and $H_{22}$ are symmetric under the interchange of $m_{2}^{2}$ and $m_{3}^{2}$.

We only need the sunset integrals at $p^{2}=m_{1}^{2}=m_{2}^{2}=m_{3}^{2}$ and their derivatives w.r.t. $p^{2}$. These have been calculated using the methods of [29]. With $H_{i d}=\frac{\partial}{\partial p^{2}} H_{i}$ we obtain

$$
\begin{aligned}
H= & \lambda_{1} m^{2}\left(\frac{5 \pi_{16}^{2}}{4}-3 L \pi_{16}\right)+\frac{3}{2} \lambda_{2} m^{2} \pi_{16}^{2} \\
& +m^{2}\left(3 L^{2}-\frac{5}{2} L \pi_{16}+\frac{1}{4} \pi^{2} \pi_{16}^{2}+\frac{15 \pi_{16}^{2}}{8}\right) \\
H_{21}= & \lambda_{1} m^{2}\left(\frac{11 \pi_{16}^{2}}{72}-\frac{2}{3} L \pi_{16}\right)+\frac{1}{3} \lambda_{2} m^{2} \pi_{16}^{2} \\
& +m^{2}\left(-\frac{11}{36} L \pi_{16}+\frac{1}{18} \pi^{2} \pi_{16}^{2}+\frac{493 \pi_{16}^{2}}{864}\right) \\
H_{22}= & \lambda_{1} m^{4}\left(\frac{157 \pi_{16}^{2}}{288}-\frac{13}{12} L \pi_{16}\right)+\frac{13}{24} \lambda_{2} m^{4} \pi_{16}^{2} \\
& +m^{4}\left(-\frac{157}{144} L \pi_{16}+\frac{13 L^{2} 4}{12}+\frac{13}{144} \pi^{2} \pi_{16}^{2}+\frac{2933 \pi_{16}^{2}}{3456}\right) \\
H_{d}= & \frac{L \pi_{16}}{2}-\frac{\lambda_{1} \pi_{16}^{2}}{4}+\frac{7 \pi_{16}^{2}}{8} \\
H_{21 d}= & \frac{L \pi_{16}}{12}-\frac{\lambda_{1} \pi_{16}^{2}}{24}+\frac{43 \pi_{16}^{2}}{288} \\
H_{22 d}= & \frac{5}{48} L m^{2} \pi_{16}-\frac{5}{96} \lambda_{1} m^{2} \pi_{16}^{2}+\frac{179 \pi_{16}^{2}}{1152} m^{2}
\end{aligned}
$$

$H_{1}$ and $H_{1 d}$ follow immmediately using (127).

\section{D.3 Vertex integrals}

The vertex diagram (16) in Fig. 2 is the most difficult two-loop diagram in $\phi \phi$ scattering, and it can also appear in other process. The two loop integral for the equal mass case can be written as

$$
\langle\langle X\rangle\rangle=\frac{\mu^{4 \epsilon}}{i^{2}} \iint d r d s \frac{X}{\left(r^{2}-m^{2}\right) \cdot\left[(r-q)^{2}-m^{2}\right] \cdot\left(s^{2}-m^{2}\right) \cdot\left[(s+r-p)^{2}-m^{2}\right]}
$$


The Lorentz decompositions of the vertex integrals are [34]

$$
\begin{aligned}
\langle\langle 1\rangle\rangle= & V, \\
\left\langle\left\langle r_{\mu}\right\rangle\right\rangle= & p_{\mu} V_{11}+q_{\mu} V_{12}, \\
\left\langle\left\langle s_{\mu}\right\rangle\right\rangle= & p_{\mu} V_{13}+q_{\mu} V_{14}, \\
\left\langle\left\langle r_{\mu} r_{\nu}\right\rangle\right\rangle= & g_{\mu \nu} V_{21}+p_{\mu} p_{\nu} V_{22}+q_{\mu} q_{\nu} V_{23}+\left(p_{\mu} q_{\nu}+q_{\mu} p_{\nu}\right) V_{24}, \\
\left\langle\left\langle r_{\mu} s_{\nu}\right\rangle\right\rangle= & g_{\mu \nu} V_{25}+p_{\mu} p_{\nu} V_{26}+q_{\mu} q_{\nu} V_{27}+q_{\mu} p_{\nu} V_{28}+p_{\mu} q_{\nu} V_{29}, \\
\left\langle\left\langle s_{\mu} s_{\nu}\right\rangle\right\rangle= & g_{\mu \nu} V_{210}+p_{\mu} p_{\nu} V_{211}+q_{\mu} q_{\nu} V_{212}+\left(q_{\mu} p_{\nu}+p_{\mu} q_{\nu}\right) V_{213}, \\
\left\langle\left\langle r_{\mu} r_{\nu} r_{\alpha}\right\rangle\right\rangle= & \left(g_{\mu \nu} p_{\alpha}+g_{\mu \alpha} p_{\nu}+g_{\nu \alpha} p_{\mu}\right) V_{31}+\left(g_{\mu \nu} q_{\alpha}+g_{\mu \alpha} q_{\nu}+g_{\nu \alpha} q_{\mu}\right) V_{32} \\
& +p_{\mu} p_{\nu} p_{\alpha} V_{33}+q_{\mu} q_{\nu} q_{\alpha} V_{34} \\
& +\left(p_{\mu} p_{\nu} q_{\alpha}+p_{\mu} q_{\nu} p_{\alpha}+q_{\mu} p_{\nu} p_{\alpha}\right) V_{35}+\left(q_{\mu} q_{\nu} p_{\alpha}+q_{\mu} p_{\nu} q_{\alpha}+p_{\mu} q_{\nu} q_{\alpha}\right) V_{36}, \\
\left\langle\left\langle r_{\mu} r_{\nu} s_{\alpha}\right\rangle\right\rangle= & g_{\mu \nu} p_{\alpha} V_{37}+g_{\mu \nu} q_{\alpha} V_{38}+\left(g_{\mu \alpha} p_{\nu}+g_{\nu \alpha} p_{\mu}\right) V_{39}+\left(g_{\mu \alpha} q_{\nu}+g_{\nu \alpha} q_{\mu}\right) V_{310} \\
& +p_{\mu} p_{\nu} p_{\alpha} V_{311}+q_{\mu} q_{\nu} q_{\alpha} V_{312}+p_{\mu} p_{\nu} q_{\alpha} V_{313}+q_{\mu} q_{\nu} p_{\alpha} V_{314} \\
& +\left(p_{\mu} q_{\nu}+q_{\mu} p_{\nu}\right) p_{\alpha} V_{315}+\left(p_{\mu} q_{\nu}+q_{\mu} p_{\nu}\right) q_{\alpha} V_{316}, \\
\left\langle\left\langle r_{\mu} s_{\nu} s_{\alpha}\right\rangle\right\rangle= & p_{\mu} g_{\nu \alpha} V_{317}+q_{\mu} g_{\nu \alpha} V_{318}+\left(g_{\mu \nu} p_{\alpha}+g_{\mu \alpha} p_{\nu}\right) V_{319}+\left(g_{\mu \nu} q_{\alpha}+g_{\mu \alpha} q_{\nu}\right) V_{320} \\
& +p_{\mu} p_{\nu} p_{\alpha} V_{321}+q_{\mu} q_{\nu} q_{\alpha} V_{322}+p_{\mu} q_{\nu} q_{\alpha} V_{323}+q_{\mu} p_{\nu} p_{\alpha} V_{324} \\
& +p_{\mu}\left(p_{\nu} q_{\alpha}+q_{\nu} p_{\alpha}\right) V_{325}+q_{\mu}\left(p_{\nu} q_{\alpha}+q_{\nu} p_{\alpha}\right) V_{326} .
\end{aligned}
$$

The $\left\langle\left\langle s_{\mu} s_{\nu} s_{\alpha}\right\rangle\right\rangle$ does not show up in $\phi \phi$ scattering. Most of those $V_{i}$ functions have been calculated analytically in [20] except the $\left\langle\left\langle s_{\mu} s_{\nu}\right\rangle\right\rangle$ and $\left\langle\left\langle r_{\mu} s_{\nu} s_{\alpha}\right\rangle\right\rangle$. We have calculated the rest of them in this work, which are $V_{210}-V_{213}$ and $V_{317}-V_{326}$. Again, the methods of [29] were used here, somewhat extended to the cases at hand. We have compared our results with the numerical evaluation for general masses described in [34]. The quantity $B^{\epsilon}$ is the next term in the expansion of $B$ in (118) but these terms always cancel in the final result.

$$
\begin{aligned}
V_{0}= & \lambda_{1}\left(\pi_{16} \bar{B}+\frac{\pi_{16}^{2}}{2}\right)+\lambda_{2} \frac{\pi_{16}^{2}}{2}+\pi_{16} B^{\epsilon} \\
& +\bar{J}\left(2 \pi_{16}-L\right)+\frac{k_{1}}{2}-\frac{k_{3}}{3}+\frac{L^{2}}{2}-\pi_{16}^{2} \\
V_{11}= & \lambda_{1} \frac{\pi_{16}^{2}}{4}+\frac{1}{2} \pi_{16} \bar{J}-\frac{1}{3} k_{3}-2 k_{4}-\frac{L \pi_{16}}{2}-\frac{7 \pi_{16}^{2}}{8} \\
V_{12}= & \lambda_{1}\left(\frac{1}{2} \pi_{16} \bar{B}+\frac{\pi_{16}^{2}}{8}\right)+\lambda_{2} \frac{\pi_{16}^{2}}{4}+\frac{1}{2} \pi_{16} B^{\epsilon} \\
& +\bar{J}\left(\frac{3 \pi_{16}}{4}-\frac{L}{2}\right)+\frac{k_{1}}{4}+k_{4}+\frac{L^{2}}{4}+\frac{L \pi_{16}}{4}-\frac{\pi_{16}^{2}}{16} \\
V_{13}= & \lambda_{1}\left(\frac{1}{2} \pi_{16} \bar{B}+\frac{\pi_{16}^{2}}{8}\right)+\lambda_{2} \frac{\pi_{16}^{2}}{4}+\frac{1}{2} \pi_{16} B^{\epsilon} \\
& +\bar{J}\left(\frac{3 \pi_{16}}{4}-\frac{L}{2}\right)+\frac{k_{1}}{4}+k_{4}+\frac{L^{2}}{4}+\frac{L \pi_{16}}{4}-\frac{\pi_{16}^{2}}{16} \\
V_{14}= & -\lambda_{1}\left(\frac{1}{4} \pi_{16} \bar{B}+\frac{\pi_{16}^{2}}{16}\right)-\lambda_{2} \frac{\pi_{16}^{2}}{8}-\frac{1}{4} \pi_{16} B^{\epsilon}
\end{aligned}
$$




$$
\begin{aligned}
& +\bar{J}\left(\frac{L}{4}-\frac{3 \pi_{16}}{8}\right)-\frac{k_{1}}{8}-\frac{k_{4}}{2}-\frac{L^{2}}{8}-\frac{L \pi_{16}}{8}+\frac{\pi_{16}^{2}}{32} \\
& V_{21}=\frac{1}{2} \lambda_{1}\left\{\pi_{16}\left[s \bar{B}_{21}+\bar{B}\left(m^{2}-\frac{1}{2} s\right)-L m^{2}\right]+\pi_{16}^{2}\left(\frac{11 m^{2}}{6}-\frac{13 s}{72}\right)\right\} \\
& +\frac{1}{2} \lambda_{2} \pi_{16}^{2}\left(m^{2}-\frac{s}{12}\right)+B^{\epsilon} \pi_{16}\left(\frac{m^{2}}{3}-\frac{s}{12}\right) \\
& +\bar{J}\left(\frac{L s}{12}-\frac{L m^{2}}{3}+\frac{5 m^{2} \pi_{16}}{12}-\frac{5 \pi_{16} s}{24}\right)-\frac{m^{2}}{6} k_{1}-\frac{s}{24} k_{2}-\frac{m^{2}}{6} k_{3}-m^{2} k_{4} \\
& +\frac{5 L^{2} m^{2}}{6}-\frac{L^{2} s}{24}+\pi_{16} L\left(\frac{s}{24}-\frac{7 m^{2}}{6}\right)+\pi_{16}^{2}\left(\frac{m^{2} \pi^{2}}{18}+\frac{11 m^{2}}{24}-\frac{\pi^{2} s}{72}-\frac{5 s}{96}\right) \\
& V_{22}=\lambda_{1} \frac{\pi_{16}^{2}}{12}+\frac{1}{6} \pi_{16} \bar{J}-\frac{k_{3}}{3}-6 k_{4}-12 k_{5}-\frac{L \pi_{16}}{6}-\frac{7 \pi_{16}^{2}}{24} \\
& V_{23}=\lambda_{1}\left(\pi_{16} \bar{B}_{21}+\frac{\pi_{16}^{2}}{36}\right)+\lambda_{2} \frac{\pi_{16}^{2}}{6}+B^{\epsilon} \pi_{16}\left(\frac{1}{3}-\frac{m^{2}}{3 s}\right) \\
& +\bar{J}\left(\frac{L m^{2}}{3 s}-\frac{L}{3}-\frac{2 m^{2} \pi_{16}}{3 s}+\frac{\pi_{16}}{2}\right)+\frac{3 k_{1}}{16}-\frac{k_{2}}{48}-\frac{k_{4}}{4}-3 k_{5}+\frac{k_{9}}{12} \\
& +\frac{L^{2} m^{2}}{6 s}+\frac{L^{2}}{6}+\pi_{16} L\left(\frac{m^{2}}{3 s}+\frac{1}{6}\right)+\pi_{16}^{2}\left(\frac{m^{2} \pi^{2}}{36 s}+\frac{m^{2}}{6 s}+\frac{5}{24}\right) \\
& V_{24}=\lambda_{1} \frac{\pi_{16}^{2}}{12}+\frac{1}{6} \pi_{16} \bar{J}+2 k_{4}+6 k_{5}-\frac{L \pi_{16}}{6}-\frac{7 \pi_{16}^{2}}{24} \\
& V_{25}=\frac{1}{4} \lambda_{1}\left\{\pi_{16}\left[-s \bar{B}_{21}+\bar{B}\left(\frac{s}{2}-m^{2}\right)+L m^{2}\right]+\pi_{16}^{2}\left(\frac{13 s}{72}-\frac{11}{6} m^{2}\right)\right\} \\
& +\frac{1}{4} \lambda_{2} \pi_{16}^{2}\left(\frac{s}{12}-m^{2}\right)+B^{\epsilon} \pi_{16}\left(\frac{s}{24}-\frac{m^{2}}{6}\right) \\
& +\bar{J}\left(\frac{L m^{2}}{6}-\frac{L s}{24}-\frac{5 m^{2} \pi_{16}}{24}+\frac{5 \pi_{16} s}{48}\right)+\frac{m^{2}}{12} k_{1}+\frac{s}{48} k_{2}+\frac{m^{2}}{12} k_{3}+\frac{m^{2}}{2} k_{4} \\
& -\frac{5 L^{2} m^{2}}{12}+\frac{L^{2} s}{48}+L \pi_{16}\left(\frac{7 m^{2}}{12}-\frac{s}{48}\right)+\pi_{16}^{2}\left(\frac{\pi^{2} s}{144}+\frac{5 s}{192}-\frac{m^{2} \pi^{2}}{36}-\frac{11 m^{2}}{48}\right) \\
& V_{26}=\lambda_{1} \frac{\pi_{16}^{2}}{12}+\frac{1}{6} \pi_{16} \bar{J}+2 k_{4}+6 k_{5}-\frac{L \pi_{16}}{6}-\frac{7 \pi_{16}^{2}}{24} \\
& V_{27}=-\frac{1}{2} \lambda_{1}\left(\pi_{16} \bar{B}_{21}+\frac{\pi_{16}^{2}}{36}\right)-\lambda_{2} \frac{\pi_{16}^{2}}{12}+B^{\epsilon} \pi_{16}\left(\frac{m^{2}}{6 s}-\frac{1}{6}\right) \\
& +\bar{J}\left(\frac{L}{6}-\frac{L m^{2}}{6 s}+\frac{m^{2} \pi_{16}}{3 s}-\frac{\pi_{16}}{4}\right)-\frac{3 k_{1}}{32}+\frac{k_{2}}{96}+\frac{k_{4}}{8}+\frac{3 k_{5}}{2}-\frac{k_{9}}{24} \\
& -\frac{L^{2} m^{2}}{12 s}-\frac{L^{2}}{12}-L \pi_{16}\left(\frac{m^{2}}{6 s}+\frac{1}{12}\right)-\pi_{16}^{2}\left(\frac{m^{2} \pi^{2}}{72 s}+\frac{m^{2}}{12 s}+\frac{5}{48}\right) \\
& V_{28}=\frac{1}{4} \lambda_{1}\left(\pi_{16} \bar{B}+\frac{\pi_{16}^{2}}{12}\right)+\lambda_{2} \frac{\pi_{16}^{2}}{8}+\frac{1}{4} \pi_{16} B^{\epsilon} \\
& +\bar{J}\left(\frac{7 \pi_{16}}{24}-\frac{L}{4}\right)+\frac{k_{1}}{8}-\frac{k_{4}}{2}-3 k_{5}+\frac{L^{2}}{8}+\frac{5 L \pi_{16}}{24}+\frac{11 \pi_{16}^{2}}{96} \\
& V_{29}=-\lambda_{1} \frac{\pi_{16}^{2}}{24}-\frac{1}{12} \pi_{16} \bar{J}-k_{4}-3 k_{5}+\frac{L \pi_{16}}{12}+\frac{7 \pi_{16}^{2}}{48}
\end{aligned}
$$




$$
\begin{aligned}
& V_{210}=\lambda_{1}\left\{\frac{1}{4} \pi_{16}\left[-\frac{s}{3} \bar{B}_{21}+\bar{B}\left(\frac{5 m^{2}}{3}+\frac{s}{6}\right)-L m^{2}\right]+5 \pi_{16}^{2}\left(\frac{m^{2}}{18}+\frac{s}{864}\right)\right\} \\
& +\lambda_{2} \pi_{16}^{2}\left(\frac{m^{2}}{3}+\frac{s}{144}\right)+B^{\epsilon} \pi_{16}\left(\frac{4 m^{2}}{9}+\frac{s}{72}\right) \\
& +\bar{J}\left(\frac{499 m^{2} \pi_{16}}{864}+\frac{7 \pi_{16} s}{432}-\frac{4 L m^{2}}{9}-\frac{L s}{72}\right) \\
& +k_{1}\left(\frac{17 m^{2}}{288}+\frac{s}{96}\right)-\frac{s}{288} k_{2}-\frac{m^{2}}{12} k_{3}-\frac{m^{2}}{24} k_{4}+k_{5}\left(\frac{m^{2}}{2}-\frac{s}{8}\right) \\
& +\frac{4 L^{2} m^{2}}{9}+\frac{L^{2} s}{144}+\pi_{16} L\left(\frac{5 s}{432}-\frac{m^{2}}{6}\right)+\pi_{16}^{2}\left(\frac{m^{2} \pi^{2}}{54}-\frac{47 m^{2}}{216}-\frac{\pi^{2} s}{864}-\frac{11 s}{1296}\right) \\
& V_{211}=\lambda_{1}\left(\frac{1}{3} \pi_{16} \bar{B}+\frac{\pi_{16}^{2}}{18}\right)+\lambda_{2} \frac{\pi_{16}^{2}}{6}+\frac{1}{3} \pi_{16} B^{\epsilon} \\
& +\bar{J}\left(\frac{4 \pi_{16}}{9}-\frac{L}{3}\right)+\frac{k_{1}}{6}-2 k_{5}+\frac{L^{2}}{6}+\frac{2 L \pi_{16}}{9}+\frac{\pi_{16}^{2}}{27} \\
& V_{212}=\lambda_{1}\left(\frac{1}{3} \pi_{16} \bar{B}_{21}+\frac{\pi_{16}^{2}}{54}\right)+\lambda_{2} \frac{\pi_{16}^{2}}{18}+B^{\epsilon} \pi_{16}\left(\frac{1}{9}-\frac{m^{2}}{9 s}\right) \\
& +\bar{J}\left(\frac{L m^{2}}{9 s}-\frac{L}{9}+\frac{m^{2} \pi_{16}}{54 s}+\frac{5 \pi_{16}}{27}\right)+\frac{k_{1}}{24}+\frac{k_{2}}{72}-\frac{k_{5}}{2}+\frac{k_{8}}{6} \\
& +\frac{L^{2}}{18}\left(\frac{m^{2}}{s}+1\right)+L \pi_{16}\left(\frac{m^{2}}{9 s}+\frac{1}{27}\right)+\pi_{16}^{2}\left(\frac{m^{2} \pi^{2}}{108 s}+\frac{m^{2}}{18 s}-\frac{1}{108}\right) \\
& V_{213}=-\lambda_{1}\left(\frac{1}{6} \pi_{16} \bar{B}+\frac{\pi_{16}^{2}}{36}\right)-\lambda_{2} \frac{\pi_{16}^{2}}{12}-\frac{1}{6} \pi_{16} B^{\epsilon} \\
& +\bar{J}\left(\frac{L}{6}-\frac{2 \pi_{16}}{9}\right)-\frac{k_{1}}{12}+k_{5}-\frac{L^{2}}{12}-\frac{L \pi_{16}}{9}-\frac{\pi_{16}^{2}}{54} \\
& V_{31}=\lambda_{1}\left(\frac{19 m^{2} \pi_{16}^{2}}{144}-\frac{1}{6} L m^{2} \pi_{16}-\frac{\pi_{16}^{2} s}{96}\right)+\frac{1}{12} \lambda_{2} m^{2} \pi_{16}^{2} \\
& -\bar{J}\left(\frac{2 m^{2} \pi_{16}}{9}+\frac{\pi_{16} s}{48}\right)-\frac{1}{12} m^{2} k_{1}-\frac{1}{6} m^{2} k_{3}-3 m^{2} k_{4}-5 m^{2} k_{5} \\
& +\frac{L^{2} m^{2}}{6}-\frac{19 L m^{2} \pi_{16}}{72}+\frac{L \pi_{16} s}{48}+\pi_{16}^{2}\left(\frac{m^{2} \pi^{2}}{72}+\frac{47 m^{2}}{1728}-\frac{s}{384}\right) \\
& V_{32}=\frac{1}{2} \lambda_{1}\left[\pi_{16}\left(s \bar{B}_{31}-s \bar{B}_{21}+\frac{1}{2} m^{2} \bar{B}-\frac{1}{3} L m^{2}\right)+\pi_{16}^{2}\left(\frac{113 m^{2}}{144}-\frac{23 s}{288}\right)\right] \\
& +\lambda_{2} \pi_{16}^{2}\left(\frac{5 m^{2}}{24}-\frac{s}{48}\right)+B^{\epsilon} \pi_{16}\left(\frac{m^{2}}{6}-\frac{s}{24}\right) \\
& +\bar{J}\left(\frac{L s}{24}-\frac{L m^{2}}{6}+\frac{23 m^{2} \pi_{16}}{72}-\frac{3 \pi_{16} s}{32}\right)-\frac{m^{2}}{24} k_{1}-\frac{s}{48} k_{2}+m^{2} k_{4}+\frac{5 m^{2}}{2} k_{5} \\
& +\frac{L^{2} m^{2}}{3}-\frac{L^{2} s}{48}+L \pi_{16}\left(\frac{s}{96}-\frac{65 m^{2}}{144}\right)+\pi_{16}^{2}\left(\frac{m^{2} \pi^{2}}{48}+\frac{745 m^{2}}{3456}-\frac{\pi^{2} s}{144}-\frac{19 s}{768}\right) \\
& V_{33}=\lambda_{1} \frac{\pi_{16}^{2}}{24}+\frac{1}{12} \pi_{16} \bar{J}-\frac{k_{3}}{3}-12 k_{4}-60 k_{5}-20 k_{6}-\frac{L \pi_{16}}{12}-\frac{43 \pi_{16}^{2}}{288}
\end{aligned}
$$




$$
\begin{aligned}
& V_{34}=\lambda_{1}\left(\pi_{16} \bar{B}_{31}-\frac{\pi_{16}^{2}}{96}\right)+\lambda_{2} \frac{\pi_{16}^{2}}{8}+B^{\epsilon} \pi_{16}\left(\frac{1}{4}-\frac{m^{2}}{2 s}\right) \\
& +\bar{J}\left(\frac{L m^{2}}{2 s}-\frac{L}{4}-\frac{5 m^{2} \pi_{16}}{6 s}+\frac{19 \pi_{16}}{48}\right) \\
& +\frac{19 k_{1}}{128}-\frac{3 k_{2}}{128}-\frac{9 k_{4}}{32}+\frac{9 k_{5}}{8}+\frac{5 k_{6}}{2}+\frac{3 k_{9}}{32} \\
& +\frac{L^{2} m^{2}}{4 s}+\frac{L^{2}}{8}+\pi_{16} L\left(\frac{m^{2}}{2 s}+\frac{5}{48}\right)+\pi_{16}^{2}\left(\frac{m^{2} \pi^{2}}{24 s}+\frac{m^{2}}{4 s}+\frac{323}{1152}\right) \\
& V_{35}=\lambda_{1} \frac{\pi_{16}^{2}}{48}+\frac{1}{24} \pi_{16} \bar{J}+3 k_{4}+24 k_{5}+10 k_{6}-\frac{L \pi_{16}}{24}-\frac{41 \pi_{16}^{2}}{576} \\
& V_{36}=\lambda_{1} \frac{\pi_{16}^{2}}{24}-\frac{L \pi_{16}}{12}-\frac{37 \pi_{16}^{2}}{288} \\
& +\bar{J}\left(\frac{\pi_{16}}{12}-\frac{m^{2} \pi_{16}}{9 s}\right)+\frac{k_{1}}{192}-\frac{k_{2}}{192}-\frac{k_{4}}{16}-\frac{31 k_{5}}{4}-5 k_{6}+\frac{k_{9}}{48} \\
& V_{37}=\lambda_{1}\left\{\frac{1}{4} \pi_{16}\left[s \bar{B}_{21}+\bar{B}\left(m^{2}-\frac{1}{2} s\right)-\frac{2}{3} L m^{2}\right]+\pi_{16}^{2}\left(\frac{113 m^{2}}{288}-\frac{23 s}{576}\right)\right\} \\
& +\lambda_{2} \pi_{16}^{2}\left(\frac{5 m^{2}}{24}-\frac{s}{48}\right)+B^{\epsilon} \pi_{16}\left(\frac{m^{2}}{6}-\frac{s}{24}\right) \\
& +\bar{J}\left(\frac{L s}{24}-\frac{L m^{2}}{6}+\frac{23 m^{2} \pi_{16}}{72}-\frac{3 \pi_{16} s}{32}\right)-\frac{m^{2}}{24} k_{1}-\frac{s}{48} k_{2}+m^{2} k_{4}+\frac{5 m^{2}}{2} k_{5} \\
& +\frac{L^{2} m^{2}}{3}-\frac{L^{2} s}{48}-\frac{65 L m^{2} \pi_{16}}{144}+\frac{L \pi_{16} s}{96}+\pi_{16}^{2}\left(\frac{m^{2} \pi^{2}}{48}+\frac{745 m^{2}}{3456}-\frac{\pi^{2} s}{144}-\frac{19 s}{768}\right) \\
& V_{38}=\lambda_{1}\left[\frac{1}{4} \pi_{16}\left(s \bar{B}_{21}-s \bar{B}_{31}-\frac{m^{2}}{2} \bar{B}+\frac{L m^{2}}{3}\right)+\pi_{16}^{2}\left(\frac{23 s}{1152}-\frac{113}{576} m^{2}\right)\right] \\
& +\lambda_{2} \pi_{16}^{2}\left(\frac{s}{96}-\frac{5 m^{2}}{48}\right)+B^{\epsilon} \pi_{16}\left(\frac{s}{48}-\frac{m^{2}}{12}\right) \\
& +\bar{J}\left(\frac{L m^{2}}{12}-\frac{L s}{48}-\frac{23 m^{2} \pi_{16}}{144}+\frac{3 \pi_{16} s}{64}\right)+\frac{m^{2}}{48} k_{1}+\frac{s}{96} k_{2}-\frac{m^{2}}{2} k_{4}-\frac{5 m^{2}}{4} k_{5} \\
& -\frac{L^{2} m^{2}}{6}+\frac{L^{2} s}{96}+\frac{65 L m^{2} \pi_{16}}{288}-\frac{L \pi_{16} s}{192}+\pi_{16}^{2}\left(\frac{\pi^{2} s}{288}+\frac{19 s}{1536}-\frac{m^{2} \pi^{2}}{96}-\frac{745 m^{2}}{6912}\right) \\
& V_{39}=\lambda_{1}\left(\frac{1}{12} L m^{2} \pi_{16}-\frac{19}{288} m^{2} \pi_{16}^{2}+\frac{\pi_{16}^{2} s}{192}\right)-\frac{1}{24} \lambda_{2} m^{2} \pi_{16}^{2} \\
& +\bar{J}\left(\frac{m^{2} \pi_{16}}{9}+\frac{\pi_{16} s}{96}\right)+\frac{1}{24} m^{2} k_{1}+\frac{1}{12} m^{2} k_{3}+\frac{3}{2} m^{2} k_{4}+\frac{5}{2} m^{2} k_{5} \\
& -\frac{L^{2} m^{2}}{12}+\frac{19 L m^{2} \pi_{16}}{144}-\frac{L \pi_{16} s}{96}+\pi_{16}^{2}\left(\frac{s}{768}-\frac{m^{2} \pi^{2}}{144}-\frac{47 m^{2}}{3456}\right) \\
& V_{310}=\lambda_{1}\left[\frac{1}{4} \pi_{16}\left(s \bar{B}_{21}-s \bar{B}_{31}-\frac{1}{2} m^{2} \bar{B}+\frac{1}{3} L m^{2}\right)+\pi_{16}^{2}\left(\frac{23 s}{1152}-\frac{113}{576} m^{2}\right)\right] \\
& +\lambda_{2}\left(\frac{\pi_{16}^{2} s}{96}-\frac{5 m^{2} \pi_{16}^{2}}{48}\right)+B^{\epsilon} \pi_{16}\left(\frac{s}{48}-\frac{m^{2}}{12}\right)
\end{aligned}
$$




$$
\begin{aligned}
& +\bar{J}\left(\frac{L m^{2}}{12}-\frac{L s}{48}-\frac{23 m^{2} \pi_{16}}{144}+\frac{3 \pi_{16} s}{64}\right)+\frac{m^{2}}{48} k_{1}+\frac{s}{96} k_{2}-\frac{m^{2}}{2} k_{4}-\frac{m^{2}}{4} k_{5} \\
& -\frac{L^{2} m^{2}}{6}+\frac{L^{2} s}{96}+\frac{65 L m^{2} \pi_{16}}{288}-\frac{L \pi_{16} s}{192}+\pi_{16}^{2}\left(\frac{\pi^{2} s}{288}+\frac{19 s}{1536}-\frac{m^{2} \pi^{2}}{96}-\frac{745 m^{2}}{6912}\right) \\
& V_{311}=\lambda_{1} \frac{\pi_{16}^{2}}{48}+\frac{1}{24} \pi_{16} \bar{J}+3 k_{4}+24 k_{5}+10 k_{6}-\frac{L \pi_{16}}{24}-\frac{41 \pi_{16}^{2}}{576} \\
& V_{312}=\lambda_{1}\left(\frac{\pi_{16}^{2}}{192}-\frac{1}{2} \pi_{16} \bar{B}_{31}\right)-\lambda_{2} \frac{\pi_{16}^{2}}{16}+B^{\epsilon} \pi_{16}\left(\frac{m^{2}}{4 s}-\frac{1}{8}\right) \\
& +\bar{J}\left(\frac{L}{8}-\frac{L m^{2}}{4 s}+\frac{5 m^{2} \pi_{16}}{12 s}-\frac{19 \pi_{16}}{96}\right)-\frac{19 k_{1}}{256}+\frac{3 k_{2}}{256}+\frac{9 k_{4}}{64}-\frac{9 k_{5}}{16}-\frac{5 k_{6}}{4}-\frac{3 k_{9}}{64} \\
& -\frac{L^{2} m^{2}}{8 s}-\frac{L^{2}}{16}-\frac{L m^{2} \pi_{16}}{4 s}-\frac{5 L \pi_{16}}{96}-\pi_{16}^{2}\left(\frac{m^{2} \pi^{2}}{48 s}+\frac{m^{2}}{8 s}+\frac{323}{2304}\right) \\
& V_{313}=-\lambda_{1} \frac{\pi_{16}^{2}}{96}-\frac{1}{48} \pi_{16} \bar{J}-\frac{3 k_{4}}{2}-12 k_{5}-5 k_{6}+\frac{L \pi_{16}}{48}+\frac{41 \pi_{16}^{2}}{1152} \\
& V_{314}=\lambda_{1}\left(\frac{1}{2} \pi_{16} \bar{B}_{21}-\frac{\pi_{16}^{2}}{144}\right)+\lambda_{2} \frac{\pi_{16}^{2}}{12}+B^{\epsilon} \pi_{16}\left(\frac{1}{6}-\frac{m^{2}}{6 s}\right) \\
& +\bar{J}\left(\frac{L m^{2}}{6 s}-\frac{L}{6}-\frac{5 m^{2} \pi_{16}}{18 s}+\frac{5 \pi_{16}}{24}\right)+\frac{35 k_{1}}{384}-\frac{k_{2}}{128}-\frac{3 k_{4}}{32}+\frac{19 k_{5}}{8}+\frac{5 k_{6}}{2}+\frac{k_{9}}{32} \\
& +\frac{L^{2} m^{2}}{12 s}+\frac{L^{2}}{12}+\frac{L m^{2} \pi_{16}}{6 s}+\frac{L \pi_{16}}{8}+\pi_{16}^{2}\left(\frac{m^{2} \pi^{2}}{72 s}+\frac{m^{2}}{12 s}+\frac{97}{576}\right) \\
& V_{315}=\lambda_{1} \frac{\pi_{16}^{2}}{32}+\frac{1}{16} \pi_{16} \bar{J}-\frac{k_{4}}{2}-9 k_{5}-5 k_{6}-\frac{L \pi_{16}}{16}-\frac{127 \pi_{16}^{2}}{1152} \\
& V_{316}=-\lambda_{1} \frac{\pi_{16}^{2}}{48}+\bar{J}\left(\frac{m^{2} \pi_{16}}{18 s}-\frac{\pi_{16}}{24}\right) \\
& -\frac{k_{1}}{384}+\frac{k_{2}}{384}+\frac{k_{4}}{32}+\frac{31 k_{5}}{8}+\frac{5 k_{6}}{2}-\frac{k_{9}}{96}+\frac{L \pi_{16}}{24}+\frac{37 \pi_{16}^{2}}{576} \\
& V_{317}=\lambda_{1}\left[\frac{1}{12} \pi_{16}\left(s \bar{B}_{21}+m^{2} \bar{B}-\frac{1}{2} s \bar{B}-L m^{2}\right)+\pi_{16}^{2}\left(\frac{31 m^{2}}{144}-\frac{7 s}{432}\right)\right] \\
& +\lambda_{2} \pi_{16}^{2}\left(\frac{m^{2}}{12}-\frac{s}{144}\right)+B^{\epsilon} \pi_{16}\left(\frac{m^{2}}{18}-\frac{s}{72}\right) \\
& +\bar{J}\left(\frac{L s}{72}-\frac{L m^{2}}{18}+\frac{101 m^{2} \pi_{16}}{864}-\frac{\pi_{16} s}{27}\right)-\frac{11}{288} m^{2} k_{1}-\frac{1}{144} s k_{2}-\frac{1}{12} m^{2} k_{3} \\
& -\frac{11}{24} m^{2} k_{4}+k_{5}\left(\frac{7 m^{2}}{4}-\frac{3 s}{8}\right)+k_{6}\left(m^{2}-\frac{s}{4}\right) \\
& +\frac{5 L^{2} m^{2}}{36}-\frac{L^{2} s}{144}-\frac{23 L m^{2} \pi_{16}}{72}+\frac{L \pi_{16} s}{108}+\pi_{16}^{2}\left(\frac{m^{2} \pi^{2}}{108}-\frac{m^{2}}{1728}-\frac{\pi^{2} s}{432}-\frac{173 s}{10368}\right) \\
& V_{318}=\lambda_{1}\left[\frac{1}{6} \pi_{16}\left(s \bar{B}_{21}-s \bar{B}_{31}+m^{2} \bar{B}-\frac{1}{2} L m^{2}\right)+\pi_{16}^{2}\left(\frac{m^{2}}{32}+\frac{19 s}{1728}\right)\right] \\
& +\lambda_{2} \pi_{16}^{2}\left(\frac{m^{2}}{8}+\frac{s}{144}\right)+B^{\epsilon} \pi_{16}\left(\frac{7 m^{2}}{36}+\frac{s}{72}\right)
\end{aligned}
$$




$$
\begin{aligned}
& +\bar{J}\left(-\frac{7 L m^{2}}{36}-\frac{L s}{72}+\frac{199 m^{2} \pi_{16}}{864}+\frac{23 \pi_{16} s}{864}\right) \\
& +k_{1}\left(\frac{7 m^{2}}{144}+\frac{s}{192}\right)+\frac{s}{576} k_{2}+\frac{5 m^{2}}{24} k_{4}+k_{5}\left(\frac{s}{8}-\frac{5 m^{2}}{8}\right)+k_{6}\left(\frac{s}{8}-\frac{m^{2}}{2}\right) \\
& +\frac{11 L^{2} m^{2}}{72}+\frac{L^{2} s}{144}+\frac{11 L m^{2} \pi_{16}}{144}+\frac{L \pi_{16} s}{864}+\pi_{16}^{2}\left(\frac{m^{2} \pi^{2}}{216}-\frac{125 m^{2}}{1152}+\frac{\pi^{2} s}{1728}+\frac{85 s}{20736}\right) \\
& V_{319}=\lambda_{1}\left\{\frac{1}{6} \pi_{16}\left[-s \bar{B}_{21}+\bar{B}\left(\frac{1}{2} s-m^{2}\right)+L m^{2}\right]+\pi_{16}^{2}\left(\frac{25 s}{864}-\frac{35}{144} m^{2}\right)\right\} \\
& +\lambda_{2} \pi_{16}^{2}\left(\frac{s}{72}-\frac{m^{2}}{6}\right)+B^{\epsilon} \pi_{16}\left(\frac{s}{36}-\frac{m^{2}}{9}\right) \\
& +\bar{J}\left(\frac{L m^{2}}{9}-\frac{L s}{36}-\frac{2 m^{2} \pi_{16}}{27}+\frac{29 \pi_{16} s}{432}\right)+\frac{1}{18} m^{2} k_{1}+\frac{1}{72} s k_{2}-\frac{1}{3} m^{2} k_{4}-m^{2} k_{5} \\
& -\frac{5 L^{2} m^{2}}{18}+\frac{L^{2} s}{72}+\frac{19 L m^{2} \pi_{16}}{72}-\frac{5 L \pi_{16} s}{432}+\pi_{16}^{2}\left(\frac{\pi^{2} s}{216}+\frac{169 s}{10368}-\frac{m^{2} \pi^{2}}{54}-\frac{397 m^{2}}{1728}\right) \\
& V_{320}=\lambda_{1}\left[\frac{1}{6} \pi_{16}\left(-s \bar{B}_{21}+s \bar{B}_{31}+\frac{1}{2} m^{2} \bar{B}-\frac{1}{2} L m^{2}\right)+\pi_{16}^{2}\left(\frac{35 m^{2}}{288}-\frac{25 s}{1728}\right)\right] \\
& +\lambda_{2} \pi_{16}^{2}\left(\frac{m^{2}}{12}-\frac{s}{144}\right)+B^{\epsilon} \pi_{16}\left(\frac{m^{2}}{18}-\frac{s}{72}\right) \\
& +\bar{J}\left(\frac{L s}{72}-\frac{L m^{2}}{18}+\frac{m^{2} \pi_{16}}{27}-\frac{29 \pi_{16} s}{864}\right)-\frac{m^{2}}{36} k_{1}-\frac{s}{144} k_{2}+\frac{m^{2}}{6} k_{4}+\frac{m^{2}}{2} k_{5} \\
& +\frac{5 L^{2} m^{2}}{36}-\frac{L^{2} s}{144}-\frac{19 L m^{2} \pi_{16}}{144}+\frac{5 L \pi_{16} s}{864}+\pi_{16}^{2}\left(\frac{m^{2} \pi^{2}}{108}+\frac{397 m^{2}}{3456}-\frac{\pi^{2} s}{432}-\frac{169 s}{20736}\right) \\
& V_{321}=\lambda_{1} \frac{\pi_{16}^{2}}{24}+\frac{1}{12} \pi_{16} \bar{J}-6 k_{5}-4 k_{6}-\frac{L \pi_{16}}{12}-\frac{43 \pi_{16}^{2}}{288} \\
& V_{322}=\lambda_{1}\left(\frac{1}{3} \pi_{16} \bar{B}_{31}+\frac{\pi_{16}^{2}}{288}\right)+\lambda_{2} \frac{\pi_{16}^{2}}{24}+B^{\epsilon} \pi_{16}\left(\frac{1}{12}-\frac{m^{2}}{6 s}\right) \\
& +\bar{J}\left(\frac{L m^{2}}{6 s}-\frac{L}{12}+\frac{m^{2} \pi_{16}}{36 s}+\frac{7 \pi_{16}}{48}\right)+\frac{k_{1}}{48}+\frac{k_{2}}{48}+\frac{k_{5}}{2}+\frac{k_{6}}{2}-\frac{3 k_{7}}{2}+\frac{k_{8}}{4} \\
& +\frac{L^{2} m^{2}}{12 s}+\frac{L^{2}}{24}+\frac{L m^{2} \pi_{16}}{6 s}+\frac{L \pi_{16}}{48}+\pi_{16}^{2}\left(\frac{m^{2} \pi^{2}}{72 s}+\frac{m^{2}}{12 s}+\frac{49}{3456}\right) \\
& V_{323}=\lambda_{1} \frac{\pi_{16}^{2}}{72}+\frac{1}{36} \pi_{16} \bar{J}-\frac{3 k_{5}}{2}-k_{6}+k_{7}-\frac{L \pi_{16}}{36}-\frac{43 \pi_{16}^{2}}{864} \\
& V_{324}=\lambda_{1}\left(\frac{1}{6} \pi_{16} \bar{B}+\frac{\pi_{16}^{2}}{144}\right)+\lambda_{2} \frac{\pi_{16}^{2}}{12}+\frac{1}{6} \pi_{16} B^{\epsilon} \\
& +\bar{J}\left(\frac{13 \pi_{16}}{72}-\frac{L}{6}\right)+\frac{k_{1}}{12}+2 k_{5}+2 k_{6}+\frac{L^{2}}{12}+\frac{11 L \pi_{16}}{72}+\frac{161 \pi_{16}^{2}}{1728} \\
& V_{325}=-\lambda_{1} \frac{\pi_{16}^{2}}{48}-\frac{1}{24} \pi_{16} \bar{J}+3 k_{5}+2 k_{6}+\frac{L \pi_{16}}{24}+\frac{43 \pi_{16}^{2}}{576} \\
& V_{326}=-\lambda_{1}\left(\frac{1}{3} \pi_{16} \bar{B}_{21}+\frac{\pi_{16}^{2}}{216}\right)-\lambda_{2} \frac{\pi_{16}^{2}}{18}+B^{\epsilon} \pi_{16}\left(\frac{m^{2}}{9 s}-\frac{1}{9}\right)
\end{aligned}
$$




$$
\begin{aligned}
& +\bar{J}\left(\frac{L}{9}-\frac{L m^{2}}{9 s}-\frac{m^{2} \pi_{16}}{54 s}-\frac{17 \pi_{16}}{108}\right)-\frac{k_{1}}{24}-\frac{k_{2}}{72}-k_{5}-k_{6}+k_{7}-\frac{k_{8}}{6} \\
& -\frac{L^{2} m^{2}}{18 s}-\frac{L^{2}}{18}-\frac{L m^{2} \pi_{16}}{9 s}-\pi_{16}^{2}\left(\frac{m^{2} \pi^{2}}{108 s}+\frac{m^{2}}{18 s}+\frac{35}{864}\right)
\end{aligned}
$$

Where the $\bar{J}$ and $k_{i}$ function are defined as

$$
\begin{aligned}
\sigma & =\sqrt{1-\frac{4}{s}} \\
h & =\frac{1}{\sigma} \ln \frac{\sigma-1}{\sigma+1} \\
\bar{J} & =\pi_{16}\left(\sigma^{2} h+2\right) \\
k_{1} & =\pi_{16}^{2} \sigma^{2} h^{2} \\
k_{2} & =\pi_{16}^{2}\left(\sigma^{4} h^{2}-4\right) \\
k_{3} & =\frac{1}{\left(16 \pi^{2}\right)^{2}}\left[\frac{\sigma^{2}}{s} h^{3}+\pi^{2} \frac{1}{s} h-\frac{\pi^{2}}{2}\right] \\
k_{4} & =\frac{1}{s \sigma^{2}}\left[\frac{1}{2} k_{1}+\frac{1}{3} k_{3}+\pi_{16} \bar{J}+\frac{\pi_{16}^{2}}{12}\left(\pi^{2}-6\right) s\right] \cdot \\
k_{5} & =\frac{1}{s \sigma^{2}}\left[k_{4}-\frac{1}{12} k_{1}-\frac{\pi_{16}}{12} \bar{J}+\pi_{16}^{2}\left(\frac{5}{6}-\frac{\pi^{2}}{9}\right)\right]+\frac{\pi_{16}^{2}}{12}\left(\frac{5}{2}-\frac{1}{3} p i^{2}\right) \\
k_{6} & =\frac{1}{s \sigma^{2}}\left[5 k_{5}+\frac{1}{12} k_{1}+\frac{\pi_{16}}{18} \bar{J}+\frac{\pi_{16}^{2}}{6}\left(\pi^{2}-\frac{49}{6}\right)\right]+\frac{1}{24} \pi_{16}^{2}\left(\pi^{2}-\frac{49}{6}\right) \\
k_{7} & =\frac{1}{s}\left(k_{5}+\frac{1}{2} k_{4}+\frac{1}{24} k_{1}+\frac{5}{24} \pi_{16} \bar{J}\right)+\frac{1}{72} \pi_{16}^{2} \\
k_{8} & =\frac{1}{s}\left(k_{4}+\frac{7}{12} k_{1}+\frac{25}{36} \pi_{16} \bar{J}\right)+\pi_{16}^{2}\left(\frac{47}{216}+\frac{1}{36} \pi^{2}\right) \\
k_{9} & =\frac{1}{s}\left(k_{3}-\frac{5}{2} k_{1}\right)-\pi_{16}^{2}\left(2+\frac{\pi^{2}}{12}\right) \\
\bar{s} &
\end{aligned}
$$

The $\bar{J}$ and $k_{i}$ vanish at $s=0$ and are well behaved for $s \rightarrow \infty$. They have discontinuities in the derivative at threshold but there no poles there. The functions $k_{i}$ are constructed using the arguments and methods of [35].

All the $k_{i}$ above show up at intermediate stages of the calulations but in the final result $k_{5}(s), \ldots, k_{9}(s)$ always appear multiplied by powers of $s$ and can thus be removed.

Finally, to get the scattering lengths we need to expand these functions around $t, u=0$ and $s=4$. The expansion using $s=4\left(1+q^{2}\right)$ around $s=4$ reads up to order $q^{4}$.

$$
\begin{aligned}
\bar{J}(s) & =\pi_{16}\left(2-2 q^{2}+\frac{4}{3} q^{4}\right) \\
k_{1}(s) & =\pi_{16}^{2}\left(-\pi^{2}+4 q^{2}-\frac{4}{3} q^{4}\right) \\
k_{2}(s) & =\pi_{16}^{2}\left(-4-\pi^{2} q^{2}+\left(4+\pi^{2}\right) q^{4}\right) \\
k_{3}(s) & =\pi_{16}^{2}\left(\frac{1}{2} \pi^{2}-\left(2+\frac{2}{3} \pi^{2}\right) q^{2}+\left(2+\frac{8}{15} \pi^{2}\right) q^{4}\right)
\end{aligned}
$$




$$
k_{4}(s)=\pi_{16}^{2}\left(-\frac{2}{3}+\frac{1}{36} \pi^{2}+\left(\frac{1}{3}+\frac{2}{45} \pi^{2}\right) q^{2}-\left(\frac{1}{3}+\frac{4}{105} \pi^{2}\right) q^{4}\right) .
$$

The expansion around $t=0$ up to order $t^{2}$ are

$$
\begin{aligned}
& \bar{J}(t)=\pi_{16}\left(\frac{1}{6} t+\frac{1}{60} t^{2}\right) \\
& k_{1}(t)=\pi_{16}^{2}\left(-t-\frac{1}{12} t^{2}\right) \\
& k_{2}(t)=\pi_{16}^{2}\left(-\frac{2}{3} t-\frac{7}{180} t^{2}\right) \\
& k_{3}(t)=\pi_{16}^{2}\left(\left(-\frac{1}{2}+\frac{1}{12} \pi^{2}\right) t+\left(-\frac{1}{8}+\frac{1}{60} \pi^{2}\right) t^{2}\right) \\
& k_{4}(t)=\pi_{16}^{2}\left(\left(\frac{1}{4}-\frac{1}{36} \pi^{2}\right) t+\left(\frac{19}{240}-\frac{1}{120} \pi^{2}\right) t^{2}\right) .
\end{aligned}
$$

\section{References}

[1] J. Bijnens and J. Lu, JHEP 0911 (2009) 116 [arXiv:0910.5424 [hep-ph]].

[2] M. E. Peskin, Nucl. Phys. B 175 (1980) 197.

[3] J. Preskill, Nucl. Phys. B 177, 21 (1981).

[4] S. Dimopoulos, Nucl. Phys. B 168 (1980) 69.

[5] F. Sannino, Acta Phys. Polon. B 40 (2009) 3533 [arXiv:0911.0931 [hep-ph]].

[6] C. T. Hill and E. H. Simmons, Phys. Rept. 381 (2003) 235 [Erratum-ibid. 390 (2004) 553] arXiv:hep-ph/0203079.

[7] S. Catterall, F. Sannino, Phys. Rev. D76 (2007) 034504. arXiv:0705.1664 [heplat]]; T. Appelquist et al., Phys. Rev. Lett. 104 (2010) 071601 [arXiv:0910.2224 [hep-ph]]; A. Deuzeman, M. P. Lombardo and E. Pallante, Phys. Lett. B 670 (2008) 41 arXiv:0804.2905 [hep-lat]]; L. Del Debbio, B. Lucini, A. Patella, C. Pica and A. Rago, Phys. Rev. D 82 (2010) 014510 arXiv:1004.3206 [heplat]]; T. DeGrand, Y. Shamir and B. Svetitsky, Phys. Rev. D 79 (2009) 034501 arXiv:0812.1427 [hep-lat]]; S. Catterall, J. Giedt, F. Sannino and J. Schneible, JHEP 0811 (2008) 009 [arXiv:0807.0792 [hep-lat]].

[8] M. Luscher, Commun. Math. Phys. 105, 153 (1986).

[9] J. B. Kogut, M. A. Stephanov, D. Toublan, J. J. M. Verbaarschot and A. Zhitnitsky, Nucl. Phys. B 582 (2000) 477 arXiv:hep-ph/0001171.

[10] Y. I. Kogan, M. A. Shifman and M. I. Vysotsky, Sov. J. Nucl. Phys. 42 (1985) 318 [Yad. Fiz. 42 (1985) 504].

[11] H. Leutwyler and A. V. Smilga, Phys. Rev. D 46 (1992) 5607.

[12] A. V. Smilga and J. J. M. Verbaarschot, Phys. Rev. D 51 (1995) 829 arXiv:hep-th/9404031. 
[13] J. Gasser and H. Leutwyler, Nucl. Phys. B 250 (1985) 465.

[14] J. Gasser and H. Leutwyler, Phys. Lett. B 184 (1987) 83.

[15] K. Splittorff, D. Toublan and J. J. M. Verbaarschot, Nucl. Phys. B 620 (2002) 290 arXiv:hep-ph/0108040.

[16] J. Bijnens, G. Colangelo and G. Ecker, JHEP 9902 (1999) 020 arXiv:hep-ph/9902437.

[17] J. Bijnens, G. Colangelo and G. Ecker, Annals Phys. 280 (2000) 100 arXiv:hep-ph/9907333.

[18] S. Weinberg, Phys. Rev. Lett. 17 (1966) 616.

[19] J. Bijnens, G. Colangelo, G. Ecker, J. Gasser and M. E. Sainio, Phys. Lett. B 374, 210 (1996) arXiv:hep-ph/9511397.

[20] J. Bijnens, G. Colangelo, G. Ecker, J. Gasser and M. E. Sainio, Nucl. Phys. B 508 (1997) 263 [Erratum-ibid. B 517 (1998) 639] [arXiv:hep-ph/9707291].

[21] S. R. Coleman, J. Wess and B. Zumino, Phys. Rev. 177 (1969) 2239; C. G. . Callan, S. R. Coleman, J. Wess and B. Zumino, Phys. Rev. 177 (1969) 2247.

[22] J. Gasser and H. Leutwyler, Annals Phys. 158 (1984) 142.

[23] G. F. Chew; S. Mandelstam, Phys. Rev. 119 (1960) 467.

[24] D. E. Neville, Phys. Rev. 132 (1963) 844.

[25] K. Nakamura et al. (Particle Data Group), J. Phys. G 37 (2010) 075021.

[26] G. Girardi, A. Sciarrino and P. Sorba, J. Phys. A 15 (1982) 1119.

[27] G. Girardi, A. Sciarrino and P. Sorba, J. Phys. A 16 (1983) 2609.

[28] J. Stern, H. Sazdjian and N. H. Fuchs, Phys. Rev. D 47 (1993) 3814 arXiv:hep-ph/9301244.

[29] J. Gasser and M. E. Sainio, Eur. Phys. J. C 6 (1999) 297 arXiv:hep-ph/9803251].

[30] http://www.thep.lu.se/ bijnens/chpt.html

[31] G. Amoros, J. Bijnens and P. Talavera, Nucl. Phys. B 602 (2001) 87 arXiv:hep-ph/0101127.

[32] J. A. M. Vermaseren, arXiv:math-ph/0010025.

[33] G. Passarino and M. J. G. Veltman, Nucl. Phys. B 160 (1979) 151.

[34] J. Bijnens and P. Talavera, JHEP 0203 (2002) 046 arXiv:hep-ph/0203049.

[35] M. Knecht, B. Moussallam, J. Stern and N. H. Fuchs, Nucl. Phys. B 457 (1995) 513 arXiv:hep-ph/9507319. 